\title{
Senegal: Second Review Under the Policy Support Instrument, Request for a Twelve- Month Arrangement Under the Exogenous Shocks Facility, and Request for Waivers and Modification of Assessment Criteria-Staff Report; Staff Statement; Press Release on the Executive Board Discussion; and Statement by the Executive Director for Senegal
}

In the context of the second review under the Policy Support Instrument, request for a twelve-month arrangement under the Exogenous Shocks Facility, and request for waivers and modification of assessment criteria with Senegal, the following documents have been released and are included in this package:

- $\quad$ The staff report for the Second Review Under the Policy Support Instrument, Request for a Twelve-Month Arrangement Under the Exogenous Shocks Facility, and Request for Waivers and Modification of Assessment Criteria, prepared by a staff team of the IMF, following discussions that ended on November 7, 2008, with officials of Senegal on economic developments and policies. Based on information available at the time of these discussions, the staff report was completed on December 5, 2008. The views expressed in the staff report are those of the staff team and do not necessarily reflect the views of the Executive Board of the IMF.

- A staff statement of December 19, 2008 updating information on recent developments.

- $\quad$ A Press Release summarizing the views of the Executive Board as expressed during its December 19, 2008 discussion of the staff report that completed the review and requests.

- $\quad$ A statement by the Executive Director for Senegal.

The documents listed below have been or will be separately released.

Letter of Intent sent to the IMF by the authorities of Senegal*

Memorandum of Economic and Financial Policies by the authorities of Senegal*

Technical Memorandum of Understanding*

*Also included in the Staff Report

The policy of publication of staff reports and other documents allows for the deletion of market-sensitive information.

Copies of this report are available to the public from International Monetary Fund • Publication Services

$70019^{\text {th }}$ Street, N.W. $\bullet$ Washington, D.C. 20431

Telephone: (202) 623-7430 • Telefax: (202) 623-7201

E-mail: publications@imf.org Internet: http://www.imf.org

Price: $\$ 18.00$ a copy

\section{International Monetary Fund Washington, D.C.}





\title{
INTERNATIONAL MONETARY FUND
}

\section{SENEGAL \\ Second Review Under the Policy Support Instrument, Request for a Twelve-Month Arrangement Under the Exogenous Shocks Facility, and Request for Waivers and Modification of Assessment Criteria}

\author{
Prepared by the African Department \\ (In consultation with other departments)
}

Approved by Benedicte Vibe Christensen and Anthony R. Boote

December 5, 2008

Second PSI Review. Staff recommends completion of the review, notwithstanding significant concerns about budgetary slippages, the associated need for three waivers on assessment criteria (AC), and the related misreporting (see separate report). This is based on the authorities' strong corrective actions to address the slippages, significant program commitments to address the identified weaknesses in the PFM systems and fiscal reporting, a reiteration of the program's medium-term fiscal objectives, strong structural performance under the program, and the urgent need to reinvigorate economic activity and shore up donor support and FDI inflows in the current fragile international environment.

Access to ESF. The authorities request a one-year high-access-component ESF arrangement (30 percent of quota, equivalent to SDR 48.54 million) to help finance the balance of payments impact of higher oil and food prices, to be disbursed in two equal tranches at the completion of the second and third PSI reviews (the latter expected in June 2009). ESF program targets will parallel the PSI's.

Program Discussions. They were held in Dakar during October 28-November 7, 2008, preceded by a fact-finding staff visit during September 16-22, 2008 and discussions in Washington prior to the Annual Meetings. The team comprised Messrs. Mueller (head), Lakwijk, Gitton (all AFR), Ms. Mitra (SPR), Ms. Sancak (FAD), Mr. Michel (PFM expert, FAD), and Mr. Segura-Ubiergo (resident representative). The team met with Finance Minister Diop, Budget Minister Sow, BCEAO National Director Sene, other senior government officials, and representatives of development partners, the private sector, and banks. The mission partially overlapped with a Fund TA mission on PFM arranged to provide a diagnostic of the budgetary slippages and recommend remedies. 


\section{Contents}

Executive Summary

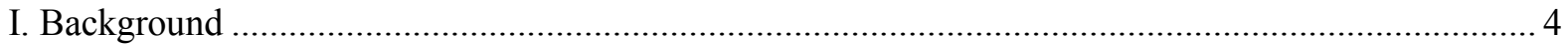

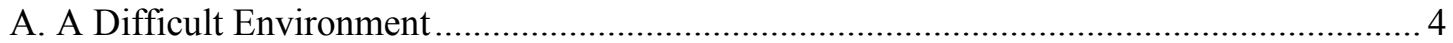

B. Program Performance and Impact of Budgetary Slippages ............................................. 4

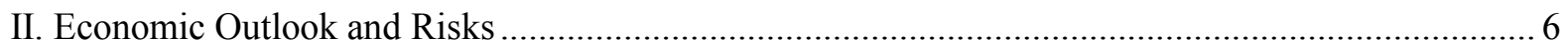

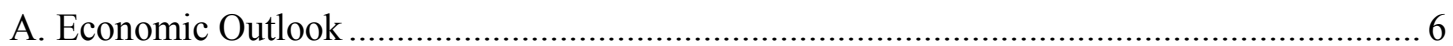

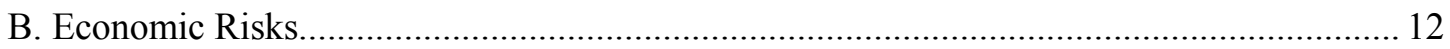

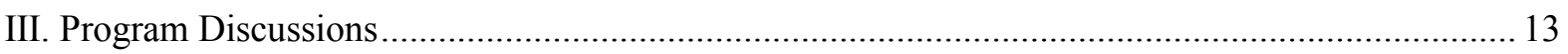

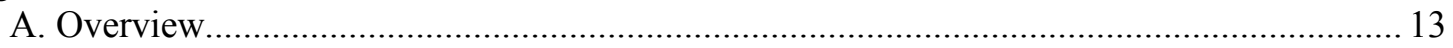

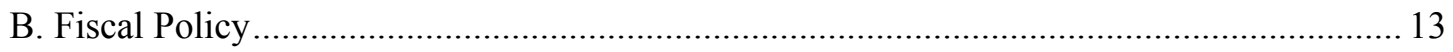

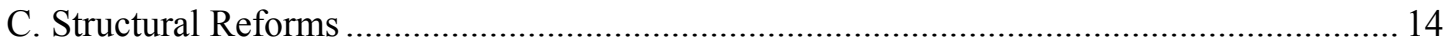

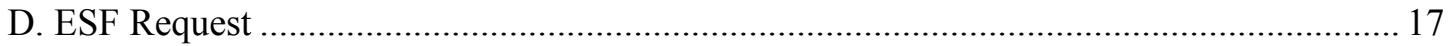

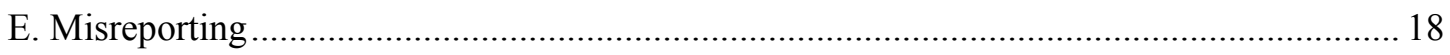

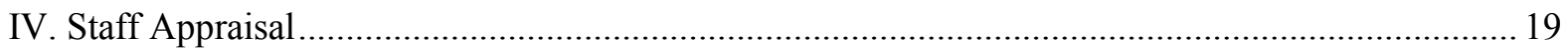

Twelve-Month Arrangement Under the Exogenous Shocks Facility ................................................ 32

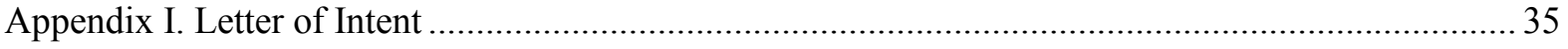

Attachment I. Memorandum on Economic and Financial Policies ................................................... 37

Attachment II. Technical Memorandum of Understanding........................................................... 51

Boxes

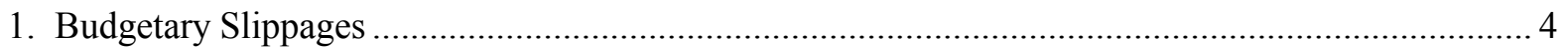

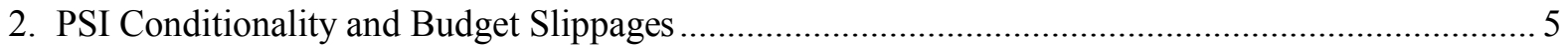

3. Possible Effects of the Global Economic and Financial Crisis ....................................................... 12

4. Conclusions and Recommendations of TA Mission on PFM ................................................... 15

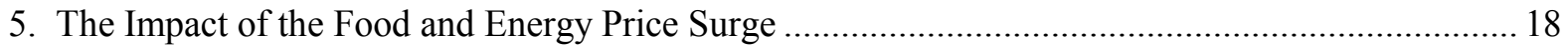

Figures

1. Recent Macroeconomic Developments, 2002-08 …............................................................ 7

2. Senegal, WAEMU, and SSA: Macroeconomic Developments and Outlook, 2004-09 ................... 8

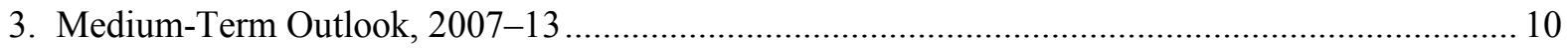

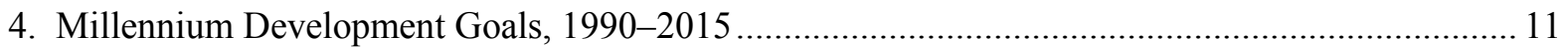

Tables

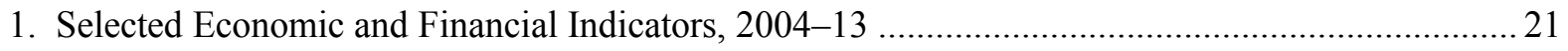

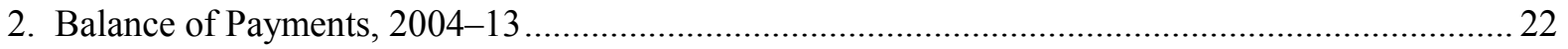

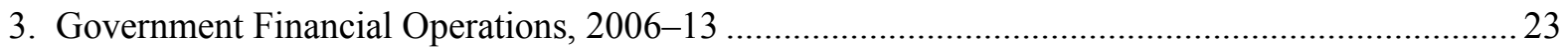

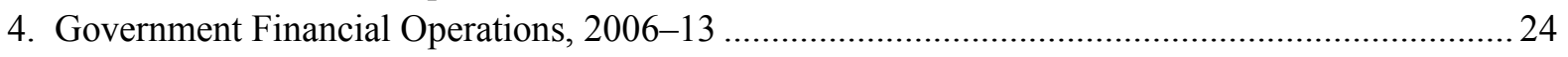

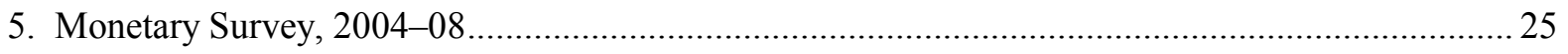

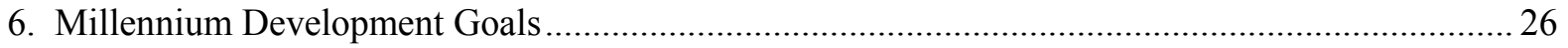

7. Financial Soundness Indicators for the Banking Sector, 2003-08 ............................................ 27

8. Quantitative Assessment Criteria and Indicative Targets, 2007-08 ............................................. 28

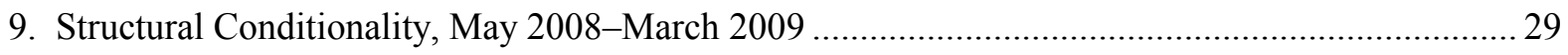

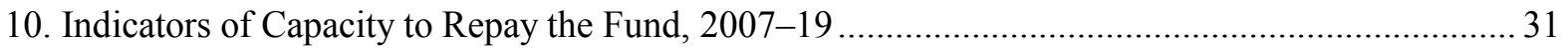




\section{EXECUTIVE SUMMARY}

The second PSI review is taking place in a difficult context arising from exogenous and domestic factors. Rising food and energy prices presented a shock to the balance of payments of 51/4 percent of GDP in 2008. These difficulties have been compounded by significant policy slippages in the form of government payment delays to the private sectoraccumulated over 2006-08 - which are impacting the real economy. Finally, the global financial crisis may affect Senegal in the next few months.

Program performance under the PSI has been mixed. On the positive side, all structural conditionality for the second PSI review has been met, including a new electricity tariff structure. By contrast, significant fiscal slippages (equivalent to 5 percent of GDP) have been uncovered. Program conditionality has been instrumental in identifying a large stock of unpaid government bills and extrabudgetary spending. The payment delays are due in part to the past cost of food and energy subsidies. The unpaid bills and their resolution require three waivers, which staff recommends be granted in light of the strong corrective actions, which will also help remedy the fiscal reporting deficiencies that gave rise to misreporting.

The authorities intend to address with urgency the payment delays and root causes of the budgetary slippages, as also announced by the President. Payment delays are expected to be eliminated by mid-2009. The authorities have already sharply cut spending in the context of a supplementary budget for 2008 and under the 2009 budget law to be adopted in mid-December. They have also committed to reinforcing their PFM systems, based on the diagnostics and recommendations of Fund TA. Among other things, the authorities will improve budget preparation procedures, modify key budget execution practices that have given rise to the slippages, and strengthen fiscal reporting. Three prior actions that achieve immediate and significant improvements in the budgetary area demonstrate the authorities' commitment. The program also includes a strong reiteration by the authorities to pursue a prudent fiscal policy stance, aimed at preserving debt sustainability.

To help address the balance-of-payments impact of the food and energy price shock, the authorities request access to the ESF. They have already taken steps to contain imports, including by eliminating costly and untargeted subsidies for food and energy products, broadening agricultural production, and embarking on energy sector reform. The remaining subsidy on butane gas will be eliminated by mid-2009.

On balance, staff supports completion of the second PSI review and the ESF request. Staff considers that the economy should be in a better position to weather the impact of the global financial downturn with the strong remedial actions under the program to address the impact of both the food and fuel price shocks and the budgetary slippages. 


\section{BACKGROUND}

\section{A. A Difficult Environment}

\section{Senegal's economy has been buffeted by dual shocks at a time when the world} economic and financial crisis is further raising its vulnerability. First, the sharp run-up in international food and energy prices during 2006-08 has increased the import bill. Second, partially related to the high budgetary costs of untargeted subsidies the government had introduced as a first response to the price increases, the government has accumulated sizable payment delays to the private sector, which are affecting the economy. Although the global economic and financial crisis has so far had limited impact on Senegal, its effects may be felt in the months ahead through various channels (see Box 3 below). This provides a difficult context for completing the second PSI review while presenting a basis for access to the ESF.

\section{B. Program Performance and Impact of Budgetary Slippages}

\section{Senegal's PSI program is being tested by the uncovering of major budgetary} slippages (Box 1). They comprise a large stock of unpaid bills vis-à-vis the private sector and large extrabudgetary spending. They have caused some donors to suspend their budget support and, including as a result of corrective actions, also led to the nonobservance of three $\mathrm{ACs}$ (on domestic arrears, the budgetary float, and nonconcessional external borrowing).

\section{Box 1. Budgetary Slippages}

Since early 2006 (when the last PRGF arrangement expired), government payment delays to the private sector and extrabudgetary spending have occurred. This was largely caused by energy and food subsidies introduced in 2006 and significantly expanded in 2007, accounting for a total of 7 percent of GDP during 2006-08. Other spending, especially under the ambitious investment program, was not reigned in until well into 2008.

This reflected unrealistic budgets and a weak budget execution. Unsettled payment orders were carried over to the next year for payment, which in turn crowded out resources for spending committed in that year-a snowballing effect. Heavy reliance on Treasury advances (avances de trésorerie) to pay for unscheduled spending also crowded out regular payments. ${ }^{1}$ Moreover, several line ministries and other public entities contracted goods and services without budgetary appropriations. Ultimately, the stock of unpaid bills became unmanageable.

After a lengthy stocktaking exercise, the authorities identified the following slippages:

- A stock of payment delays to the private sector within the expenditure chain estimated at CFAF 225 billion (3 $3 / 4$ percent of GDP) at end-October 2008, of which CFAF 50 billion owed through agencies.

- Extrabudgetary spending — without budgetary appropriations - of CFAF 74 billion (11/4 percent of GDP) identified by the Ministry of Finance's audit inspectorate (IGF), of which CFAF 11 billion by line ministries and CFAF 63 billion by agencies and universities.

\footnotetext{
${ }^{1}$ A Treasury advance is a ministerial instruction to pay that bypasses regular checks and balances.
} 
3. Program conditionality was instrumental in uncovering the extent of budgetary slippages (Box 2). In August 2008, preliminary IGF audit results became available and the end-June budgetary float under the broadened definition was reported with a level more than three times above the relevant AC (Table 8). The Budget Minister was dismissed.

\section{Box 2: PSI Conditionality and Budget Slippages}

Tight monitoring under the PSI, including through conditionality adjustments at the first review, brought the budgetary slippages to the surface:

- The program included one quantitative AC on domestic arrears (the narrow WAEMU definition) and a broader one on the budgetary float, out of a total of five quantitative ACs. The budgetary float, originally defined as payment orders not yet paid, was broadened at the first review to verified bills not yet paid.

- Coverage of the expenditure tracking software SIGFIP was extended to payments beginning in 2008 (a structural AC) to allow monitoring of the entire expenditure chain and determination of all unpaid spending.

- At the first review, staff was able to identify a stock of payment delays of about 2 percent of GDP and the possibility of extrabudgetary spending (see IMF Country Report No. 08/209). Since it was suspected that the problem could be larger, the IGF audit was included as a structural benchmark for end-September 2008.

\section{The authorities rapidly designed and are implementing an action plan to}

eliminate the unpaid bills (see text table). They sharply curtailed expenditure in a supplementary budget, eliminated costly and untargeted subsidies — consistent with Fund policy advice from the Article IV discussions earlier this year-and raised rapid financing from France, albeit at nonconcessional terms (see below). The supplementary budget also regularized all Treasury advances. The authorities will shortly launch technical audits for the extrabudgetary spending as a basis for a decision on whether such spending should be honored. In addition, they have committed to the sale of government assets to help complete the settling of all budgetary slippages in the first half of 2009. 
Senegal: Key Measures under Authorities' Action Plan

\begin{tabular}{|c|c|}
\hline Action & Effect \\
\hline \multicolumn{2}{|l|}{ Expenditures } \\
\hline $\begin{array}{l}\text { Reduce by CFAF } 100 \text { billion ( } 1 \frac{3 / 4}{4} \text { percent of GDP) in } \\
2008\end{array}$ & Helps create room to settle payment delays \\
\hline \multicolumn{2}{|l|}{ Subsidies } \\
\hline Regularly adjust electricity prices & $\begin{array}{l}\text { Implements market pricing; helps maintain } \\
\text { financial equilibrium of SENELEC }\end{array}$ \\
\hline Reinstate taxes on food, including rice & Reestablishes market pricing; reduces spending \\
\hline $\begin{array}{l}\text { Eliminate butane gas subsidy from mid-2009 and cap } \\
\text { subsidy before }\end{array}$ & Establishes market pricing; reduces spending \\
\hline Eliminate protective tax on vegetable oil & Pro-poor; lowers retail price by 25 percent \\
\hline Expand school feeding program & $\begin{array}{l}\text { Pro-poor; redirects subsidies (cost of } 0.2 \text { percent } \\
\text { of GDP by 2010) }\end{array}$ \\
\hline \multicolumn{2}{|l|}{ Financing and grants } \\
\hline Obtain financing of around $2-21 / 2$ percent of GDP & Provides financing to help settle payment delays \\
\hline Accelerate donor grants in the pipeline & Eases government liquidity constraints \\
\hline $\begin{array}{l}\text { Sale of government assets (e.g., cell phone license, } \\
\text { hotel) }\end{array}$ & $\begin{array}{l}\text { Provides financing in } 2009 \text {, possibly needed for } \\
\text { full settlement of payment delays }\end{array}$ \\
\hline \multicolumn{2}{|l|}{ Public expenditure reform } \\
\hline $\begin{array}{l}\text { Regularize treasury advances in a supplementary } \\
\text { budget }\end{array}$ & Improves transparency and accountability \\
\hline Audit extrabudgetary expenditures & $\begin{array}{l}\text { Allows assessment of which spending is to be } \\
\text { accepted or rejected }\end{array}$ \\
\hline
\end{tabular}

5. All structural conditionality for the second PSI review was met (Table 9):

- A new electricity tariff structure was introduced with reduced rates for low-income consumers (structural assessment criterion), coupled with a 17 percent rise in rates;

- An implementation decree for the special economic zone (DISEZ) was adopted (structural assessment criterion);

- A two-year program of issuing government securities was prepared (structural benchmark), although $1 \frac{1}{2}$ months late;

- The IGF audit of payment delays was completed (structural benchmark);

- Tax collections were consolidated in one department (structural benchmark); and

- An administrative order was issued to strengthen investment planning and evaluation (structural assessment criterion).

\section{ECONOMIC OUTLOOK AND RISKS}

\section{A. Economic Outlook}

6. The budgetary slippages have begun to affect the economy (Figures 1 and 2). Activity in certain sectors, including construction and real estate, is slowing, banks' 
Figure 1. Senegal: Recent Macroeconomic Developments, 2002-08

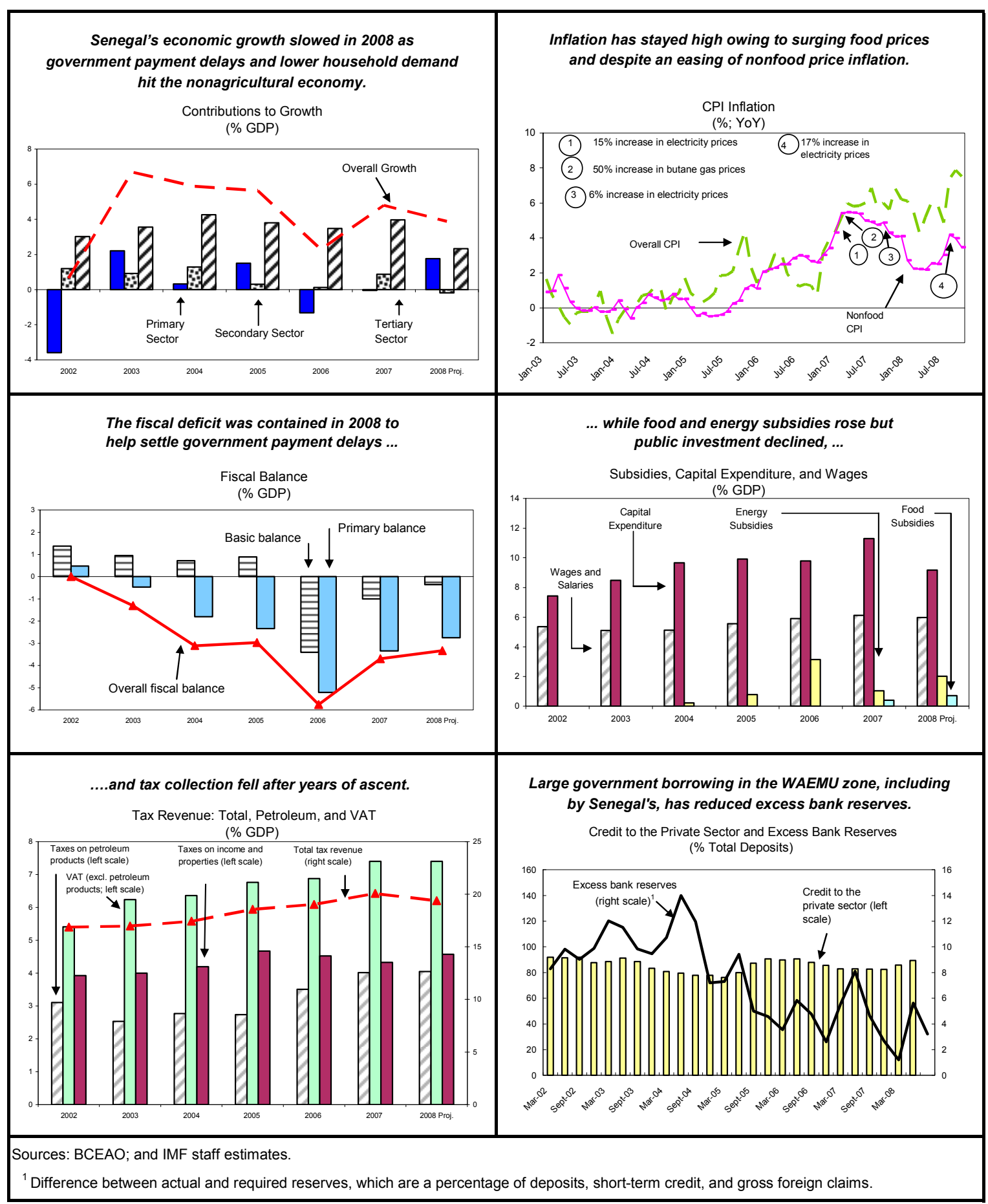


Figure 2. Senegal, WAEMU, and SSA: Macroeconomic Developments and Outlook, 2004-09

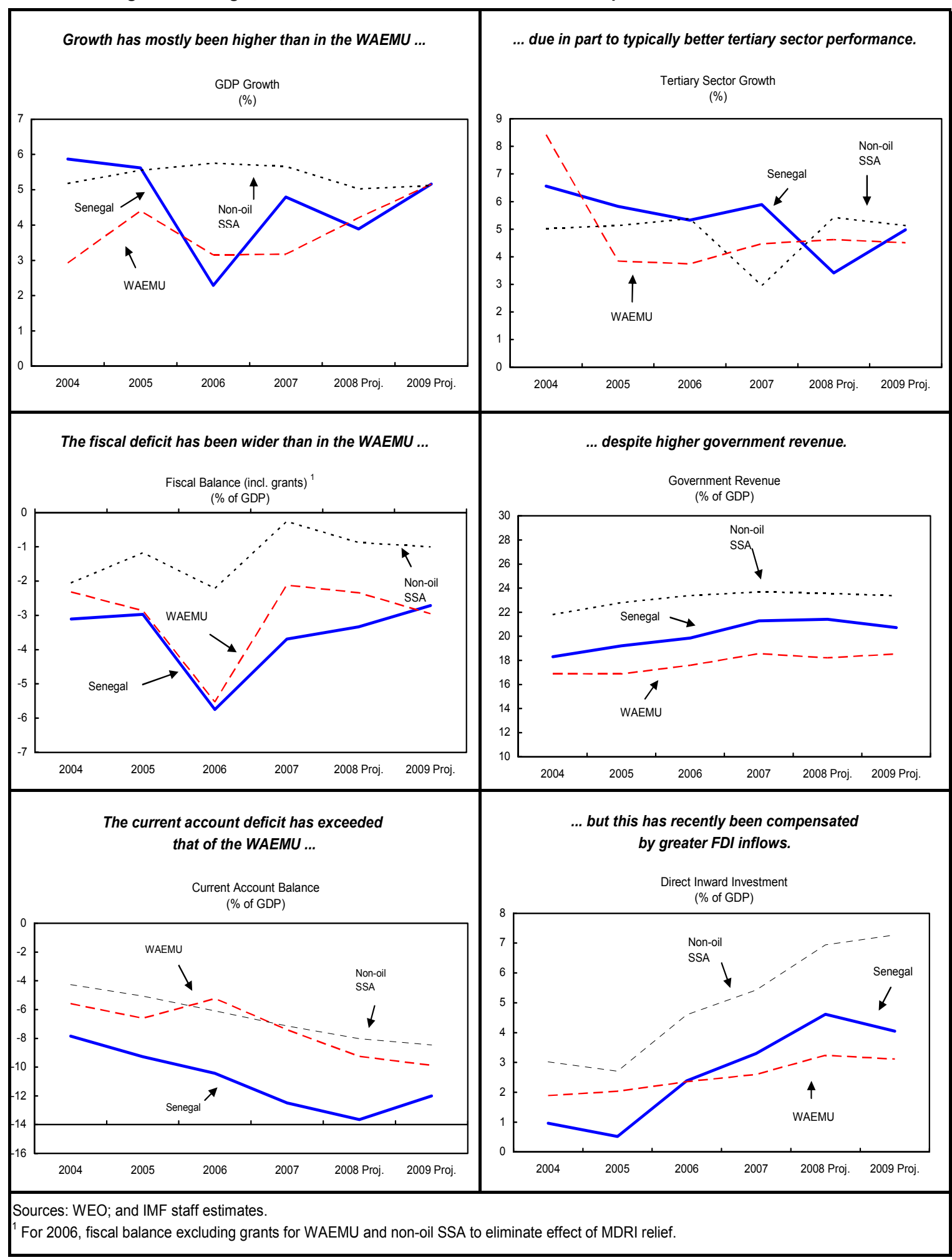


nonperforming loans are on the rise, and tax collections are lagging. ${ }^{1}$ Together with the effect of higher oil and food prices on consumption, this may lower economic growth in 2008 to 3.9 percent.

\section{Senegal's medium-term economic outlook nonetheless remains broadly}

favorable (Figure 3). This hinges on the government quickly normalizing its financial relations with the private sector:

- Economic growth. Supported by the recovery of Industries Chimiques du Sénégal (ICS) ${ }^{2}$ and continued strong FDI inflows, ${ }^{3}$ economic growth is expected to average $5 \frac{1}{2}$ percent during 2009-13, concentrated in construction, telecommunications, and transportation.

- MDGs. Despite some progress, some MDGs will be difficult to achieve (Figure 4, Table 6, and JSAN).

- Inflation. Headline inflation is projected at 6 percent in 2008, reflecting high input prices for food and energy products, with relatively subdued core inflation of 2-3 percent. Inflation is expected to return to historical levels over the medium term.

- Balance of payments and external debt. The current account impact of high food prices will be mitigated by ICS's recovery, but some deterioration is expected over the medium term due to FDI-related imports. FDI inflows,

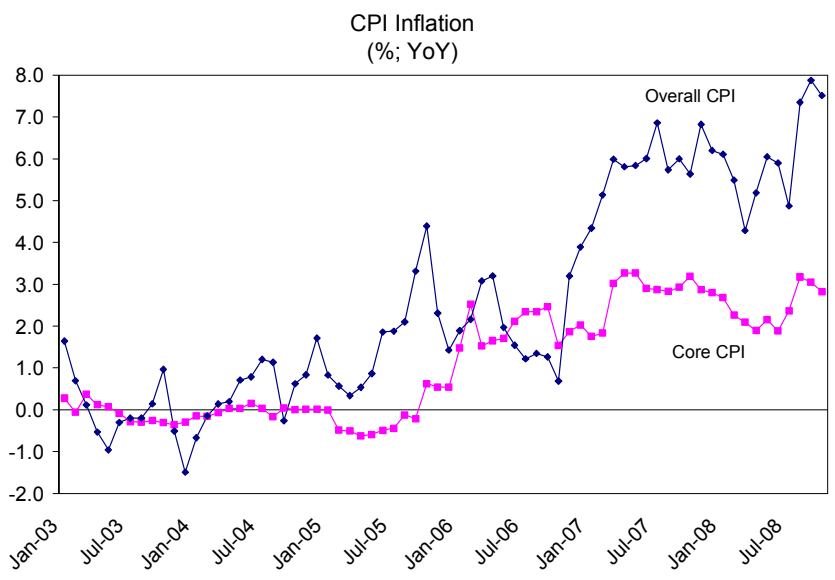
projections of which are so far holding up, should help Senegal maintain its low debt ratios, and the risk of debt distress remains low. ${ }^{4}$ Reserves should hover around $3 \frac{1}{2}$ months of imports.

\footnotetext{
${ }^{1}$ Additional reasons for reduced revenues in 2008 are lower petroleum imports and a change in their composition.

2 ICS, Senegal's largest company which produces phosphoric acid, was successfully rehabilitated in March 2008 and is projected to impact 2009 growth. The foreign majority owner has injected US\$100 million.

${ }^{3}$ Box 4 in IMF Country Report No. 08/209 provides background on the projected rise in FDI.

${ }^{4}$ See the most recent DSA (IMF Country Report No. 08/209, Supplement 1).
} 
Figure 3. Senegal: Medium-Term Outlook, 2007-13

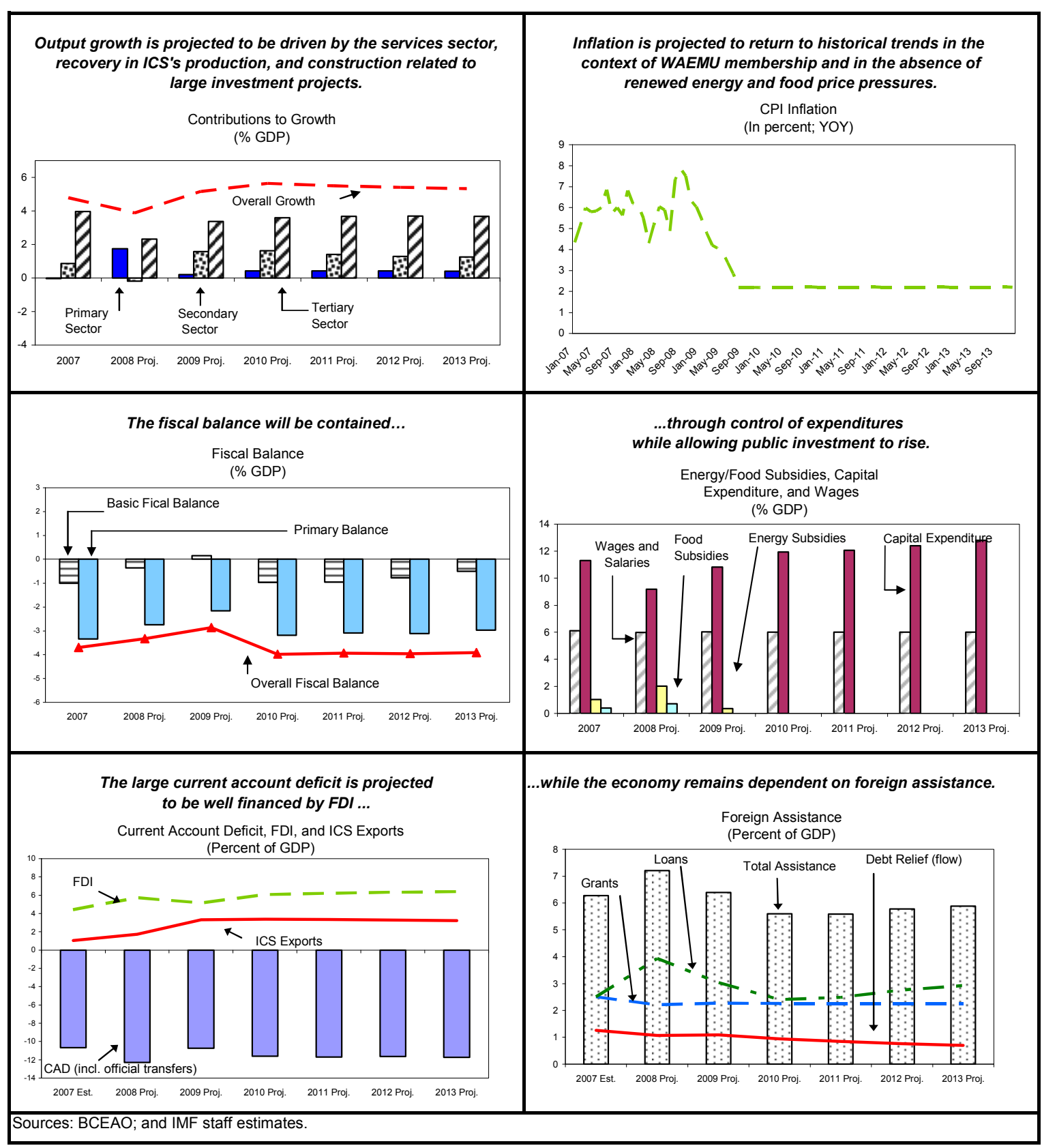


Figure 4. Senegal: Millennium Development Goals, 1990-2015

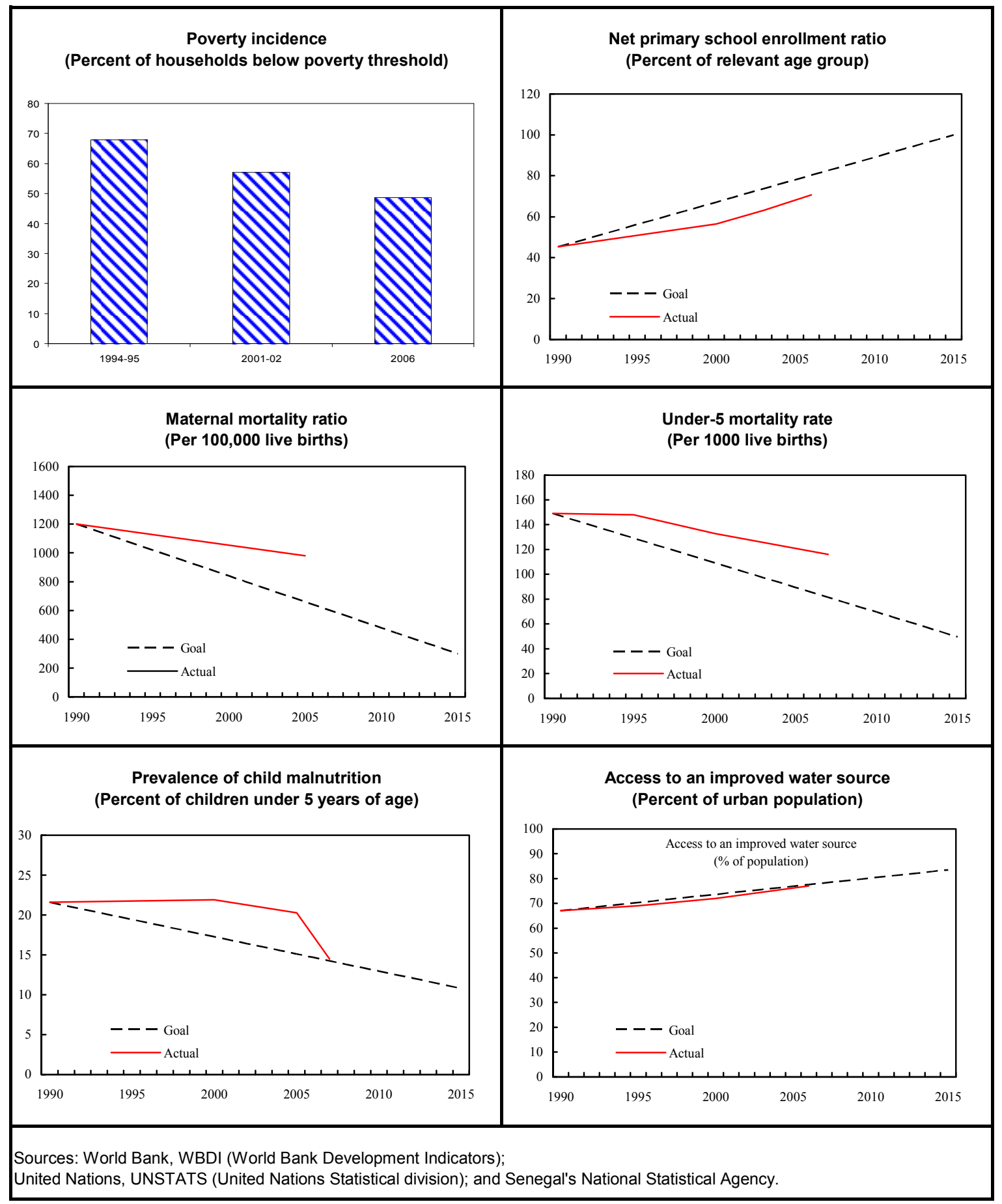




\section{B. Economic Risks}

8. Should payment delays not be settled speedily, Senegal may enter a pronounced economic downturn. This may be exacerbated by the global economic and financial crisis (Box 3).

\section{Box 3. Possible Effects of the Global Economic and Financial Crisis}

The international crisis comes at a time when economic activity has been affected by domestic policy slippages. It may be transmitted through the following channels, which the authorities indicated they would monitor closely and proactively:

Aid flows: Budget constraints in donor countries resulting from domestic bailout packages could reduce aid (which recently amounted to 2 percent of GDP annually for Senegal). Senegal may be at a disadvantage in the future allocation of aid, as some budget aid is currently suspended.

Remittances: A pronounced economic downturn in Europe would likely dampen remittances (running at 8 percent of GDP per year) and affect economic activity, such as in construction.

FDI: A delay or cancellation of FDI (projected to rise from $1 / 2$ percent of GDP in 2005 to 5 percent of GDP in 2010 - see Box 4 in IMF Country Report No. 08/209) could affect financing of the current account and lower Senegal's growth and employment prospects.

Financing flows: While Senegal relies mostly on concessional borrowing, large investment projects developed under Public-Private Partnerships (e.g., new airport, toll highway) may be delayed should financing conditions remain adverse, and fresh financing could dry up. The private sector could experience a drying up of trade finance.

Exports: Phosphoric acid exports should rise in 2009-10 due to the recovery of ICS, but other exports - especially tourism, processed fish products, and groundnut products - could suffer from a decline in demand in international markets.

Financial sector: The authorities are confident that banks in Senegal may be less affected as they are generally independent subsidiaries with their own capital base and limited credit lines from parent banks abroad. Existing capital controls also make large-scale withdrawals of funds difficult. However, the global economic crisis could lead to deteriorating domestic loan quality.

9. Other risks also need to be carefully monitored. These include: (i) exogenous shocks, such as unpredictable price developments in commodities markets and adverse weather and pest conditions affecting agriculture; and (ii) the political and economic costs of cleaning up the budgetary slippages, which could lead to backtracking in implementing the economic and structural program. 


\section{Program Discussions}

\section{A. Overview}

10. The budgetary slippages and misreporting pose a challenge for completion of the second PSI review. The authorities recognized the need to commit to a strong package that would return Senegal to a prudent fiscal policy, speedily eliminate the payment delays to the private sector, address the extrabudgetary spending, and put in place far-reaching reform measures to restore the integrity of the budget framework and prevent a repeat of the slippages. They recognized the considerable urgency needed for these corrective actions, given that the private sector has been severely impacted and the country risked entering a pronounced economic downturn unless the private sector is repaid.

\section{Senegal's main development partners strongly prefer a continued program} relationship between the Fund and Senegal. They urged the authorities to implement their action plan and agree with staff on strong commitments to bring the program back on track. This would unfreeze budget support from several donors, including France, the Netherlands, and the African Development Bank.

12. In mid-November, the President publicly discussed the authorities' plans. $\mathrm{He}$ emphasized that they center on expenditure control and the mobilization of internal and external resources. The firm goal was to settle all payment delays as soon as possible in 2009. At around the same time, the Minister of Finance provided further details to parliament.

13. In line with the views of the authorities, the program will continue to be based on four pillars: (i) containing the fiscal deficit to underpin macroeconomic stability and safeguard debt sustainability; (ii) improving fiscal governance and transparency so as to enhance policy credibility and sustain external assistance; (iii) encouraging private sector activity by improving the business environment and addressing structural impediments to higher economic growth; and (iv) limiting financial sector vulnerabilities and raising the sector's contribution to the economy. Given the urgency of correcting the fiscal slippages, emphasis is placed in this review on the first two pillars.

\section{B. Fiscal Policy}

14. The medium-term fiscal deficit target of 4 percent of GDP remains valid. This deficit level would keep debt sustainable and contribute to domestic stability within the context of the regional

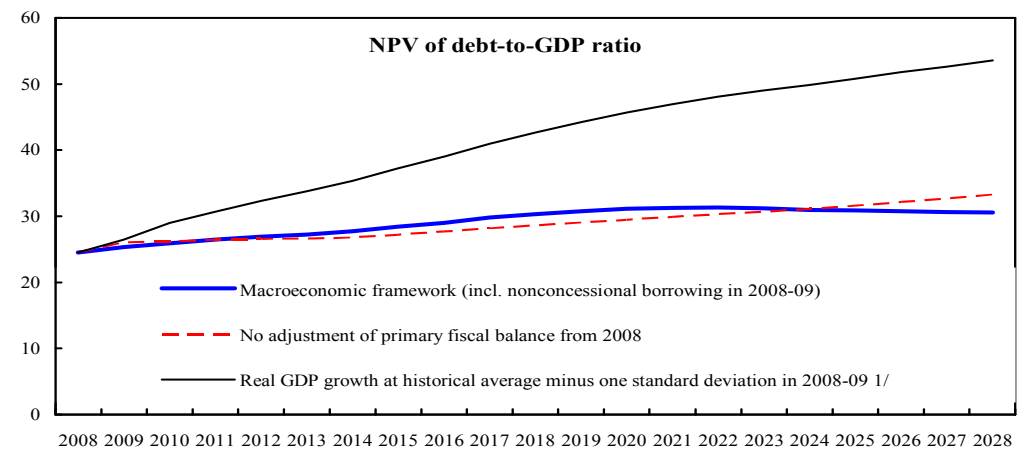

$1 /$ This is the most extreme stress test 
monetary union. However, the availability of financing will have to be carefully considered in the period ahead, and lower deficit targets may be needed if financing cannot be obtained.

\section{The authorities are tightening fiscal policy in $\mathbf{2 0 0 8}$ and 2009 to help settle all} payment delays to the private sector. The mid-November supplementary budget for 2008 included expenditure cuts of around 3 percent of GDP—of which half reflected spending reductions implemented under an administrative order earlier in the year (a first-review prior action). The 2009 budget expected to be passed by parliament by December 15, 2008 (prior action) is in line with the macroeconomic framework developed with the staff-a first for Senegal. ${ }^{56}$ Notwithstanding the tighter budgetary envelope, the authorities committed to safeguarding priority spending.

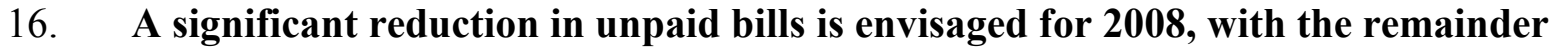
scheduled for the first half of 2009. The repayment schedule depends on available financing, with some possibly disbursed only after the completion of the second PSI review. This requires raising the AC on the budgetary float for end-December 2008 from CFAF 30 billion to CFAF 92 billion. To make meaningful progress in 2008 with eliminating payment delays and reinvigorate economic activity, the authorities requested rapid financial assistance from France, on nonconcessional terms. ${ }^{7}$ This became necessary as other donors had suspended their budget support, the WAEMU market lacks sufficient liquidity, and borrowing in global markets was unattractive. ${ }^{8}$

\section{Structural Reforms}

\section{Structural conditionality focuses on preventing recurrence of the fiscal}

slippages. The key objective is to forcefully address their root causes and strengthen PFM systems. Commitments are also included to make progress in implementing the authorities'

\footnotetext{
${ }^{5}$ Current spending in annual budget laws has generally matched the macroeconomic framework agreed with staff, but the investment budget always exceeded program projections. This may have weakened budget discipline, as line ministries could bypass expenditure cuts undertaken through administrative orders.

${ }^{6}$ In violation of existing procedures, the authorities issued 2007 payment orders as late as November 2008. This led to an upward revision of the 2007 fiscal deficit to 3.7 percent of GDP and misreporting on the basic fiscal balance (see separate misreporting report).

${ }^{7}$ The loan is for $€ 125$ million (CFAF 82 billion), with a maturity of 5 years and an interest rate of 6.2 percent, with 70 percent disbursed in 2008 and the remainder at the time of the third PSI review.

${ }^{8}$ The borrowing spread over comparable US government bonds would have exceeded 10 percentage points.
} 
Accelerated Growth Strategy and the WAEMU FSAP to help the private sector play its intended role as engine of growth and employment.

\section{Conditionality reflects the recommendations of the PFM TA mission. This}

mission was arranged at short notice to urgently help find a way out of the budgetary problems (Box 4). Some improvements require immediate implementation and became prior actions; their completion would also illustrate the authorities' commitment to, and ownership of, the program. Overall, in the staff's view, structural conditionality in the fiscal area-if properly implemented - is sufficiently strong to warrant granting the three requested waivers and correct the fiscal reporting deficiencies that have given rise to misreporting.

\section{Box 4. Conclusions and Recommendations of TA mission on PFM}

The TA mission stressed the generally satisfactory budgetary legal and regulatory framework, the good quality of Ministry of Finance staff, and the well-developed capacity for macroeconomic and budgetary analysis. The expenditure tracking software SIGFIP is also an important asset, although further refinements are warranted.

However, the budgetary framework is weakly applied in three areas:

- Preparation of the annual budget lacks a meaningful top-down constraint, a calendar that is conducive for setting budgetary priorities, and flexibility for unforeseen spending.

- Budget execution is complex, with multiple modifying procedures (budget transfers, credits, and Treasury advances), inefficient expenditure controls, and an absence of account closure; for example, 2007 payment orders were still issued in November 2008.

- Accounting, with substantial delays in the submission of accounts and budget closure laws to the audit court, and no reconciliation of budgetary and Treasury systems.

The authorities broadly agreed with the TA mission's diagnostics and expressed interest in implementing the key recommendations and incorporating them in the budget reform matrix that has been developed with donors. Program conditionality was selected in each of the three areas, focusing on achieving quick results in restoring budgetary discipline.

\section{Objective: Enhance budget preparation and ensure consistency of budget with macroeconomic conditions and available financing.}

- Formulate realistic budgets. The authorities will issue a decree to fix a budget preparation calendar, effective with the 2010 budget, strengthen analysis of the budget's macroeconomic underpinnings, determine the authorities' budgetary priorities and a top-down constraint early in the process, and include 
early consultations with parliament (structural AC). ${ }^{9}$ In addition, the 2009 budget should be consistent with the macroeconomic framework elaborated with staff (prior action). The envisaged adoption of two supplementary budgets around mid-year and near year-end will contribute to enhanced budget realism.

\section{Objective: Strengthen budget execution, improve cash-flow planning, and facilitate fiscal policy monitoring.}

- Eliminate Treasury advances. A decree has eliminated Treasury advances (completed prior action). A continuous zero ceiling for spending MEFP \ 15, 21 undertaken outside the normal and streamlined expenditure procedure (including Treasury advances) will apply from the time of the second PSI review (quantitative $\mathrm{AC}){ }^{10}$

- Limit spending carryover to next fiscal year. A decree will limit carryovers to 5 percent of total spending (structural AC). The government also committed to adjusting spending in the following year to maintain consistency with the macro framework.

- Ensure timely closure of fiscal year. A recent administrative order has set out the deadlines for expenditure commitments, verifications, payment orders, and payments. The 2008 accounts will be closed by end-April 2009 through freezing the SIGFIP system and publishing accounts on the internet (structural AC).

- Reconcile budgetary and accounting data. The authorities reconciled above- and belowthe-line fiscal data from various budgetary and accounting systems and issued a manual outlining the reconciliation procedure (completed prior action). This was necessary to restore confidence in the fiscal data and allow fiscal policy monitoring, including under the program. ${ }^{11}$

\section{Objective: Address extrabudgetary spending.}

- Conduct technical audits of extrabudgetary spending. Based on terms of reference to be agreed with staff, the authorities will contract an external audit to assess all

\footnotetext{
${ }^{9}$ MEFP Table 2 lists structural conditionality, including prior actions.

${ }^{10}$ To keep the number of ACs constant, the existing AC on domestic arrears (WAEMU definition) will be discontinued at the time of the second PSI review, as a broader AC (on the budgetary float) applies and a new AC on Treasury advances is being introduced.

${ }^{11}$ Weekly SIGFIP reports on budget spending have been provided to staff since August.
} 
terms and conditions of the goods and services delivered under past extrabudgetary spending (structural benchmark). This will form the basis for decisions to either regularize such spending in a supplementary budget in 2009 or reject it.

\section{Objective: Reinforce fiscal governance and assess functioning of new procurement framework.}

- Complete and publish first annual report on implementation of procurement framework. The new procurement framework is widely considered a major achievement, but a possible single-tender purchase of a coal power plant by the public electricity company (SENELEC) has raised concerns. The report will assess implementation of the framework (structural benchmark).

\section{Objective: Support fiscal policy stance, shore up macroeconomic} stability, and strengthen fiscal governance.

- Ringfence revenue impact of special economic zone (DISEZ). Memoranda of understanding will identify the respective roles and responsibilities of the entities (zone administrator, revenue authority, and customs authority) involved in the tax administration of the zone (structural benchmark). This measure, set already at the first review, completes a series of structural program conditions related to DISEZ.

\section{Objective: Increase financial intermediation and access to credit.}

- Complete creation of new legal and regulatory framework for microfinance. The sector accounts for one-tenth of credits and, while expanding rapidly, suffers from insufficient supervision and a proliferation of unviable institutions. The new microfinance law (a structural benchmark of the first PSI review) will be made operational by decree (structural benchmark).

\section{ESF Request}

19. The authorities request access to the ESF high-access component, at 30 percent of quota, to help absorb the food and energy price shocks (Box 5). ${ }^{12}$ Senegal's capacity to repay the Fund is good (Table 10$)$. The ESF will cover only a fraction ( $1 / 2$ percent of GDP) of the total exogenous shock.

\footnotetext{
12 The ESF will be a twelve-month arrangement with program targets identical to the PSI's.
} 


\section{Box 5. The Impact of the Food and Energy Price Surge}

Recent global surges of food and fuel prices have weakened Senegal's external position. Senegal imports all of its fuel and most basic staples, including rice and wheat. The price effect is worsening Senegal's 2008 current account by $5 \frac{1 / 4}{4}$ percent of GDP, corresponding to a decline of $1 \frac{1}{2}$ months of reserves.

Taking into account mitigating factors, reserves are expected to fall to $3 \frac{1 / 4}{4}$ months of imports in 2008 (Table 2).

The temporary price subsidies to help shield the population cost 3 percent of GDP in 2008. The recent decision to rescind most subsidies helps reduce budgetary pressures.

\section{ESF access and measures taken by the authorities will}

facilitate countering the shock. The ESF (30 percent of quota equally tranched in two disbursements at the completion of the second and third reviews) will finance food and fuel imports of $1 / 2$ percent of GDP and may catalyze donor support once the current budgetary slippages have been resolved.

20. The authorities are addressing the exogenous shocks as part of their action plan. Consistent with Fund policy advice, ${ }^{13}$ they recently discontinued the rice subsidy, reinstated taxes on core food products, and ended the protective tax on vegetable oil - the latter a longstanding Bank and Fund staff recommendation. This phasing out of subsidies and tax suspensions on food and energy will help contain demand and improve MEFP व 14, 31-33 the balance of payments. Energy sector reform supported by the World Bank and France should diversify energy production, reduce costs, and enhance governance. The butane gas subsidy will be eliminated by mid-2009. Structural policies, including to enhance agricultural production, will be implemented to reduce Senegal's vulnerability to exogenous shocks.

\section{E. Misreporting}

\section{A separate report on} misreporting has been issued. The authorities misreported data related to the basic fiscal balance and the budgetary float for end-2007 under the PSI. ${ }^{14}$ The misreporting partially
Senegal: Misreporting for end-December 2007

\begin{tabular}{lrrr}
\hline & $\begin{array}{r}\text { Adjusted IMF Country } \\
\text { assessment } \\
\text { criteria }\end{array}$ & $\begin{array}{r}\text { Report No. } \\
08 / 209\end{array}$ & $\begin{array}{r}\text { Accounting } \\
\text { Mastem, 2008 }\end{array}$ \\
\hline Floor on the basic fiscal balance & \multicolumn{3}{c}{ (Billions of CFA francs) } \\
Ceiling on the amount of the float & -41 & -39 & -53 \\
& 34 & 33 & 55 \\
\hline
\end{tabular}

${ }^{13}$ See Box 5 in IMF Country Report No. 08/209 and Chapter II in IMF Country Report No. 08/221.

${ }^{14}$ No indications for misreporting were found regarding the previous PRGF. 
reflects budget monitoring and execution deficiencies, which are being addressed under the program. Strong corrective actions are incorporated in the program to prevent a repeat of the misreporting.

\section{Staff Appraisal}

22. The Senegal program supported under the PSI is at a crossroad. Against the backdrop of a difficult international environment, past budgetary slippages need to be cleaned up and prevented going forward. The budgetary slippages that the program helped uncover have undermined the soundness of the private sector and seriously affected economic activity. With the associated misreporting, they have raised concerns about the authorities' credibility and ability to implement their economic program under the PSI. On the other hand, the transparency and cooperation shown in uncovering the slippages, as well as the expeditious design of a strong action plan to eliminate the payment delays and begin the restoration of the integrity of the budget framework, are commendable and represent a solid basis for completing the second review.

\section{A quick resolution of the budgetary slippages is of paramount importance to} shore up economic growth in an unsupportive external environment. The elimination of payment delays to which the authorities are committed under the program should lead to a recovery of private sector activity and have a positive impact on the banking system and tax collections. It should also help reassure donors and shore up investor confidence-which is key in light of the important role of FDI for medium-term growth.

\section{The prudent fiscal policy stance for 2009-10 should help promote domestic} stability and safeguard debt sustainability. The adoption of the 2009 budget consistent with the macroeconomic framework developed with staff is particularly noteworthy. Notwithstanding the tight budgetary environment, the authorities need to preserve budgetary allocations to priority social sectors so as to help bring about a further reduction in poverty and progress toward meeting the MDGs.

\section{Staff welcomes the loan from France to assist Senegal in these exceptionally}

difficult circumstances. Its impact on debt sustainability is negligible, given in particular its one-off character and terms comparable to those in the WAEMU financial market. The limited regional liquidity and the current turmoil in global financial markets left the Senegalese authorities with no alternative to provide urgently needed relief to the ailing private sector. Staff therefore recommend granting a waiver for the nonobservance of the AC on nonconcessional external borrowing due to the contracting of the French loan. Staff would suggest that the authorities reserve the proceeds from asset sales currently being contemplated for early repayment of this loan and the loan for the toll highway, as well as a possibly accelerated settlement of extrabudgetary spending following the technical audit. 
26. The PFM measures included in the program need to be forcefully implemented. They are fundamental to restoring the integrity of the budget system by improving fiscal accounting and monitoring, strengthening budget formulation and execution, and discontinuing practices inconsistent with Senegal's overall satisfactory budgetary legal and regulatory framework and administrative capacity. Staff call on the authorities to take appropriate and immediate decisions following completion of the independent technical audit of past extrabudgetary spending to rapidly and fully normalize financial relations with the private sector. The strong corrective actions in the PFM area, together with the envisaged return to a prudent fiscal policy stance, justify granting waivers for the ACs on domestic arrears and the budgetary float.

27. ESF access should provide important relief for Senegal's balance of payments. The authorities' decision to rescind most of the untargeted subsidies for food and energy products was instrumental in restoring sustainability, as are the reforms in the energy and agricultural sectors. The end to the butane gas subsidy by mid-2009 is also welcome.

28. Staff recommends, on balance, completion of the second PSI review. While the seriousness of the budgetary slippages made it difficult to arrive at this recommendation, the authorities' corrective actions and program commitments are sufficiently strong to warrant it. This strength is especially important given that, in the current difficult global environment, Senegal needs to rapidly reinvigorate economic growth and shore up aid and FDI flows. It is now up to the Senegalese authorities to regain their credibility with the international community. 
Table 1. Senegal: Selected Economic and Financial Indicators, 2004-13

\begin{tabular}{|c|c|c|c|c|c|c|c|c|c|c|}
\hline & 2004 & 2005 & 2006 & 2007 & 2008 & 2009 & 2010 & 2011 & 2012 & 2013 \\
\hline & & & & Est. & \multicolumn{6}{|c|}{ Proj. } \\
\hline & \multirow{2}{*}{\multicolumn{10}{|c|}{ (Annual percentage change) }} \\
\hline National income and prices & & & & & & & & & & \\
\hline GDP at constant prices & 5.9 & 5.6 & 2.3 & 4.8 & 3.9 & 5.2 & 5.6 & 5.5 & 5.4 & 5.3 \\
\hline Of which: nonagriculture GDP & 6.0 & 4.8 & 3.9 & 5.7 & 2.7 & 5.5 & 5.8 & 5.6 & 5.5 & 5.4 \\
\hline GDP deflator & 0.5 & 2.3 & 3.4 & 5.4 & 7.8 & 2.3 & 2.2 & 2.1 & 2.1 & 2.2 \\
\hline \multicolumn{11}{|l|}{ Consumer prices } \\
\hline Annual average & 0.5 & 1.7 & 2.1 & 5.9 & 6.1 & 3.3 & 2.2 & 2.2 & 2.2 & 2.2 \\
\hline End of period & 1.7 & 1.4 & 3.9 & 6.2 & 6.0 & 2.2 & 2.2 & 2.2 & 2.2 & 2.2 \\
\hline \multicolumn{11}{|l|}{ External sector } \\
\hline Exports, f.o.b. (in CFA francs) & 9.2 & 4.4 & 0.1 & -3.9 & 26.1 & 5.6 & 6.7 & 5.7 & 5.7 & 6.0 \\
\hline Imports, f.o.b. (in CFA francs) & 9.8 & 15.6 & 9.0 & 13.1 & 23.3 & 1.0 & 9.6 & 7.7 & 7.7 & 6.7 \\
\hline Export volume & 4.1 & -4.2 & -12.3 & -1.9 & 6.3 & 12.6 & 4.2 & 4.2 & 4.4 & 4.8 \\
\hline Import volume & 2.0 & 4.2 & 5.6 & 7.9 & 7.0 & 8.2 & 7.6 & 6.4 & 6.3 & 6.3 \\
\hline Terms of trade (deterioration (-)) & -2.6 & -2.0 & 10.7 & -6.8 & 2.6 & 0.4 & 0.6 & 0.2 & -0.1 & 0.8 \\
\hline Nominal effective exchange rate & 2.2 & -0.3 & 0.2 & 1.4 & $\cdots$ & $\ldots$ & $\ldots$ & $\ldots$ & $\ldots$ & $\ldots$ \\
\hline \multirow[t]{2}{*}{ Real effective exchange rate } & 0.1 & -1.3 & -0.2 & 4.6 & $\cdots$ & $\cdots$ & $\cdots$ & $\cdots$ & $\cdots$ & $\ldots$ \\
\hline & \multicolumn{10}{|c|}{ (Changes in percent of beginning-of-year broad money, unless otherwise indicated) } \\
\hline \multicolumn{11}{|l|}{ Money and credit } \\
\hline Net domestic assets & 3.1 & 8.6 & 5.0 & 8.6 & 5.7 & 5.0 & 10.3 & 10.0 & 9.4 & 8.7 \\
\hline Domestic credit & 2.5 & 10.4 & 5.8 & 11.5 & 6.0 & 5.3 & 10.7 & 10.4 & 9.7 & 9.0 \\
\hline Credit to the government (net) & -3.1 & -4.1 & 3.0 & 4.9 & -1.1 & -1.7 & 3.3 & 2.8 & 2.2 & 1.5 \\
\hline \multirow[t]{2}{*}{ Credit to the economy (percentage growth) } & 9.2 & 24.5 & 4.2 & 10.5 & 11.3 & 10.9 & 11.4 & 11.6 & 11.8 & 11.8 \\
\hline & \multicolumn{10}{|c|}{ (Percent of GDP, unless otherwise indicated) } \\
\hline \multicolumn{11}{|l|}{ Government financial operations } \\
\hline Revenue & 18.3 & 19.2 & 19.9 & 21.3 & 20.2 & 20.7 & 20.6 & 20.9 & 21.1 & 21.3 \\
\hline Grants & 2.1 & 1.6 & 1.5 & 2.6 & 2.2 & 2.4 & 2.3 & 2.3 & 2.3 & 2.3 \\
\hline Total expenditure and net lending & 23.3 & 24.1 & 27.5 & 27.9 & 25.7 & 25.9 & 26.8 & 27.0 & 27.3 & 27.5 \\
\hline \multicolumn{11}{|l|}{ Overall fiscal surplus (+) or deficit (-) } \\
\hline Payment order basis, excluding grants & -5.2 & -4.6 & -7.3 & -6.3 & -5.5 & -5.1 & -6.2 & -6.1 & -6.2 & -6.2 \\
\hline Payment order basis, including grants & -3.1 & -3.0 & -5.8 & -3.7 & -3.3 & -2.7 & -4.0 & -3.9 & -4.0 & -3.9 \\
\hline Primary fiscal balance $1 /$ & -1.8 & -2.3 & -5.2 & -3.3 & -2.7 & -2.0 & -3.2 & -3.1 & -3.1 & -3.0 \\
\hline Basic fiscal balance 2/ & 0.7 & 0.9 & -3.4 & -1.0 & -0.4 & 0.1 & -0.9 & -0.9 & -0.8 & -0.5 \\
\hline Gross domestic investment & 26.0 & 29.5 & 29.7 & 31.2 & 30.5 & 31.2 & 32.4 & 32.9 & 33.4 & 34.1 \\
\hline Government & 9.7 & 9.9 & 9.8 & 11.3 & 9.2 & 10.8 & 11.9 & 12.1 & 12.5 & 12.9 \\
\hline Nongovernment & 16.3 & 19.6 & 19.9 & 19.9 & 21.3 & 20.4 & 20.5 & 20.8 & 21.0 & 21.2 \\
\hline Gross domestic savings & 13.4 & 14.1 & 12.2 & 10.7 & 9.4 & 12.7 & 13.1 & 13.5 & 13.9 & 14.6 \\
\hline Government & 8.4 & 7.9 & 3.5 & 7.2 & 5.6 & 8.7 & 9.0 & 9.2 & 9.5 & 10.0 \\
\hline Nongovernment & 5.0 & 6.2 & 8.8 & 3.5 & 3.8 & 4.0 & 4.2 & 4.3 & 4.5 & 4.6 \\
\hline Gross national savings & 19.9 & 21.8 & 20.3 & 20.5 & 18.2 & 20.6 & 20.8 & 21.2 & 21.8 & 22.4 \\
\hline \multicolumn{11}{|l|}{ External current account deficit (-) } \\
\hline Including current official transfers & -6.1 & -7.8 & -9.4 & -10.7 & -12.3 & -10.6 & -11.6 & -11.7 & -11.6 & -11.7 \\
\hline Excluding current official transfers & -7.9 & -9.3 & -10.4 & -12.5 & -13.7 & -12.0 & -12.9 & -13.0 & -12.9 & -13.0 \\
\hline Central government domestic debt $3 /$ & 3.5 & 3.3 & 4.3 & 5.6 & 6.9 & 5.9 & 6.9 & 7.8 & 8.3 & 8.5 \\
\hline External public debt (nominal) 3/4/ & 44.0 & 42.4 & 17.8 & 18.1 & 19.6 & 22.0 & 22.1 & 22.3 & 22.9 & 23.6 \\
\hline External public debt service (percent of exports) 4/ & 7.4 & 5.6 & 4.2 & 4.6 & 2.9 & 3.8 & 3.9 & 3.9 & 3.9 & 3.7 \\
\hline External public debt service (percent of government revenue) $4 /$ & 10.9 & 7.9 & 5.4 & 5.1 & 3.6 & 4.6 & 4.7 & 4.5 & 4.4 & 4.1 \\
\hline Gross domestic product (billions of CFA francs) & 4,243 & 4,582 & 4,846 & 5,352 & 5,993 & 6,450 & 6,962 & 7,503 & 8,078 & 8,692 \\
\hline
\end{tabular}

Sources: Senegalese authorities; and Fund staff estimates and projections.

$1 /$ Defined as total revenue and grants minus total expenditure and net lending, excluding interest expenditure.

2/ Defined as total revenue minus total expenditure and net lending, excluding externally financed capital expenditure, on-lending,

and expenditure financed with HIPC Initiative and MDRI assistance.

3/ Debt outstanding at year-end.

4/ After HIPC and MDRI (from 2006) debt relief. 
Table 2. Senegal: Balance of Payments, 2004-13

\begin{tabular}{|c|c|c|c|c|c|c|c|c|c|c|}
\hline & 2004 & 2005 & 2006 & 2007 & 2008 & 2009 & 2010 & 2011 & 2012 & 2013 \\
\hline & & & & Est. & \multicolumn{6}{|c|}{ Proj. } \\
\hline & \multicolumn{10}{|c|}{ (Billions of CFA francs, unless otherwise indicated) } \\
\hline Current account & -257 & -355 & -455 & -571 & -735 & -682 & -808 & -877 & -941 & $-1,017$ \\
\hline Balance on goods & -521 & -691 & -828 & $-1,078$ & $-1,307$ & $-1,274$ & $-1,427$ & $-1,559$ & $-1,704$ & $-1,827$ \\
\hline Exports, f.o.b. & 798 & 832 & 833 & 801 & 1,010 & 1,066 & 1,138 & 1,202 & 1,270 & 1,347 \\
\hline Imports, f.o.b. & $-1,319$ & $-1,524$ & $-1,661$ & $-1,879$ & $-2,316$ & $-2,340$ & $-2,565$ & $-2,761$ & $-2,974$ & $-3,173$ \\
\hline Services and incomes (net) & -78 & -70 & -65 & -62 & -1 & 15 & 6 & 21 & 40 & 27 \\
\hline Credits & 437 & 518 & 512 & 530 & 611 & 651 & 695 & 748 & 805 & 858 \\
\hline Debits & -514 & -587 & -577 & -592 & -612 & -636 & -689 & -727 & -765 & -831 \\
\hline Of which: interest on public debt & -41 & -36 & -36 & -24 & -24 & -34 & -37 & -40 & -40 & -51 \\
\hline Unrequited current transfers (net) & 342 & 405 & 437 & 570 & 572 & 578 & 613 & 661 & 724 & 782 \\
\hline Private (net) & 270 & 341 & 409 & 494 & 494 & 490 & 529 & 570 & 625 & 676 \\
\hline Public (net) & 72 & 65 & 28 & 76 & 78 & 87 & 84 & 91 & 99 & 107 \\
\hline Of which: budgetary grants & 19 & 12 & 9 & 48 & 34 & 39 & 31 & 33 & 36 & 39 \\
\hline Capital and financial account & 281 & 234 & 512 & 622 & 707 & 774 & 808 & 930 & 1,021 & 1,082 \\
\hline Capital account & 74 & 69 & 1,234 & 182 & 107 & 126 & 135 & 145 & 156 & 168 \\
\hline Private capital transfers & 4 & 6 & 7 & 7 & 8 & 9 & 9 & 9 & 10 & 10 \\
\hline Project grants & 70 & 63 & 64 & 86 & 99 & 117 & 126 & 136 & 147 & 158 \\
\hline Debt cancellation and other transfers $1 / 2 /$ & 0 & 0 & 1,163 & 89 & 0 & 0 & 0 & 0 & 0 & 0 \\
\hline Financial account & 207 & 165 & -722 & 440 & 600 & 648 & 673 & 785 & 865 & 914 \\
\hline Direct investment & 34 & 28 & 110 & 171 & 271 & 256 & 341 & 378 & 418 & 455 \\
\hline Portfolio investment & -15 & 14 & -11 & 26 & -3 & 15 & 34 & 36 & 38 & 32 \\
\hline Other investment & 188 & 123 & -821 & 243 & 332 & 377 & 298 & 371 & 410 & 427 \\
\hline Public sector (net) & 32 & 33 & $-1,018$ & 114 & 189 & 227 & 134 & 153 & 191 & 228 \\
\hline Of which: disbursements & 157 & 154 & 147 & 156 & 230 & 286 & 195 & 214 & 256 & 302 \\
\hline program loans & 13 & 21 & 39 & 19 & 97 & 53 & 35 & 38 & 41 & 44 \\
\hline project loans & 144 & 133 & 107 & 138 & 133 & 153 & 175 & 193 & 232 & 274 \\
\hline other & 0 & 0 & 0 & 0 & 0 & 80 & -16 & -16 & -16 & -16 \\
\hline amortization & -127 & -122 & $-1,166$ & -54 & -41 & -59 & -61 & -61 & -66 & -74 \\
\hline Private sector (net) & 129 & 84 & 173 & 200 & 143 & 150 & 164 & 218 & 219 & 199 \\
\hline Errors and omissions & 27 & 6 & 24 & -71 & 0 & 0 & 0 & 0 & 0 & 0 \\
\hline Overall balance & 24 & -122 & 57 & 51 & -28 & 92 & 0 & 53 & 81 & 65 \\
\hline Financing & -24 & 122 & -57 & -51 & 9 & -111 & 0 & -53 & -81 & -65 \\
\hline Net foreign assets (BCEAO) & -126 & -9 & -83 & -75 & -2 & -127 & -11 & -62 & -90 & -73 \\
\hline Net use of Fund resources & -23 & -22 & -66 & 0 & 0 & 0 & 0 & -2 & -3 & -3 \\
\hline Purchases/disbursements & 3 & 3 & 11 & 0 & 0 & 0 & 0 & 0 & 0 & 0 \\
\hline Repurchases/repayments & -26 & -24 & -77 & 0 & 0 & 0 & 0 & -2 & -3 & -3 \\
\hline Other & -102 & 12 & -16 & -75 & -2 & -127 & -11 & -61 & -87 & -70 \\
\hline Deposit money banks & 0 & 26 & -37 & 3 & -10 & -11 & -11 & -12 & -12 & -13 \\
\hline Payments arrears (reduction (-)) & 0 & 0 & 0 & 0 & 0 & 0 & 0 & 0 & 0 & 0 \\
\hline Exceptional financing $2 / 3 /$ & 101 & 105 & 63 & 21 & 21 & 27 & 23 & 21 & 21 & 21 \\
\hline Residual financing gap & 0 & 0 & 0 & 0 & 19 & 19 & 0 & 0 & 0 & 0 \\
\hline \multicolumn{11}{|l|}{ Memorandum items: } \\
\hline Current account balance & & & & & & & & & & \\
\hline Including current official transfers (percent of GDP) & -6.1 & -7.8 & -9.4 & -10.7 & -12.3 & -10.6 & -11.6 & -11.7 & -11.6 & -11.7 \\
\hline Excluding current official transfers (percent of GDP) & -7.9 & -9.3 & -10.4 & -12.5 & -13.7 & -12.0 & -12.9 & -13.0 & -12.9 & -13.0 \\
\hline Gross official reserves (billions of CFA francs) & 668 & 662 & 661 & 735 & 739 & 867 & 879 & 941 & 1030 & 1101 \\
\hline (months of imports of GNFS) & 4.8 & 4.1 & 3.8 & 3.8 & 3.2 & 3.7 & 3.4 & 3.4 & 3.5 & 3.5 \\
\hline Gross domestic product & 4,243 & 4,582 & 4,846 & 5,352 & 5,993 & 6,450 & 6,962 & 7,503 & 8,078 & 8,692 \\
\hline
\end{tabular}

Sources: Central Bank of West African States (BCEAO); and Fund staff estimates and projections.

$1 /$ Includes receipts from sale by the government to a Sudanese operator of a telecom license for US $\$ 200$ million in 2007.

2/ Reflects MDRI stock debt relief in 2006. Debt relief from the Fund is recorded as a capital transfer. Debt relief from the IDA and the AfDF on the amounts

falling due in 2006 is shown as exceptional financing, while debt relief on amounts due in 2007 and beyond is recorded as a capital transfer.

3/ Until 2005, HIPC Initiative flow debt relief granted by the IMF is recorded as a grant, and that granted by the World Bank,

the African Development Bank, Paris Club creditors, and Kuwait is recorded as exceptional financing. 
Table 3. Senegal: Government Financial Operations, 2006-13

\begin{tabular}{|c|c|c|c|c|c|c|c|c|c|c|}
\hline & \multirow[t]{2}{*}{2006} & \multicolumn{2}{|c|}{2007} & \multicolumn{2}{|c|}{2008} & 2009 & 2010 & 2011 & 2012 & 2013 \\
\hline & & Prog. & Est. & Prog. & Proj. & \multicolumn{5}{|c|}{ Proj. } \\
\hline & \multicolumn{10}{|c|}{ (Billions of CFA francs, unless otherwise indicated) } \\
\hline Total revenue and grants & 1,036 & 1,202 & 1,277 & 1,377 & 1,341 & 1,493 & 1,593 & 1,734 & 1,884 & 2,050 \\
\hline Revenue & 963 & 1,082 & 1,139 & 1,254 & 1,209 & 1,337 & 1,436 & 1,565 & 1,702 & 1,854 \\
\hline Tax revenue & 922 & 1,039 & 1,088 & 1,222 & 1,161 & 1,302 & 1,398 & 1,524 & 1,658 & 1,807 \\
\hline Income tax & 219 & 244 & 232 & 254 & 274 & 302 & 327 & 356 & 387 & 420 \\
\hline Taxes on goods and services & 533 & 577 & 628 & 697 & 645 & 742 & 810 & 888 & 976 & 1,074 \\
\hline Taxes on petroleum products & 170 & 217 & 215 & 272 & 242 & 258 & 261 & 280 & 295 & 313 \\
\hline Nontax revenue & 41 & 44 & 51 & 32 & 48 & 35 & 38 & 40 & 44 & 47 \\
\hline Grants & 73 & 120 & 138 & 123 & 132 & 156 & 157 & 169 & 182 & 196 \\
\hline Budgetary & 9 & 41 & 53 & 25 & 34 & 39 & 31 & 33 & 36 & 39 \\
\hline Budgeted development projects & 64 & 79 & 86 & 98 & 99 & 117 & 126 & 136 & 147 & 158 \\
\hline Total expenditure and net lending & 1,331 & 1,452 & 1,491 & 1,667 & 1,541 & 1,668 & 1,868 & 2,025 & 2,204 & 2,388 \\
\hline Current expenditure & 829 & 779 & 881 & 1,007 & 994 & 974 & 1,039 & 1,120 & 1,196 & 1,269 \\
\hline Wages and salaries $1 /$ & 286 & 330 & 327 & 358 & 358 & 389 & 418 & 450 & 485 & 522 \\
\hline Interest due & 42 & 35 & 34 & 43 & 35 & 45 & 56 & 62 & 65 & 78 \\
\hline Of which: external 2/ & 36 & 27 & 24 & 31 & 24 & 34 & 37 & 40 & 40 & 51 \\
\hline Other current expenditure & 500 & 414 & 519 & 606 & 601 & 540 & 565 & 608 & 646 & 669 \\
\hline Transfers and subsidies $3 /$ & 308 & 206 & 287 & 363 & 359 & 270 & 277 & 298 & 313 & 312 \\
\hline Of which: SAR and butane subsidy & 66 & 44 & 55 & 95 & 73 & 23 & 0 & 0 & 0 & 0 \\
\hline Of which : SENELEC & 86 & 0 & 0 & 20 & 48 & 0 & 0 & 0 & 0 & 0 \\
\hline Of which: Food subsidies & 0 & 0 & 21 & 56 & 43 & 0 & 0 & 0 & 0 & 0 \\
\hline Goods and services & 186 & 192 & 217 & 229 & 229 & 256 & 276 & 297 & 321 & 345 \\
\hline HIPC and MDRI current spending & 7 & 16 & 15 & 14 & 13 & 14 & 13 & 13 & 12 & 12 \\
\hline Capital expenditure & 475 & 665 & 605 & 660 & 550 & 698 & 831 & 905 & 1,006 & 1,117 \\
\hline Domestically financed & 337 & 464 & 392 & 429 & 324 & 438 & 540 & 588 & 641 & 699 \\
\hline Of which: Without transfers to PEs & 337 & 399 & 327 & 392 & 287 & 429 & 540 & 588 & 641 & 699 \\
\hline HIPC and MDRI financed & 26 & 81 & 60 & 72 & 66 & 56 & 52 & 51 & 49 & 49 \\
\hline Non HIPC/MDRI financed & 311 & 384 & 331 & 356 & 258 & 382 & 488 & 537 & 592 & 650 \\
\hline Externally financed & 138 & 201 & 213 & 231 & 226 & 260 & 291 & 317 & 366 & 418 \\
\hline Net lending & 27 & 8 & 5 & 0 & -3 & 2 & 0 & 0 & 0 & 0 \\
\hline Of which: On-lending & 33 & 16 & 10 & 0 & 5 & 10 & 0 & 0 & 0 & 0 \\
\hline Selected public sector entities balance 4/ & 16 & 5 & 16 & 0 & 0 & 0 & 0 & 0 & 0 & 0 \\
\hline Primary fiscal balance $5 /$ & -236 & -210 & -163 & -247 & -165 & -130 & -220 & -230 & -254 & -260 \\
\hline Overall fiscal balance (including grants) & -279 & -245 & -198 & -290 & -200 & -175 & -275 & -291 & -319 & -339 \\
\hline Overall fiscal balance (excluding grants) & -352 & -364 & -336 & -413 & -332 & -331 & -432 & -461 & -502 & -535 \\
\hline Basic fiscal balance 6/ & -165 & -56 & -54 & -95 & -21 & 10 & -65 & -69 & -62 & -42 \\
\hline Financing & 279 & 245 & 198 & 290 & 200 & 175 & 275 & 291 & 319 & 339 \\
\hline External financing & 121 & 159 & 131 & 252 & 198 & 260 & 181 & 199 & 236 & 268 \\
\hline Drawings & 146 & 181 & 156 & 211 & 230 & 206 & 211 & 230 & 272 & 318 \\
\hline Program loans & 39 & 43 & 19 & 78 & 97 & 53 & 35 & 38 & 41 & 44 \\
\hline Project loans & 107 & 138 & 138 & 133 & 133 & 153 & 175 & 193 & 232 & 274 \\
\hline Amortization due & -58 & -67 & -54 & -47 & -41 & -59 & -61 & -61 & -66 & -74 \\
\hline Debt relief and HIPC Initiative assistance 7/ & 41 & 32 & 21 & 29 & 21 & 27 & 23 & 21 & 21 & 21 \\
\hline T-bills and bonds issued in WAEMU & -8 & 13 & 8 & 19 & -12 & 7 & 25 & 25 & 25 & 18 \\
\hline Non-concessional loans for infrastructure development & 0 & 0 & 0 & 40 & 0 & 80 & -16 & -16 & -16 & -16 \\
\hline Domestic financing & 158 & 85 & 58 & 38 & 67 & -12 & 94 & 93 & 83 & 71 \\
\hline Banking system & 128 & 112 & 98 & 40 & -21 & -37 & 77 & 74 & 64 & 50 \\
\hline Of which: T-bills and bonds & 23 & 155 & 136 & 52 & -13 & -28 & 86 & 85 & 74 & 56 \\
\hline Nonbank financing $8 /$ & 30 & -27 & -40 & -2 & 88 & 24 & 17 & 18 & 20 & 21 \\
\hline Settlement of previous year's payment delays & 0 & 0 & 0 & 0 & -84 & -92 & 0 & 0 & 0 & 0 \\
\hline Errors and omissions & -1 & 0 & 9 & 0 & 0 & 0 & 0 & 0 & 0 & 0 \\
\hline Financing gap & 0 & 0 & 0 & 0 & 19 & 19 & 0 & 0 & 0 & 0 \\
\hline \multicolumn{11}{|l|}{ Memorandum items: } \\
\hline Budgetary float (program definition) & 58 & 34 & 55 & 30 & 92 & 30 & 30 & 30 & 30 & 30 \\
\hline Airport travel tax earmarked for new airport (RDIA) & 18 & 22 & 36 & 38 & 18 & 19 & 20 & 21 & 22 & 23 \\
\hline HIPC Initiative expenditure 9/ & 14 & 49 & $\ldots$ & 44 & $\ldots$ & 27 & 23 & 21 & 21 & 21 \\
\hline Priority expenditure (in percent of total expenditure) $10 /$ & 31 & $\ldots$ & 32 & $\ldots$ & 36 & 38 & 39 & $\ldots$ & $\ldots$ & $\ldots$ \\
\hline Gross domestic product & 4,846 & 5,247 & 5,352 & 5,856 & 5,993 & 6,450 & 6,962 & 7,503 & 8,078 & 8,692 \\
\hline
\end{tabular}

Sources: Senegalese authorities; and Fund staff estimates and projections.

1/ Excludes project-related wages and salaries, which are included in capital spending, and the salaries of autonomous agencies and health and education contractual workers, which are included in transfers and subsidies.

2/ From 2006, reflects post-MDRI debt service schedule.

$3 /$ Excludes subsidies aimed at sector development policies, which are included in capital spending.

4/ Local governments, autonomous public sector entities (e.g., hospitals, universities), and the civil servants' pension fund (FNR).

$5 /$ Defined as total revenue and grants minus total expenditure and net lending, excluding interest expenditure.

6/ Total revenue minus total expenditure and net lending, excluding externally financed capital expenditure, on-lending, and HIPC/MDRI expenditure.

7/ Until 2005, includes HIPC Initiative debt relief accorded by the IMF, the World Bank, the African Development Bank, and Paris Club Creditors.

8/ Includes receipts from sale of telecom license for $\$ 200$ million in late 2007.

9/ Refers to HIPC-financed capital and other expenditure.

10/ Defined as expenditures on health, education, environment, the judiciary, social development, sewage and rural irrigation. 
Table 4. Senegal: Government Financial Operations, 2006-13

\begin{tabular}{|c|c|c|c|c|c|c|c|c|c|c|}
\hline & \multirow[t]{2}{*}{2006} & \multicolumn{2}{|c|}{2007} & \multicolumn{2}{|c|}{2008} & \multirow[t]{2}{*}{2009} & \multirow[t]{2}{*}{2010} & \multirow{2}{*}{$\begin{array}{l}2011 \\
\text { Proj. }\end{array}$} & \multirow[t]{2}{*}{2012} & \multirow[t]{2}{*}{2013} \\
\hline & & Prog. & Est. & Prog. & Proj. & & & & & \\
\hline & \multicolumn{9}{|c|}{ (Percent of GDP) } & \\
\hline Total revenue and grants & 21.4 & 22.9 & 23.9 & 23.5 & 22.4 & 23.1 & 22.9 & 23.1 & 23.3 & 23.6 \\
\hline Revenue & 19.9 & 20.6 & 21.3 & 21.4 & 20.2 & 20.7 & 20.6 & 20.9 & 21.1 & 21.3 \\
\hline Tax revenue & 19.0 & 19.8 & 20.3 & 20.9 & 19.4 & 20.2 & 20.1 & 20.3 & 20.5 & 20.8 \\
\hline Income tax & 4.5 & 4.7 & 4.3 & 4.3 & 4.6 & 4.7 & 4.7 & 4.7 & 4.8 & 4.8 \\
\hline Taxes on goods and services & 11.0 & 11.0 & 11.7 & 11.9 & 10.8 & 11.5 & 11.6 & 11.8 & 12.1 & 12.4 \\
\hline Taxes on petroleum products & 3.5 & 4.1 & 4.0 & 4.6 & 4.0 & 4.0 & 3.7 & 3.7 & 3.7 & 3.6 \\
\hline Nontax revenue & 0.8 & 0.8 & 1.0 & 0.6 & 0.8 & 0.5 & 0.5 & 0.5 & 0.5 & 0.5 \\
\hline Grants & 1.5 & 2.3 & 2.6 & 2.1 & 2.2 & 2.4 & 2.3 & 2.3 & 2.3 & 2.3 \\
\hline Total expenditure and net lending & 27.5 & 27.7 & 27.9 & 28.5 & 25.7 & 25.9 & 26.8 & 27.0 & 27.3 & 27.5 \\
\hline Current expenditure & 17.1 & 14.8 & 16.5 & 17.2 & 16.6 & 15.1 & 14.9 & 14.9 & 14.8 & 14.6 \\
\hline Wages and salaries & 5.9 & 6.3 & 6.1 & 6.1 & 6.0 & 6.0 & 6.0 & 6.0 & 6.0 & 6.0 \\
\hline Interest payments $1 /$ & 0.9 & 0.7 & 0.6 & 0.7 & 0.6 & 0.7 & 0.8 & 0.8 & 0.8 & 0.9 \\
\hline Other current expenditure & 10.3 & 7.9 & 9.7 & 10.4 & 10.0 & 8.4 & 8.1 & 8.1 & 8.0 & 7.7 \\
\hline Of which: Goods and services & 3.8 & 3.7 & 4.1 & 3.9 & 3.8 & 4.0 & 4.0 & 4.0 & 4.0 & 4.0 \\
\hline Of which: Transfers and subsidies & 6.3 & 3.9 & 5.4 & 6.2 & 6.0 & 4.2 & 4.0 & 4.0 & 3.9 & 3.6 \\
\hline Of which: Energy and food subsidies & 3.1 & 0.8 & 1.4 & 2.9 & 2.7 & 0.4 & 0.0 & 0.0 & 0.0 & 0.0 \\
\hline HIPC and MDRI current spending & 0.1 & 0.3 & 0.3 & 0.2 & 0.2 & 0.2 & 0.2 & 0.2 & 0.2 & 0.1 \\
\hline Capital expenditure & 9.8 & 12.7 & 11.3 & 11.3 & 9.2 & 10.8 & 11.9 & 12.1 & 12.5 & 12.9 \\
\hline Domestically financed & 7.0 & 8.8 & 7.3 & 7.3 & 5.4 & 6.8 & 7.8 & 7.8 & 7.9 & 8.0 \\
\hline Of which: Without transfers to PEs & 7.0 & 7.6 & 6.1 & 6.7 & 4.8 & 6.7 & 7.8 & 7.8 & 7.9 & 8.0 \\
\hline Externally financed & 2.8 & 3.8 & 4.0 & 3.9 & 3.8 & 4.0 & 4.2 & 4.2 & 4.5 & 4.8 \\
\hline Net lending & 0.6 & 0.2 & 0.1 & 0.0 & 0.0 & 0.0 & 0.0 & 0.0 & 0.0 & 0.0 \\
\hline Selected public sector entities balance $2 /$ & 0.3 & 0.1 & 0.3 & 0.0 & 0.0 & 0.0 & 0.0 & 0.0 & 0.0 & 0.0 \\
\hline Primary fiscal balance $3 /$ & -5.2 & -4.1 & -3.3 & -4.2 & -2.7 & -2.0 & -3.2 & -3.1 & -3.1 & -3.0 \\
\hline \multicolumn{11}{|l|}{ Overall fiscal balance } \\
\hline Payment order basis, excluding grants & -7.3 & -6.9 & -6.3 & -7.1 & -5.5 & -5.1 & -6.2 & -6.1 & -6.2 & -6.2 \\
\hline Payment order basis, including grants & -5.8 & -4.7 & -3.7 & -4.9 & -3.3 & -2.7 & -4.0 & -3.9 & -4.0 & -3.9 \\
\hline Basic fiscal balance 4/ & -3.4 & -1.1 & -1.0 & -1.6 & -0.4 & 0.1 & -0.9 & -0.9 & -0.8 & -0.5 \\
\hline Financing & 5.8 & 4.7 & 3.7 & 4.9 & 3.3 & 2.7 & 4.0 & 3.9 & 4.0 & 3.9 \\
\hline External financing & 2.5 & 3.0 & 2.4 & 4.3 & 3.3 & 4.0 & 2.6 & 2.7 & 2.9 & 3.1 \\
\hline Domestic financing & 3.3 & 1.6 & 1.1 & 0.6 & 1.1 & -0.2 & 1.3 & 1.2 & 1.0 & 0.8 \\
\hline Settlement of payment delays & 0.0 & 0.0 & 0.0 & 0.0 & -1.4 & -1.4 & 0.0 & 0.0 & 0.0 & 0.0 \\
\hline Errors and omissions & 0.0 & 0.0 & 0.2 & 0.0 & 0.0 & 0.0 & 0.0 & 0.0 & 0.0 & 0.0 \\
\hline Financing gap & 0.0 & 0.0 & 0.0 & 0.0 & 0.3 & 0.3 & 0.0 & 0.0 & 0.0 & 0.0 \\
\hline Memorandum items: & \multicolumn{9}{|c|}{ (Percent of GDP, unless otherwise indicated) } & \\
\hline Airport travel tax earmarked for new airport (RDIA) & 0.4 & 0.4 & 0.7 & 0.6 & 0.3 & 0.3 & 0.3 & 0.3 & 0.3 & 0.3 \\
\hline Priority expenditure $5 /$ & 8.6 & & 9.0 & & 9.2 & 9.9 & 10.5 & & & \\
\hline Wages and salaries (percent of fiscal revenue) & 29.7 & 30.5 & 28.7 & 28.5 & 29.6 & 29.1 & 29.1 & 28.8 & 28.5 & 28.1 \\
\hline
\end{tabular}

Sources: Senegalese authorities; and Fund staff estimates and projections.

1/ From 2006, reflects post-MDRI debt service schedule.

2/ Local governments, autonomous public sector entities (e.g. hospitals, universities), and the civil servants' pension fund (FNR).

$3 /$ Defined as total revenue and grants minus total expenditure and net lending, excluding interest expenditure.

4/ Defined as total revenue minus total expenditure and net lending, excluding externally financed capital expenditure, on-lending, and HIPC/MDRI expenditure.

5/ Defined as expenditures on health, education, environment, the judiciary, social development, sewage and rural irrigation. 
Table 5. Senegal: Monetary Survey, 2004-08

\begin{tabular}{|c|c|c|c|c|c|}
\hline & 2004 & 2005 & 2006 & $\begin{array}{r}2007 \\
\text { Est. }\end{array}$ & $\frac{2008}{\text { Proj. }}$ \\
\hline & \multicolumn{5}{|c|}{ (Billions of CFA francs) } \\
\hline Net foreign assets & 676 & 660 & 780 & 851 & 863 \\
\hline Central Bank of West African States (BCEAO) & 477 & 487 & 569 & 644 & 646 \\
\hline Commercial banks & 199 & 173 & 210 & 207 & 217 \\
\hline Net domestic assets & 770 & 894 & 972 & 1,122 & 1,234 \\
\hline Net domestic credit & 881 & 1,032 & 1,122 & 1,324 & 1,442 \\
\hline Net credit to the government & 24 & -35 & 11 & 96 & 75 \\
\hline Central bank & 108 & 84 & 45 & 55 & 48 \\
\hline Commercial banks & -94 & -123 & -46 & 21 & 8 \\
\hline Other institutions & 10 & 4 & 12 & 20 & 20 \\
\hline Credit to the economy & 857 & 1,067 & 1,111 & 1,228 & 1,367 \\
\hline Of which: crop credit & 14 & 10 & 9 & 10 & 12 \\
\hline Other items (net) & -111 & -138 & -151 & -202 & -208 \\
\hline Broad money (M2) & 1,446 & 1,553 & 1,751 & 1,973 & 2,097 \\
\hline Currency outside banks & 344 & 378 & 453 & 485 & 538 \\
\hline Total deposits & 1,102 & 1,176 & 1,298 & 1,488 & 1,559 \\
\hline Demand deposits & 563 & 593 & 652 & 784 & 821 \\
\hline \multirow[t]{2}{*}{ Time deposits } & 538 & 582 & 646 & 705 & 738 \\
\hline & \multicolumn{5}{|c|}{ (Change in percentage of beginning-of-period broad money stock) } \\
\hline Net foreign assets & 9.8 & -1.2 & 7.7 & 4.1 & 0.6 \\
\hline BCEAO & 9.8 & 0.7 & 5.3 & 4.3 & 0.1 \\
\hline Commercial banks & 0.0 & -1.8 & 2.4 & -0.2 & 0.5 \\
\hline Net domestic assets & 3.1 & 8.6 & 5.0 & 8.6 & 5.7 \\
\hline Net credit to the government & -3.1 & -4.1 & 3.0 & 4.9 & -1.1 \\
\hline Credit to the economy & 5.6 & 14.5 & 2.9 & 6.7 & 7.1 \\
\hline Other items (net) & 0.6 & -1.8 & -0.8 & -2.9 & -0.3 \\
\hline Broad money (M2) & 12.9 & 7.4 & 12.7 & 12.7 & 6.3 \\
\hline Memorandum items: & \multicolumn{5}{|c|}{ (Units indicated) } \\
\hline Velocity (GDP/M2; end of period) & 2.9 & 3.0 & 2.8 & 2.7 & 2.9 \\
\hline Nominal GDP growth (percentage growth) & 6.4 & 8.0 & 5.8 & 10.4 & 12.0 \\
\hline Credit to the economy (percentage growth) & 9.2 & 24.5 & 4.2 & 10.5 & 11.3 \\
\hline Credit to the economy/GDP (percent) & 20.2 & 23.3 & 22.9 & 22.9 & 22.8 \\
\hline Variation of net credit to the government (from & & & & & \\
\hline previous year; billions of CFA francs) & -40.2 & -59.2 & 46.3 & 85.1 & -21.2 \\
\hline Central bank discount rate (end of period; percent) & 4.50 & 4.50 & 4.75 & 4.75 & $\ldots$ \\
\hline
\end{tabular}

Sources: Senegalese authorities; and Fund staff estimates and projections. 
Table 6. Senegal: Millennium Development Goals 1/

\begin{tabular}{|c|c|c|c|c|c|c|c|}
\hline & 1990 & 1995 & 2000 & 2005 & 2006 & 2007 & 2015 \\
\hline \multicolumn{8}{|l|}{ Goal 1. Eradicate extreme poverty and hunger } \\
\hline \multicolumn{8}{|l|}{$\begin{array}{l}\text { Target 1: Halve, between } 1990 \text { and } 2015 \text {, the proportion of people whose } \\
\text { income is less than one dollar a day }\end{array}$} \\
\hline Poverty gap at US\$1 a day (percent) & $\ldots$ & 6 & $\ldots$ & $\ldots$ & $\ldots$ & $\ldots$ & ... \\
\hline National household survey poverty incidence $2 /$ & $\ldots$ & 67.9 & 57.1 & 50.6 & 46 & $\ldots$ & ... \\
\hline Share of income or consumption by poorest 20 percent & 3.5 & 7 & 6.6 & $\ldots$ & $\ldots$ & $\ldots$ & ... \\
\hline \multicolumn{8}{|l|}{$\begin{array}{l}\text { Target 2: Halve between } 1990 \text { and 2015, the proportion of people suffering } \\
\text { hunger }\end{array}$} \\
\hline Prevalence of child malnutrition (percent of children under 5 ) & $\ldots$ & 21.9 & 20.3 & $\ldots$ & $\ldots$ & 15 & {$[10.8$} \\
\hline \multicolumn{8}{|l|}{ Goal 2. Achieve universal primary education } \\
\hline \multicolumn{8}{|l|}{$\begin{array}{l}\text { Target 3: Ensure that, by } 2015 \text {, children will be able to complete a full } \\
\text { course of primary schooling }\end{array}$} \\
\hline Net primary enrollment ratio (percent of relevant age group) & 45.3 & $\ldots$ & 56.5 & 69.6 & 70.7 & $\ldots$ & {$[100.0]$} \\
\hline Youth literacy rate (percent ages 15-24) & 40.1 & $\ldots$ & 49.1 & $\ldots$ & $\ldots$ & $\ldots$ & ... \\
\hline \multicolumn{8}{|l|}{ Goal 3. Promote gender equality and empower women } \\
\hline \multicolumn{8}{|l|}{$\begin{array}{l}\text { Target 4: Eliminate gender disparity in primary and secondary education } \\
\text { preferably by } 2005 \text { and to all levels of education by } 2015\end{array}$} \\
\hline Ratio of girls to boys in primary and secondary education (percent) & 68.5 & $\ldots$ & 83.9 & 90.8 & $\ldots$ & 92 & {$[100.0]$} \\
\hline Ratio of young literate females to males (percent ages 15-24) & 60.4 & $\ldots$ & $\ldots$ & $\ldots$ & $\ldots$ & $\ldots$ & ... \\
\hline Share of women employed in the nonagricultural sector (percent) & 26 & $\ldots$ & $\ldots$ & $\ldots$ & $\ldots$ & $\ldots$ & ... \\
\hline Proportion of seats held by women in national parliament (percent) & 13 & $\ldots$ & 12 & 19 & 19 & 22 & ... \\
\hline \multicolumn{8}{|l|}{ Goal 4. Reduce child mortality } \\
\hline \multicolumn{8}{|l|}{$\begin{array}{l}\text { Target 5: Reduce by two-thirds, between } 1990 \text { and 2015, the under-five } \\
\text { mortality rate }\end{array}$} \\
\hline Under-5 mortality rate (per 1,000 ) & 149 & 148 & 133 & 119 & 116 & 116 & {$[49.3$} \\
\hline Infant mortality rate (per 1,000 live births) & 72 & 72 & 66 & 60 & 60 & 60 & ... \\
\hline Immunization, measles (percent of children under 12 months) & 51 & 80 & 48 & 75 & 80 & 80 & ... \\
\hline \multicolumn{8}{|l|}{ Goal 5. Improve maternal health } \\
\hline \multicolumn{8}{|l|}{$\begin{array}{l}\text { Target 6: Reduce by three-quarters, between } 1990 \text { and 2015, the maternal } \\
\text { mortality rate }\end{array}$} \\
\hline Maternal mortality ratio (modeled estimate, per 100,000 live births) & $\ldots$ & $\ldots$ & $\ldots$ & $\ldots$ & $\ldots$ & 980 & {$[127.5$} \\
\hline Births attended by skilled health staff (percent) & $\ldots$ & 47 & 60 & 60 & 52 & 52 & {$[75]$} \\
\hline \multicolumn{8}{|l|}{ Goal 6. Combat HIVIAIDS, malaria and other diseases } \\
\hline \multicolumn{8}{|l|}{ Target 7: Halt by 2015 , and begin to reverse, the spread of HIVIAIDS } \\
\hline Prevalence of HIV (percent of population aged 15-24) & $\ldots$ & $\ldots$ & 1.0 & 1.5 & 0.4 & 1.0 & ... \\
\hline Number of children orphaned by HIV/AIDS & $\ldots$ & $\ldots$ & $\ldots$ & 25,000 & $\ldots$ & $\ldots$ & ... \\
\hline \multicolumn{8}{|l|}{$\begin{array}{l}\text { Target 8: Halt by } 2015 \text {, and begin to reverse, the incidence of malaria and } \\
\text { other major diseases }\end{array}$} \\
\hline Tuberculosis incidence (per 100,000 people) & 203.3 & $\ldots$ & $\ldots$ & $\ldots$ & 270 & $\ldots$ & ... \\
\hline Tuberculosis cases detected under DOTS (percent) & $\ldots$ & 61 & 54 & $\ldots$ & 48 & 48 & ... \\
\hline \multicolumn{8}{|l|}{ Goal 7. Ensure environmental sustainability } \\
\hline \multicolumn{8}{|l|}{$\begin{array}{l}\text { Target 9: Integrate the principles of sustainable development into policies } \\
\text { and programs. Reverse the loss of environmental resources }\end{array}$} \\
\hline Forest area (percent of total land area) & 49.0 & $\ldots$ & 46.2 & 45 & 45 & $\ldots$ & .. \\
\hline GDP per unit of energy use (2005 PPP U.S. dollar per kilogram oil equivalı & 5 & 6 & 5 & 6.0 & $\ldots$ & $\ldots$ & ... \\
\hline $\mathrm{CO}_{2}$ emissions (metric tons per capita) & 0.4 & 0.4 & 0.4 & $\ldots$ & 0.4 & $\ldots$ & ... \\
\hline \multicolumn{8}{|l|}{$\begin{array}{l}\text { Target 10: Halve by } 2015 \text { proportion of people without access to safe } \\
\text { drinking water }\end{array}$} \\
\hline Access to an improved water source (percent of population) & 67 & 69 & 72 & 77 & $\ldots$ & $\ldots$ & ... \\
\hline \multicolumn{8}{|l|}{$\begin{array}{l}\text { Target 11: Achieve significant improvement in life of at least } 100 \text { million } \\
\text { slum dwellers by } 2020\end{array}$} \\
\hline Access to improved sanitation (percent of urban population) & 26 & 27 & 27 & $\ldots$ & $\ldots$ & 77 & .. \\
\hline \multicolumn{8}{|l|}{ Goal 8. Develop a Global Partnership for Development } \\
\hline \multicolumn{8}{|l|}{ Target 12: Develop and implement strategies for productive work for youth } \\
\hline Fixed line and mobile telephones (per 1,000 people) & 6 & 9 & 44 & 68.5 & 77.2 & 87.8 & ... \\
\hline Personal computers (per 1,000 people) & 2.5 & 7.0 & 15.0 & $\ldots$ & $\ldots$ & $\ldots$ & .. \\
\hline
\end{tabular}

Sources: World Bank staff and World Development Indicators.

$1 /$ The data in italics refer to periods earlier than shown.

2/ Data listed under 2000 are for 2001-02. 
Table 7. Financial Soundness Indicators for the Banking Sector, 2003-08

(Percent, unless otherwise indicated)

\begin{tabular}{|c|c|c|c|c|c|c|}
\hline & $\begin{array}{l}2003 \\
\text { Dec. }\end{array}$ & $\begin{array}{l}2004 \\
\text { Dec. }\end{array}$ & $\begin{array}{l}2005 \\
\text { Dec. }\end{array}$ & $\begin{array}{l}2006 \\
\text { Dec. }\end{array}$ & $\begin{array}{l}2007 \\
\text { Dec. }\end{array}$ & $\begin{array}{r}2008 \\
\text { Jun. }\end{array}$ \\
\hline \multicolumn{7}{|l|}{ Capital Adequacy } \\
\hline Capital to risk weighted assets & 12.1 & 11.9 & 11.1 & 13.1 & 13.6 & 13.7 \\
\hline Regulatory capital to risk weighted assets & 11.7 & 11.5 & 10.8 & 12.9 & 13.5 & 13.5 \\
\hline Capital to total assets & 7.8 & 7.7 & 7.6 & 8.3 & 8.3 & 8.7 \\
\hline \multicolumn{7}{|l|}{ Asset composition and quality } \\
\hline Total loans to total assets & 59.6 & 57.1 & 64.0 & 63.8 & 58.8 & 60.6 \\
\hline $\begin{array}{l}\text { Concentration: loans to } 5 \text { largest borrowers to capital } \\
\text { Sectoral distribution of loans }\end{array}$ & 141.0 & 131.4 & 179.9 & 103.7 & 88.5 & 96.3 \\
\hline Industrial & 41.1 & 33.6 & 35.5 & 28.9 & 25.1 & 22.1 \\
\hline Retail and wholesale trade & 19.9 & 19.3 & 17.0 & 18.9 & 14.4 & 16.4 \\
\hline Services, transportation and communication & 17.2 & 27.4 & 28.0 & 30.0 & 29.6 & 30.2 \\
\hline Gross NPLs to total loans $1 /$ & 13.3 & 12.6 & 11.9 & 16.8 & 18.6 & 19.0 \\
\hline Of which: without ICS & $\ldots$ & .. & $\cdots$ & $\cdot$ & 12.7 & 13.7 \\
\hline Provisions to NPLs 1/ & 75.3 & 75.7 & 75.4 & 52.0 & 53.8 & 50.3 \\
\hline Of which: without ICS & $\ldots$ & $\ldots$ & ... & $\ldots$ & 74.6 & 65.9 \\
\hline NPLs net of provisions to total loans $1 /$ & 3.3 & 3.4 & 3.2 & 8.8 & 8.6 & 9.5 \\
\hline Of which: without ICS & 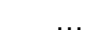 & & & 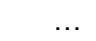 & 3.6 & 5.2 \\
\hline NPLs net of provisions to capital $1 /$ & 27.8 & 25.1 & 27.2 & 67.9 & 60.7 & 65.7 \\
\hline Of which: without ICS & $\ldots$ & $\ldots$ & $\ldots$ & $\ldots$ & 23.8 & 34.0 \\
\hline \multicolumn{7}{|l|}{ Earnings and Profitability } \\
\hline Average cost of borrowed funds & 1.8 & 2.0 & 2.0 & 2.2 & 2.2 & $\ldots$ \\
\hline Average interest rate on loans & 8.7 & 11.7 & 11.8 & 11.3 & 11.6 & $\ldots$ \\
\hline Average interest margin $2 /$ & 6.7 & 9.7 & 9.8 & 9.2 & 9.4 & $\ldots$ \\
\hline After-tax return on average assets & 1.8 & 1.8 & 1.6 & 1.6 & 1.6 & $\cdots$ \\
\hline After-tax return on average equity & 22.1 & 17.6 & 15.8 & 14.6 & 15.3 & $\cdots$ \\
\hline Noninterest expenses/net banking income & 48.9 & 48.7 & 47.9 & 49.4 & 50.7 & $\ldots$ \\
\hline Salaries and wages/net banking income & 21.8 & 21.5 & 21.2 & 21.7 & 22.2 & $\cdots$ \\
\hline \multicolumn{7}{|l|}{ Liquidity } \\
\hline Total deposits to total liabilities & 82.0 & 79.6 & 78.3 & 75.8 & 73.6 & 73.8 \\
\hline
\end{tabular}

Source: BCEAO.

1/ NPL changes in 2006 due to ICS. In 2008, ICS was recapitalized and the government guarantee for its bank loans was lifted. However, the loans in question remain classified as non-performing for the time being, although without the need to provision.

2/ Excluding the tax on banking operations. 
Table 8. Quantitative Assessment Criteria and Indicative Targets, 2007-08 1/

(Billions of CFA francs, unless otherwise specified)

\begin{tabular}{|c|c|c|c|c|c|c|c|c|c|c|c|c|}
\hline & \multicolumn{3}{|c|}{ December 31,2007} & \multicolumn{3}{|c|}{ March 31, 2008} & \multicolumn{3}{|c|}{ June 30,2008} & \multicolumn{3}{|c|}{ September 30, 2008} \\
\hline & $\begin{array}{l}\text { Assessment } \\
\text { criteria }\end{array}$ & Actual & Status & $\begin{array}{l}\text { Indicative } \\
\text { target }\end{array}$ & Actual & Status & $\begin{array}{l}\text { Assessment } \\
\text { criteria }\end{array}$ & Actual & Status & $\begin{array}{l}\text { Indicative } \\
\text { target }\end{array}$ & Actual & Status \\
\hline \multicolumn{13}{|l|}{ Assessment criteria } \\
\hline Floor on the basic fiscal balance $2 /$ & -41 & -53 & not met & -13 & 49 & met & -48 & 17 & met & -71 & -23 & met \\
\hline \multicolumn{13}{|l|}{ Ceiling on the contracting or guaranteeing of new } \\
\hline nonconcessional external debt by the government $3 / 4 /$ & 0 & $\begin{array}{c}0 \\
\text { Arrears }\end{array}$ & met & 0 & 0 & met & 0 & 0 & met & 0 & 0 & met \\
\hline Ceiling on government domestic payment arrears (stock) $5 /$ & 0 & $\begin{array}{l}\text { eliminated } \\
\text { on Nov. } 30\end{array}$ & not met & 0 & 0 & met & 0 & 35 & not met & 0 & $576 /$ & not met \\
\hline Ceiling on government external payment arrears (stock) $5 /$ & 0 & 0 & met & 0 & 0 & met & 0 & 0 & met & 0 & 0 & met \\
\hline \multicolumn{13}{|l|}{ Ceiling on the amount of the float (depenses liquidees } \\
\hline non payees ) $7 /$ & 34 & 55 & not met & 30 & $398 /$ & not met & 50 & $1598 /$ & not met & 40 & $1938 /$ & not met \\
\hline \multicolumn{13}{|l|}{ Indicative target } \\
\hline $\begin{array}{l}\text { Quarterly ceiling on the share of the value of public sector } \\
\text { contracts signed by single tender (in percent) }\end{array}$ & 20 & 29 & not met & 20 & 54 & not met & 20 & 17 & met & 20 & 19 & met \\
\hline
\end{tabular}

1/ Data for March are indicative targets, with the exception of the assessment criteria monitored on a continuous basis.

2/ Defined as total revenue (excluding privatization receipts and sales of mobile telephone licenses) minus total expenditure and net lending, excluding externally financed capital expenditure,

on-lending, and HIPC and MDRI spending. Cumulative since the beginning of the year. The 2007 floor has been adjusted upwards because the recapitalization of La Poste

(for CFAF15.5 bn) did not take place in 2007; floor will be adjusted downwards by this amount in 2008 from the time it is implemented.

$3 /$ Cumulative since approval of first program review in June 2008; continuous before that time.

4/ Excludes government or government-guaranteed CFAF borrowing from financial institutions within WAEMU and

external loans contracted by the airport project company (AIDB) to finance the construction of the new Dakar Airport.

$5 /$ Monitored on a continuous basis.

6/ September 15, 2008

7/ Defined as expenditure for which a bill has been received and recognized (depense liquidee) and which has not been paid; before the approval of

the first program review in June 2008 , defined as the expenditure for which a payment order has been issued and not yet paid.

8/ For budget year 2008 
Table 9. Senegal: Structural Conditionality, May 2008-March 2009

\begin{tabular}{|c|}
\hline Policy Measures \\
\hline Prior Action \\
\hline $\begin{array}{l}\text { 1. Publish administrative orders from the prime } \\
\text { minister and finance minister which will limit the } \\
\text { authorizations for commitments on current } \\
\text { expenditure and domestically-financed capital } \\
\text { expenditure, as specified in paragraph } 10 \text {. }\end{array}$ \\
\hline
\end{tabular}

\section{Structural Assessment Criteria}

2. Adopt and make effective the new tariff structure for electricity, which provides progressive tariff rates, in order to encourage energy savings, with lower tariff adjustments for low-income consumers.

3. Issue the implementation decree for the application of Law 2007-16 on DISEZ, as specified in paragraph 17 of the MEFP.

4. Publish an administrative order from the prime minister with guidelines to strengthen public investment planning and evaluation, as specified in paragraph 16 of the MEFP.

\section{Structural Benchmarks}

5. Develop a rolling two-year program of issuance of government securities, in collaboration with the BCEAO, as specified in paragraph 13 of the MEFP.

6. Complete a comprehensive audit of payment delays by the financial audit inspectorate of the MEF (Inspection Générale des Finances), as specified in paragraph 14 of the MEFP.
Target Date of Implementation

Implementation Status
Implemented on

May 20, 2008
Completed

August 1, $2008 \quad$ Completed

November 15, 2008

Completed

November 15, 2008

Completed
June 30, 2008

Completed on

August 14, 2008 


\begin{tabular}{llc}
\hline \multicolumn{1}{c}{ Policy Measures } & $\begin{array}{l}\text { Target Date of } \\
\text { Implementation }\end{array}$ & Implementation Status \\
\hline $\begin{array}{l}\text { 7. Adopt all the legal texts and regulations allowing } \\
\text { the effective transfer of direct State tax collection } \\
\text { responsibilities from the Treasury to the DGID } \\
\text { effective January 1, 2009, as specified in paragraph }\end{array}$ & October 31, 2008 & Completed \\
23 of the MEFP. & \\
$\begin{array}{l}\text { 8. Prepare memoranda of understanding which } \\
\text { stipulate the respective rights and responsibilities of } \\
\text { APIX, DGID, and DDI regarding the management of } \\
\text { DISEZ, as specified in paragraph 17 of the MEFP. }\end{array}$ & March 31, 2009 \\
\end{tabular}


Table 10. Senegal: Indicators of Capacity to Repay the Fund, 2007-19

\begin{tabular}{|c|c|c|c|c|c|c|c|c|c|c|c|c|c|}
\hline & 2007 & 2008 & 2009 & 2010 & 2011 & 2012 & 2013 & 2014 & 2015 & 2016 & 2017 & 2018 & 2019 \\
\hline & & \multicolumn{12}{|c|}{ Projections } \\
\hline \multicolumn{14}{|l|}{ Obligations to the Fund from existing drawings } \\
\hline Principal (SDR millions) & 0.0 & 0.0 & 0.0 & 0.4 & 2.1 & 3.5 & 3.5 & 3.5 & 3.1 & 1.4 & 0.0 & 0.0 & 0.0 \\
\hline Charges/Interest (SDR millions) & 0.9 & 0.7 & 0.6 & 0.6 & 0.5 & 0.5 & 0.5 & 0.5 & 0.5 & 0.5 & 0.5 & 0.5 & 0.5 \\
\hline \multicolumn{14}{|l|}{ Obligations to the Fund from prospective drawings $1 /$} \\
\hline Principal (SDR millions) & ... & 0.0 & 0.0 & 0.0 & 0.0 & 0.0 & 0.0 & 7.3 & 9.7 & 9.7 & 9.7 & 9.7 & 2.4 \\
\hline Charges/Interest (SDR millions) & ... & 0.0 & 0.2 & 0.2 & 0.2 & 0.2 & 0.2 & 0.2 & 0.2 & 0.1 & 0.1 & 0.1 & 0.0 \\
\hline \multicolumn{14}{|c|}{ Total outstanding and prospective obligations to the Fund 1 / } \\
\hline In millions of SDRs & 0.9 & 0.7 & 0.7 & 1.1 & 2.9 & 4.2 & 4.2 & 11.5 & 13.5 & 11.7 & 10.3 & 10.2 & 2.9 \\
\hline In millions of U.S. dollars & 1.3 & 1.1 & 1.1 & 1.8 & 4.4 & 6.5 & 6.5 & 17.8 & 20.9 & 18.1 & 15.9 & 15.8 & 4.5 \\
\hline In percent of exports of goods and services & 0.1 & 0.0 & 0.0 & 0.0 & 0.1 & 0.2 & 0.1 & 0.4 & 0.4 & 0.3 & 0.3 & 0.3 & 0.1 \\
\hline In percent of debt service & 0.8 & 0.6 & 0.4 & 0.6 & 1.5 & 2.0 & 1.7 & 4.1 & 4.2 & 3.4 & 2.8 & 2.6 & 118.9 \\
\hline In percent of quota & 0.5 & 0.4 & 0.5 & 0.7 & 1.8 & 2.6 & 2.6 & 7.1 & 8.3 & 7.2 & 6.3 & 6.3 & 1.8 \\
\hline In percent of gross official reserves & 0.1 & 0.1 & 0.1 & 0.1 & 0.2 & 0.3 & 0.3 & 0.7 & 0.7 & 0.6 & 0.5 & 0.4 & 0.1 \\
\hline \multicolumn{14}{|l|}{ Total Fund credit outstanding 1/ } \\
\hline In millions of SDRs & 17.3 & 41.6 & 65.9 & 65.5 & 63.4 & 60.0 & 56.5 & 45.8 & 32.9 & 21.8 & 12.1 & 2.4 & 0.0 \\
\hline In millions of U.S. dollars & 27.3 & 63.6 & 101.0 & 100.7 & 97.7 & 92.6 & 87.5 & 70.8 & 50.9 & 33.8 & 18.8 & 3.8 & 0.0 \\
\hline In percent of exports of goods and services & 1.0 & 1.8 & 3.0 & 2.8 & 2.5 & 2.2 & 2.0 & 1.4 & 0.9 & 0.5 & 0.4 & 0.1 & 0.0 \\
\hline In percent of debt service & 15.6 & 34.0 & 39.4 & 35.6 & 32.3 & 28.3 & 22.9 & 16.1 & 10.3 & 6.4 & 3.3 & 0.6 & 0.0 \\
\hline In percent of quota & 10.7 & 25.7 & 40.7 & 40.5 & 39.2 & 37.1 & 34.9 & 28.3 & 20.4 & 13.5 & 7.5 & 1.5 & 0.0 \\
\hline In percent of gross official reserves & 1.8 & 3.8 & 5.6 & 5.4 & 4.9 & 4.2 & 3.7 & 2.8 & 1.8 & 1.1 & 0.6 & 0.1 & 0.0 \\
\hline \multicolumn{14}{|l|}{ Memorandum items: } \\
\hline Exports of goods and services (millions of US\$) & 2,605 & 3,446 & 3,378 & 3,624 & 3,873 & 4,141 & 4,420 & 4,951 & 5,569 & 6,238 & 4,592 & 5,081 & 5,544 \\
\hline Debt service (millions of US\$) & 175 & 187 & 256 & 282 & 303 & 327 & 383 & 438 & 495 & 528 & 567 & 616 & 4 \\
\hline Quota (millions of SDRs) & 162 & 162 & 162 & 162 & 162 & 162 & 162 & 162 & 162 & 162 & 162 & 162 & 162 \\
\hline Gross official reserves (millions of US\$) & 1,535 & 1,672 & 1,817 & 1,851 & 1,990 & 2,186 & 2,348 & 2,572 & 2,815 & 3,067 & 3,407 & 3,800 & 4,202 \\
\hline GDP (millions of US\$) & 11,183 & 13,556 & 13,519 & 14,658 & 15,865 & 17,154 & 18,543 & 19,985 & 21,545 & 23,232 & 25,058 & 27,025 & 29,152 \\
\hline
\end{tabular}

Sources: BCEAO; and Fund staff estimates and projections.

1/ Assuming two disbursements of SDR 24.27 million (15 percent of quota) each under the ESF, in December 2008 and after third review in 2009. 


\section{Senegal-Twelve-Month ArRangement Under The Exogenous Shocks Facility}

Attached hereto is a letter dated December 5, 2008 (the "Letter") from the Minister of Budget, with its attached Memorandum of Economic and Financial Program (the "MEFP") and the Technical Memorandum of Understanding (the "TMU"), requesting from the International Monetary Fund, as Trustee of the Poverty Reduction and Growth Facility and Exogenous Shocks Facility Trust (the "Trustee"), a twelve-month arrangement under the Exogenous Shocks Facility ("ESF") (the "Arrangement"), and setting forth:

(a) the objectives and policies of the program that the authorities of Senegal intend to pursue during the twelve-month period of this Arrangement;

(b) understandings of Senegal with the Trustee regarding reviews that will be made of progress in realizing the objectives of the program.

To support these objectives and policies, the Trustee grants this requested twelve-month Arrangement in accordance with the following provisions, and subject to the provisions applying to assistance under the PRGF-ESF Trust.

1. For a period of twelve months from December 19, 2008, Senegal will have the right to obtain disbursements from the Trustee in a total amount equivalent to SDR 48.54 million, subject to the availability of resources in the PRGF-ESF Trust.

2. During the period of this Arrangement:

(a) the first disbursement, in an amount equivalent to SDR 24.27 million, will be available upon approval of this Arrangement, at the request of Senegal;

(b) the second disbursement, in an amount equivalent to SDR 24.27 million, will be available on or after May 10, 2009, at the request of Senegal and subject to paragraphs 3 and 4 of the Arrangement below.

3. Senegal will not request:

A. the second disbursement under this Arrangement specified in paragraph 2(b) above:

(a) if the Managing Director of the Trustee finds that, with respect to the second disbursement, the data as of December 31, 2008, indicate that:

(i) the floor on the basic fiscal balance, as set out in Table 1 of the MEFP and further specified in the TMU, or

(ii) the ceiling on the amount of the float at the Treasury, as set out in Table 1 of the MEFP and further specified in the TMU, was not observed; 
(b) until the Trustee has determined that, with respect to the second disbursement, the first program review, referred to in paragraph 38 of the MEFP, has been completed.

4. Senegal will not request any disbursement under this Arrangement, if at any time during this Arrangement:

(a) (i) the ceiling on the contracting or guaranteeing of new nonconcessional external debt by the government, or

(ii) the ceiling on the stock of external payment arrears by the government, or

(iii) the ceiling on spending undertaken outside normal and simplified procedures, as specified in Table 1 of the MEFP and further set out in the TMU, is not observed; or

(b) if Senegal

(i) imposes or intensifies restrictions on payments and transfers for current international transactions, or

(ii) introduces or modifies multiple currency practices, or

(iii) concludes bilateral payments agreements that are inconsistent with Article VIII, or

(iv) imposes or intensifies import restrictions for balance of payments reasons.

5. When Senegal is prevented from requesting disbursements under this Arrangement because of paragraphs 3 and 4 of the Arrangement above, such disbursements may be made available only after consultation has taken place between the Trustee and Senegal and understandings have been reached regarding the circumstances in which Senegal may request the disbursements.

6. In accordance with paragraph 4 of the Letter, Senegal will provide the Trustee with such information as the Trustee requests in connection with the progress of Senegal in implementing the policies and reaching the objectives of the program supported by this Arrangement.

7. During the period of this Arrangement, Senegal shall remain in close consultation with the Trustee. In accordance with paragraph 4 of the Letter, Senegal shall consult with the Trustee on the adoption of any measures that may be appropriate at the initiative of the government or whenever the Managing Director of the Trustee requests such a consultation. Moreover, after the period of this Arrangement and while Senegal has outstanding financial obligations to the Trustee arising from loan disbursements under this Arrangement, Senegal will consult with the Trustee from time to time, at the initiative of the government or 
whenever the Managing Director of the Trustee requests consultation on Senegal's economic and financial policies. These consultations may include correspondence and visits of officials of the Trustee to Senegal or of representatives of Senegal to the Trustee. 


\title{
APPENDIX I
}

\section{LETTER OF INTENT}

Dakar, Senegal

December 5, 2008

\begin{abstract}
Mr. Dominique Strauss-Kahn
Managing Director

International Monetary Fund

$70019^{\text {th }}$ Street, N.W.

Washington, D.C., 20431
\end{abstract}

\section{Dear Mr. Strauss-Kahn,}

1. The attached Memorandum of Economic and Financial Policies (MEFP) reviews implementation to date of the government of Senegal's macroeconomic and structural program under the country's three-year Policy Support Instrument (PSI), approved by the IMF Executive Board on November 2, 2007. Details of this program were set out in the initial MEFP of October 3, 2007 and in the MEFP of May 30, 2008. The attached MEFP builds on the two previous Memoranda, with particular emphasis on measures and objectives for the remainder of 2008 and the first half of 2009.

2. The government requests access to the Exogenous Shock Facility (ESF) for 30 percent of quota, the equivalent of SDR 48.54 million, in two tranches. The MEFP outlines the government's policies to respond, in a sustainable manner, to the oil and food price shock that has severely affected Senegal. In particular, the government aims to strengthen agricultural production and implement energy sector reform. It has also eliminated virtually all subsidies and tax suspensions that had been introduced on a temporary basis in the immediate wake of the price shock, and will remove the remaining subsidy on butane gas by mid-2009.

3. Three quantitative assessment criteria for the second review under the PSI were not met, namely those on domestic arrears, budgetary float, and nonconcessional external borrowing. As described in the attached MEFP, the government is taking strong corrective measures to address the underlying weaknesses. In light of these actions and the observance of the other quantitative criteria and structural conditionality under the program, the government requests waivers of the missed criteria. 
4. The government believes that the policies and measures set forth in the attached MEFP are sufficiently strong to achieve the objectives of the PSI program and ESF arrangement. In the government's view, the PSI remains the appropriate vehicle to maintain a close policy dialogue with the IMF, signal commitment to sound policies to the international community, and move the structural reform agenda forward. It will promptly take any additional measures necessary for the achievement of the objectives of the program. The government will consult with the IMF - at its own initiative or whenever the Managing Director of the IMF requests such a consultation-before the adoption of any such measures or changes to the policies described in the attached Memorandum.

5. The government will provide the Fund with such information as the Fund may request in connection with the progress made in implementing the economic and financial policies and achieving the objectives of the program.

6. The government authorizes the IMF to publish this letter, the attached Memorandum, and the related Staff Report.

Sincerely yours, /s/

Mamadou Abdoulaye Sow

Minister of Budget

Attachment: Memorandum of Economic and Financial Policies 


\title{
AtTachment I
}

\section{Memorandum of Economic and Financial Policies}

\author{
Dakar, December 5, 2008
}

\section{INTRODUCTION}

1. The government remains determined to preserve macroeconomic stability, strengthen private sector-driven growth in a sustainable way, and make quick progress toward achieving the Millennium Development Goals. To meet these objectives, the government will implement its economic and financial program, which is based on prudent macroeconomic policies and an acceleration of structural reforms. The commitments made by the government in the Memorandum of Economic and Financial Policies (MEFP) of October 3, 2007, and the MEFP of May 30, 2008, continue to anchor the policies and reforms being pursued. However, these commitments are strengthened in this MEFP, given the observed budgetary weaknesses, so as to prevent such weaknesses in the future and normalize the government's financial relations with the private sector. This MEFP describes recent economic developments, program performance, and the specific measures and objectives envisaged for the remainder of 2008 and the first half of 2009.

\section{Recent Economic Developments and Program Performance}

2. Macroeconomic developments in 2008 were less positive than expected. Despite a sound performance of the agricultural sector, the most recent estimates put GDP growth in 2008 at about 3.9 percent as a result of several factors: (i) the impact of the rise in food and energy prices on real domestic consumption; (ii) the delayed resumption of the operations of Industries Chimiques du Sénégal (ICS); and (iii) a slowdown in private sector activity, especially in the construction sector, related to government payment delays. Inflation is expected to be 6 percent and the current account deficit about 12\% percent of GDP in 2008, mainly because of the large increases in international food prices and, to a lesser extent, energy prices. It is also anticipated that foreign direct investment (FDI) and aid will be adequate to finance a large portion of the balance of payments and maintain a level of reserves to cover around $3 \frac{1}{2}$ months of imports.

3. Budget execution has run into major problems. The difficulties became apparent as a result of Policy Support Instrument (PSI) program measures, in particular: (i) the structural assessment criterion on extending the coverage of the integrated expenditure tracking system (SIGFIP) to the payment phase, (ii) the structural benchmark concerning the audit of payment delays by the financial audit inspectorate (IGF) of the Ministry of Finance, and (iii) the broadening of the definition of the quantitative assessment criterion on the budgetary float. Follow-up work by Ministry of Finance staff has allowed the authorities to 
estimate that the stock of payment delays at end-October 2008 amounted to CFAF 174.9 billion ( 3 percent of GDP) for the regular budget, CFAF 50 billion (3/4 percent of GDP) in Treasury corresponding accounts (comptes de correspondants du Trésor), and CFAF 74 billion (11/4 percent of GDP) of extrabudgetary expenditures, of which by the line ministries CFAF 11 billion, agencies CFAF 31.3 billion, public health institutions CFAF 12.1 billion, and universities CFAF 19.1 billion.

4. The government acknowledges that these amounts result from expenditures that were too high considering the availability of financing and the macroeconomic circumstances prevailing in the country during 2006-08. Three factors explain the problems:

- $\quad$ First, after the surge in oil and food prices the government decided to maintain high energy subsidies (for electricity and butane gas) to protect household purchasing power and, in July 2007, to suspend duties and taxes on some basic staples. This sharply reduced the budgetary room for maneuver. In March 2008, direct subsidies were introduced on rice and cooking oil. All in all, the subsidies on oil and food products represented 7 percent of GDP for the years 2006 to 2008 combined.

- $\quad$ Second, despite the crowding-out pressures from the financial support for oil and food products, the government maintained the implementation speed of its wide-ranging public investment program during the period. This program centers on infrastructure development as an essential pillar for the promotion of growth and success of the poverty reduction strategy (PRSP); it helped sustain economic activity and employment.

- $\quad$ Finally, the frequent use of Treasury advances to undertake spending that not been initially budgeted helped exacerbate cash flow pressures during the budget year. All in all, public expenditure representing 51/2 percent of GDP was executed through Treasury advances in 2006-08, thus reducing available resources for other expenditure.

5. Because of these problems, the government was unable to observe three quantitative assessment criteria: on domestic arrears, the budgetary float, and nonconcessional external borrowing. The nonobservance of the first two criteria resulted directly from the budgetary slippages, while the criterion on nonconcessional external borrowing was intentionally breached to implement the action plan (see paragraph 14) and correct the budgetary slippages. Given the low liquidity in the regional financial market, the government had to resort to nonconcessional external financing to repay the private sector quickly and, thereby, boost economic activity. The government had to do this as the alternative of maintaining a large stock of payment delays would have been harmful to the economy in an already difficult period and would, at any rate, have led to the nonobservance 
of another assessment criterion, on the budgetary float at end-December 2008. The government is seeking waivers from the IMF Executive Board for the noncompliance with these three assessment criteria in view of the strong corrective measures proposed below.

\section{The government confirms, however, that it has observed the other quantitative} criteria of the program. It has not accumulated external arrears throughout the program period. The basic fiscal balance was observed at end-September 2008, as was the indicative ceiling on the share of public sector contracts awarded by single tender, which was contained at 17 percent by end-June and 19 percent by end-September.

\section{In 2008, the government continued its efforts to safeguard social sector} spending. ${ }^{1}$ The fiscal adjustment measures that reduced expenditure by CFAF 191 billion relative to the appropriations approved by the National Assembly in the context of the initial 2008 Budget Law did not affect social sectors. The government plans to increase the total social sector share of the national budget from 32.6 percent in 2007 to 35.9 percent in 2008. It also intends to request technical expertise from the World Bank and other development partners with sectoral expertise to help improve its analysis of the efficiency of social sector spending.

\section{The government has made significant progress in implementing its structural} reform program. All structural assessment criteria and benchmarks had been observed by end-October 2008:

- $\quad$ A plan for issuing treasury bills and government securities was finalized in midAugust, six weeks later than scheduled. The plan is expected to help the Ministry of Finance improve its forecasting of cash flow needs and accustom the market to regular issuances;

- A new electricity tariff structure was adopted at the beginning of August. This is expected to help the national electricity company, SENELEC, restore its financial equilibrium and encourage consumers to save electrical power. The rate increases, which averaged 17 percent, were asymmetrical and relatively small for low-income consumers;

- An audit of domestic payment delays was completed by IGF on September 30, as planned;

\footnotetext{
${ }^{1}$ Social sector spending, as defined in the program, is PRSP expenditure. It includes expenditure on health, education, justice, social development, environmental affairs, rural water works, and sanitation.
} 
- The Council of Ministers adopted, by end-October, all laws and regulations to ensure the effective transfer of direct tax collection responsibilities from the Treasury to the Revenue Authority (DGID);

- In addition, a Prime Minister's circular aimed at improving the system for planning, monitoring, and evaluating public investment was sent to all sectoral ministries and independent executing agencies on November 13, 2008. The circular sets out procedural and institutional rules to ensure: (i) consistency among the various investment projects and between them and strategic objectives; (ii) compatibility between the investment programs and the medium-term macroeconomic framework; and (iii) the establishment and observance of project analysis rules that vary depending on the size of the project, with the aim of selecting the best among them.

\section{MACROECONOMIC POLICIES FOR 2009-10}

\section{The 2009-10 macroeconomic outlook is framed by a relatively unfavorable} international environment. This includes a slowdown in global growth, problems on the financial markets, and the continued rise in food prices. Nevertheless, the government anticipates that the specific structure of the Senegalese economy, combined with the economic policy intentions described below, as well as the elimination of payment delays, will offset the effects of the negative international environment.

- $\quad$ Real GDP growth is projected to average about 51/2 percent over the next two years. This projection is based on the gradual resumption of ICS's production, recovery in private sector activity linked to the elimination of payment arrears, and FDI-related economic growth.

- Inflation is expected to return to its historical level of 2 percent over the medium term. Given the pressures, albeit abating, on food prices over the short term, the government forecasts an inflation rate of $3 \frac{1 / 4}{4}$ percent in 2009. The subregional framework governing monetary and foreign exchange policies will continue to help keep inflation low, based on the peg to the euro. Although the sound performance of the agricultural sector is expected to help reduce inflationary pressures, a prudent fiscal policy will remain the key instrument for achieving macroeconomic stability in Senegal and contributing to the WAEMU's external stability.

- $\quad$ The external current account deficit is projected to fluctuate between 11 percent and 12 percent of GDP and be increasingly financed by FDI flows. External and domestic public debt would increase slightly to about 23 percent and 7 percent of GDP, respectively, by 2010 . 


\section{A. Fiscal Policy}

\section{Fiscal stance}

10. The government reiterates its commitment to limiting the overall fiscal deficit to 4 percent of GDP over the medium term. A deficit of this size will help ensure continued debt sustainability. This will be key for supporting investment and growth, as well as for helping to maintain domestic stability while containing demand pressures and preventing crowding out of the private sector.

11. The deficit will be temporarily lowered to 3.3 percent of GDP in 2008 because of the need to settle payment delays related to 2007 expenditure. The deficit target will be consistent with a basic fiscal deficit of CFAF 21 billion in 2008 (revised quantitative assessment criterion).

12. The government will keep the 2009 fiscal deficit to less than $\mathbf{3}$ percent of GDP. Parliament will pass a 2009 Budget Law that is consistent with the budgetary projections of the macroeconomic framework agreed with the IMF under the PSI (prior action). This will help ensure that appropriations for each ministry (or any other spending body) are consistent with available resources and other macroeconomic objectives of the program. In line with the overall deficit target for 2009, the basic fiscal surplus is expected to exceed CFAF 5 billion at end-June 2009 (quantitative assessment criterion). This surplus is necessary to complete the settlement of all past payment delays.

13. Despite the envisaged fiscal adjustment, expenditure in priority sectors will continue to grow. This should enable the government to increase social spending to 39.2 percent of total expenditure, or 10.5 percent of GDP, by 2010. Social sector spending will reach 9.2 percent of GDP in 2008 and 9.9 percent of GDP in 2009. Expenditure for rural parts of the country and on infrastructure will also be increased. Finally, to facilitate the monitoring of HIPC and MDRI expenditure, the government has expanded the mechanism for identifying such expenditure, which should allow better monitoring in 2009.

\section{Elimination of payment delays}

14. Given the negative impact on private sector activity of the payment delays, in August 2008 the government adopted an action plan aimed at rapidly turning the situation around. The action plan has since been strengthened and now covers several key aspects:

a. The 2008 supplementary budget law passed on November 27, 2008, (i) regularized Treasury advances executed during the year and transfers of appropriations that exceeded the 10 percent threshold authorized by the Organic Budget Law; and (ii) reduced by CFAF 191 billion the appropriations initially approved by parliament to take account of 
revenue shortfalls and incorporate the effect of the expenditure reductions announced by the Prime Minister's circular of May 2008 and the additional reductions of CFAF 100 billion described in the action plan.

b. The government is currently formulating financing options to reduce the budgetary float.

c. To create budgetary room, the government has eliminated all budgetary subsidies on food products. This includes elimination of the subsidy on rice as of October 29, 2008; reinstitution of the previously suspended duties and taxes on other food products as of September 15, 2008; and removal of the specific tax on vegetable oils as of September 15, 2008.

d. The government has undertaken to curtail and then completely eliminate the subsidy on butane gas by end-June 2009, as well as to revise the electricity pricing method (see paragraph 33a).

e. The increase in the levy on alcohol and cigarettes is expected to take effect by endDecember 2008.

f. The government will launch tenders for the privatization of Hotel Méridien Président in the first quarter of 2009 and expects to finalize renegotiation of the second mobile telephone license in 2009.

g. The government will launch technical audits of all extrabudgetary expenditure by endDecember 2008, as described in paragraph 26 below.

h. A major exercise of data reconciliation and verification has been completed by the Treasury, as described in paragraph 24. It will enable the Ministry of Finance to reconcile its data with those of the private sector.

i. As stated below, the government has also committed to taking strong corrective measures to restore the quality and transparency of the budgetary system, prevent new budgetary slippages and, more generally, make public financial management systems compliant with best international practices over the medium term.

15. With a view to quickly regularizing its financial relations with the private sector and preventing the recurrence of budgetary slippages, the government undertakes to:

a. Eliminate domestic payment arrears, as defined by the WAEMU, by end-December 2008 and continuously maintain a zero balance thereafter; 
b. Limit the stock of the budgetary float, defined as expenditure for which bills have been received and validated but not yet paid by the Treasury, to a ceiling of CFAF 92 billion at end-2008 (quantitative assessment criterion);

c. Limit the stock of committed expenditure for which bills have not yet been validated, and strengthen the link between monthly commitments at the level of the sectoral ministries and the availability of cash; and

d. Meet the new quantitative assessment criterion under the program envisaging a zero limit on Treasury advances (to be observed continuously). Expenditures that are not subject to regular budget execution procedures, but are authorized by Advance Decree in line with paragraph 11 of the TMU, are not considered Treasury Advances

\section{Debt management}

16. To ensure debt sustainability, the government will continue to adhere to the general principle of not contracting or guaranteeing external loans on nonconcessional

terms. However, as the government has not yet managed to raise funds totaling about CFAF 80 billion for the Dakar-Diamniadio toll highway because of the difficult situation on international markets, this amount will be raised in the first half of 2009 so that donor resources can be quickly supplemented with the government's financial contribution under the public-private partnership (quantitative assessment criterion). Several feasibility studies, in particular by the World Bank and the French development agency, have shown that the yield of this project is higher than the cost of the government's nonconcessional borrowing. The government will consult with IMF staff well in advance for any further exceptions to this quantitative assessment criterion. Any proceeds from asset sales will in the first place be used to accelerate the settlement of payment delays, extrabudgetary spending accepted by the Ministry of Finance on the basis of technical audits, and repayment of nonconcessional external loans contracted in 2008-09.

17. In accordance with earlier commitments, other aspects of debt management will also be strengthened. First, every quarter or after each issuance, the government will, in consultation with the BCEAO, update the rolling two-year government securities issuance program. Based on the macroeconomic framework of the PSI, the government plans to issue CFAF 30 billion in securities in December 2008 and in each quarter of 2009; it will also update its rolling two-year plan after each issuance. Second, as of December 2008, the halfyearly public debt sustainability analysis will include a study of the risks from contingent liabilities - related to guarantees issued by the government, to PPPs, and to the operations of public enterprises. Finally, the government has requested assistance from the U.S. Treasury to strengthen its debt management capacity. It has also asked the major rating agencies (Standard and Poor's, Fitch, and Moody's) to rate Senegal's country risk so that it can identify the principal sources of weakness and take appropriate remedial measures. 


\section{STRUCTURAL REFORMS}

\section{A. Fiscal Reforms}

18. The government recognizes that the shortcomings in budget planning, execution, and monitoring, as revealed by recent budgetary slippages, must be urgently corrected. This is key to preserving macroeconomic stability, enhancing the efficiency of public spending, ensuring the integrity of the budgetary and accounting systems, and increasing fiscal transparency. The measures described below, based on an IMF technical assistance mission, aim at meeting those objectives and are expected to help prevent the reappearance of past problems. The government will make every effort to implement the recommendations of the technical assistance report, which will be integrated in its public finance reform framework.

\section{Budget planning}

19. Before January 31, 2009, the government will establish by decree the timetable and the main methods for budget formulation, which will be used for the first time for the preparation of the 2010 budget (structural assessment criterion). This will involve, in particular: (i) the preparation of an initial macroeconomic framework by end-March; (ii) the formulation of the government's major economic and fiscal priorities (previous year's budget execution figures, growth forecasts, government revenue and expenditure projections, estimated civil service staffing developments, and other relevant budgetary and financial aggregates) for the fiscal policy debate in parliament, which is to take place by June at the latest; (iii) the establishment of medium-term sectoral expenditure frameworks with a topdown constraint (i.e., expenditure ceilings by ministry and any other administrative unit receiving budgetary appropriations); and (iv) the preparation of a second (updated) macroeconomic framework by end-August for the formulation of the draft budget law.

20. In 2009 the government will introduce two supplementary budgets, one in the middle of the year and one towards the end, or at least one supplementary budget at mid-year. This will make it possible to regularize transfers of appropriations (see paragraph 22) and any extrabudgetary expenditures that the government decides to recognize on the basis of technical audits (see paragraph 26). These supplementary budgets will also regularize all decrees issued for implementation of articles 12, 15, and 18 of the 2001 Organic Budget Law, and revise the estimated use of appropriations (crédits évaluatifs) based on the latest available information. The extent of regularization of extrabudgetary spending accepted by the government based on technical audits will also take into account the availability of resources in 2009; in any case, any remaining regularizations will be undertaken in 2010. The government plans to implement the procedure of two supplementary budgets every year. 


\section{Budget execution}

21. As stated earlier, Treasury advances, which have no legal basis and were one of the reasons for the recent budgetary problems, will be eliminated. The government issued a decree to supplement the general regulation on public accounting (RGCP). The decree limits expenditure execution to the normal and simplified procedures only and prohibits the use of any other type, in particular Treasury advances (completed prior action).

\section{The government will simplify the system for reopening and carrying over} budgetary appropriations and commitments by issuing an implementation decree for articles 17 and 18 of the organic budget law before the end of the complementary period on February 28, 2009 (structural assessment criterion). The current system is excessively complex and too widely used, to the detriment of the principle of annual budgeting and the preservation of macroeconomic stability. The decree will abolish the possibility to reopen appropriations and will set out the system for carrying them over as follows: (i) any capital budget appropriations for which a payment order has not been issued at the end of the complementary period may be carried forward to the subsequent budget year, by order issued no later than 30 days after the end of the complementary period, within an overall ceiling of 5 percent of the total capital budget for the current year (leaving open the choice between carrying out investment planned for the previous year and the current year); (ii) appropriations (crédits de paiement) that are carried over are canceled in the initial budget year and added to the appropriations for the subsequent budget year; (iii) appropriations related to expenditure for which bills have already been received and validated (dépenses liquidées) are automatically carried forward, within the above-mentioned ceiling; and (iv) appropriations not carried forward become unusable. The mid-year supplementary budget law will ensure conformity with the macroeconomic framework agreed with the IMF. In addition, within this new framework, the government will issue an administrative order by March 31, 2009, to definitively settle the reopened and carried-over amounts of appropriations from all budget years prior to 2008, to be borne in the 2009 budget year.

\section{To more systematically implement closure provisions for budget execution and} accounting, the government issued a circular recalling the deadlines. A failure to effectively and expeditiously stop budgetary operations for a given year complicates management of the government's financial position. It also delays compilation of account statements, prevents the accurate assessment of amounts carried forward from one year to the next, undermines the budgeting for subsequent years, and complicates the government's cash flow management. To be able to comply with the required deadlines for the production of documents monitoring the implementation of fiscal policy, the government undertakes to reduce the time allowed for commitments (engagements) by setting the cutoff date at November 30. The complementary period stipulated in the laws, which halts the administrative phase of budget execution (issuance of payment orders) on December 31, and the accounting phase at end-February of the subsequent year, will be applied. Beyond 
February 28, any operation from the previous budget year with an impact on government cash flow must be imputed to the accounts for the current year. A reminder of these measures was issued in a Ministry of Finance circular. The SIGFIP data will be frozen and published in detail on the Ministry of Finance website by April 30 (structural assessment criterion). Any accounting adjustments made after April 30 must be recorded in the following budget year.

\section{Accounting system and budgetary monitoring}

24. The government, in consultation with the IMF, established a detailed procedures manual for reconciling data between the fiscal reporting table (TOFE), the Treasury accounts, the net government position (NGP), and SIGFIP, and between the Treasury accounts and SIGFIP, and published the reconciliation tables for December 2007 and March, May, June, July, August, and September 2008 (completed prior action). This reconciliation will facilitate the rapid detection of budget management problems and increase the accuracy and reliability of the entire budget execution monitoring system. The reconciliation tables were shared with IMF staff and published on the Ministry of Finance website as soon as they were completed. This exercise of reconciliation and publication will subsequently be carried out on a quarterly basis.

25. The government will make every effort to improve SIGFIP as a key budget monitoring system. To that end, the government will undertake an audit of SIGFIP during the first half of 2009 to ensure that each type of expenditure is thoroughly tracked in the system and with the aim of establishing genuine administrative accounting (comptabilité administrative), based on SIGFIP. The government may request technical assistance from the IMF for this audit.

\section{Extrabudgetary expenditure}

26. The IGF audit that led to the identification of CFAF 74 billion in extrabudgetary expenditure will be followed up. An independent external audit will analyze the circumstances under which these commitments were generated. The audit will also analyze the possible existence of additional spending without budgetary lines in addition to the amounts identified by the IGF audit. The terms of reference of this audit will be drawn up in consultation with IMF staff. The audit report will be submitted to IMF staff by end-March 2009 (structural benchmark). No payments will be made to the private sector for any extrabudgetary expenditure before the audit has established the nature of claims and specified the goods and services that were provided and their unit cost. To prevent a recurrence of such spending, the government will impose sanctions on employees found to be at fault and apply a discount factor to the claims of private firms that agreed to provide goods or services on unlawful terms. The government will pay claims recognized on the basis of the audit only after authorization by a budget law providing for simultaneous reductions of appropriations for other expenditure items. 
27. The compilation of the government's Treasury accounts and budget review laws will be accelerated. For this purpose:

- $\quad$ The Treasury accounts for FY 2006 will be forwarded to the Audit Court by endJanuary 2009, those for FY 2007 by end-March 2009, and those for FY 2008 by endJuly 2009, as legally stipulated; and

- $\quad$ The draft budget review laws for FY 2004, FY 2005, FY 2006, and FY 2007 will be sent to the Audit Court by, respectively, end-December 2008, end-February 2009, end-May 2009, and end-June 2009.

\section{Government procurement}

28. A new government procurement framework has been in place since January 1, 2008. The government is determined to apply it rigorously. The principles described in the previous Memorandum remain valid. In particular:

a. The government will meet the quarterly indicative target on the share of government contracts awarded by single tender, set at 20 percent of all contracts, including those entered into by agencies and other government bodies;

b. The government will continue to publish the list of contracts awarded each quarter on the website of the central directorate for public procurement (DCMP);

c. No public procurement will be allowed unless it has been included in the procurement plans submitted to the DCMP; and

d. The procurement regulatory agency (ARMP) will conduct audits and surveys of government contracts, as stated in the previous MEFP. The first audit report for 2008 will be published by May 31, 2009 (structural benchmark).

\section{Large investment projects}

29. The government intends to continue to ensure transparency and efficiency in the implementation of large public investment projects. It deems them essential for increasing the growth potential of the Senegalese economy.

30. Earlier commitments regarding the airport project and the Dakar integrated special economic zone (DISEZ) will be met. In particular, the government will prepare memoranda of understanding spelling out the respective rights and duties of APIX, DGID, and DGD with respect to the management of DISEZ, including conflict resolution rules. They will also describe, among other things, specific measures for combating fraud and tax evasion (structural benchmark for end-March 2009). 


\section{Social safety net for the rise in prices}

\section{Helping the population deal with the rise in food and energy prices is a national}

priority. The government is therefore adopting a balanced approach between the preservation of macroeconomic stability and emergency measures for the needy. This follows the government's decision to eliminate the subsidies and reverse the suspension of customs duties and taxes on certain staples, and to remove the special tax on vegetable oils. More specifically, the government will help the most underprivileged segments of the population through the following measures:

- Expansion of the school meals program. This measure will have a positive impact on the purchasing power of households as well as on enrollment rates and the performance of children in school;

- $\quad$ Feasibility study regarding the introduction of a subsidy on public transportation; and

- $\quad$ Request for technical expertise from the World Bank to analyze the operational feasibility of introducing a targeted program of cash transfers to the poorest households, as recommended by the IMF's Poverty and Social Impact Analysis (PSIA) mission.

32. In the long run, the government will focus on improving the supply and reducing the costs of energy and food products. The agricultural sector is also important for poverty reduction. The government deems it important to increase domestic agricultural production, in particular of high-yield products such as rice. It will therefore work with farmers to improve infrastructure, especially irrigation and storage systems; increase competition; and restructure subsidies with a view to enhancing agricultural productivity. Similarly, energy sector reform aims at boosting production and generating cost reductions that the government plans to gradually pass on to consumers.

\section{Energy sector reform}

33. Energy sector reform is crucial for improving the supply of energy to the economy and containing fiscal risks. The government has started implementing its program of reforms, supported by the World Bank and other donors, as stated in the sectoral policy letter. The government will apply the principle of market-based pricing and of implementing energy saving and efficiency measures. In support of this program, the government will:

a. Modify the structure of electricity tariffs every three months and adjust the institutional price setting process;

b. Complete the recapitalization of SENELEC by mid-2009; CFAF 9 billion will be appropriated in 2009 for this purpose; and 
c. Eliminate the subsidy on butane gas by end-June 2009. To this end, butane gas prices will be adjusted, so that the annual subsidy can be limited to CFAF 32 billion in 2008 and CFAF 8 billion in 2009.

\section{B. Accelerated Growth Strategy and Development of the Private Sector}

34. The Accelerated Growth Strategy (AGS) will remain the anchor for the agenda of reforms and priority actions to enhance the growth potential of the Senegalese economy. The government will continue to adopt a participative approach in the implementation of this strategy, especially with respect to the five cluster sectors. To this end, as stated in the implementation decree of the AGS law, the monitoring committee chaired by the Prime Minister will meet every six months, and the technical committee chaired by the Minister of Finance will meet every three months.

\section{Since the seventh meeting of the Presidential Investment Council (CPI) in} November 2007, Senegal has carried out significant reforms to improve the business climate. The most salient of these have been: (i) simplifying import and export procedures, simplifying customs clearance procedures, and reducing the time and costs for formalities; (ii) simplifying property transfer procedures, with wait times reduced from 114 days to 18 days; (iii) simplifying the procedures for granting building permits, certificates of compliance and other requirements, with wait times reduced from 217 days to 78 days; (iv) passing the law on the promotion and development of small and medium-sized enterprises; (v) improving the legal and judicial framework, especially by increasing the number of magistrates and court clerks; (vi) improving the legal framework applicable to PPPs, for the implementation of infrastructure projects, with the amendment of the law on construction contracts, operations, and infrastructure transfers; (vii) adopting the regulations for implementation of the Labor Code; and (viii) creating new technical and vocational education centers.

36. In the context of the November $2008 \mathrm{CPI}$, the government has committed to taking action in several key areas. These include: (i) updating and modernizing labor legislation, (ii) strengthening vocational education, and (iii) taking stock of the tax system and incentives for the emergence of new methods of financing the private sector, such as leasing, factoring, and venture capital.

\section{Financial Sector Reforms}

37. The government is determined to safeguard the soundness of the financial system and enhance its contribution to the economy. In accordance with its previous commitments, the government therefore intends to: 
a. Implement all the decisions and recommendations of the WAEMU Banking Commission;

b. Act in close cooperation with the BCEAO and the WAEMU Banking Commission to limit the impact of the ICS restructuring plan on the banking system and the budget;

c. Carefully monitor all the potential effects of the global financial crisis on the soundness and liquidity of the Senegalese banking system and the regional financial markets, in close cooperation with the BCEAO, the regional Banking Commission, and the banks' home-country supervisors;

d. Adopt, by end-January 2009, the implementation decree for the new law on microfinance institutions that became effective on September 3, 2008 (structural benchmark), and continue to strengthen the supervision unit of the Ministry of Finance;

e. Submit to Parliament the new legislation on combating the financing of terrorism by end-December 2008; and

f. Continue establishing a legal framework to encourage private sector access to a diversified supply of credit.

\section{Program Monitoring}

38. Quantitative assessment criteria for end-December 2008 and end-June 2009 and quantitative indicative targets for end-March and end-September 2009 were set to monitor program implementation in 2008-09 (see Table 1 in the attached technical memorandum of understanding (TMU)). These program targets will also apply to the ESF. The government and IMF staff also agreed on the prior actions, structural assessment criteria, and structural benchmarks listed in Table 2 of the TMU. The third and fourth reviews under the PSI are scheduled to take place, respectively, by end-June 2009 and end-December 2009, and the first review under the ESF by end-June 2009. The government understands that completion of the third review of the program is contingent upon observance of the assessment criteria set for end-December 2008 and the structural assessment criteria for the period through April 30, 2009. 


\section{ATtaChment II \\ TeChNiCAL Memorandum OF Understanding}

Dakar, December 5, 2008

1. This technical memorandum of understanding (TMU) defines the quantitative and structural assessment criteria, indicative targets, and structural benchmarks on the basis of which the implementation of the Fund-supported program under the Policy Support Instrument (PSI) will be monitored in 2008 and 2009. The quantitative program targets will also serve as performance criteria under the ESF. The TMU also establishes the terms and timeframe for transmitting the data that will enable Fund staff to monitor program implementation.

\section{Program Conditionality}

2. The quantitative assessment criteria for December 31, 2008 and June 30, 2009 and the quantitative indicative targets for March 31, 2009, are shown in Table 1. The prior actions, structural assessment criteria, and structural benchmarks established under the program are presented in Table 2.

\section{Definitions, AdJusters, And DAta Reporting}

\section{A. The Government}

3. Unless otherwise specified below, the government is defined as the central administration of the Republic of Senegal and does not include any local administration, the central bank, or any government-owned entity with a separate legal personality (e.g., public universities and hospitals).

\section{B. Basic Fiscal Balance (Program Definition)}

\section{Definition}

4. The basic fiscal balance (program definition) is the difference between the government's budgetary revenue and total expenditure and net lending, excluding externallyfinanced capital expenditure (financed by donors), drawings on on-lent loans (except on-lent loans to the energy sector financed through donor budget support), and expenditure funded with HIPC- and MDRI-related resources. Budgetary revenue excludes privatization receipts and sales of mobile telephone licenses or other government assets. Government expenditure is defined on the basis of payment orders accepted by the Treasury (dépenses prises en charge par le Trésor). The assessment criterion is set as a floor on the cumulative basic fiscal balance since the beginning of the year. 


\section{Example}

5. The floor for the basic balance (program definition) as at December 31, 2008 is minus CFAF 21 billion. It is calculated as the difference between budgetary revenue (CFAF 1209 billion) and total expenditure and net lending (CFAF 1541 billion), excluding externally financed capital expenditure (CFAF 226 billion), drawings on on-lent loans (CFAF 5 billion), and expenditure funded with HIPC- and MDRI-related resources (CFAF 79 billion).

\section{Reporting requirements}

6. During the program period, the authorities will report monthly to Fund staff provisional data on the basic fiscal balance (program definition) and its components with a lag of no more than 30 days. Data on revenues and expenditure that are included in the calculation of the basic fiscal balance, and on expenditure financed with HIPC- and MDRIrelated resources, will be drawn from preliminary treasury account balances. Final data will be provided as soon as the final balances of the treasury accounts are available, but not later than two months after the reporting of the provisional data.

\section{Government Domestic Payment Arrears}

\section{Definition}

7. In line with the WAEMU definition, domestic payment arrears are government expenditures cleared for payment (dépenses ordonnancées) but not paid during a period of 90 days after the date the payment order (ordonnancement) was cleared. The assessment criterion on domestic payment arrears will be monitored until the time of the second PSI review on a continuous basis and discontinued thereafter.

\section{Reporting requirements}

8. The authorities will report to Fund staff any accumulation of domestic payment arrears, as defined above, as soon as incurred.

\section{Budgetary Float}

\section{Definition}

9. The budgetary float (instances de paiement) is defined as the outstanding stock of government expenditure for which bills have been received and validated but not yet paid by the Treasury (the difference between dépenses liquidées non encore ordonnancées and dépenses payées). The assessment criterion is set as a ceiling on the budgetary float, monitored at the end of the quarter. 


\section{Reporting requirements}

10. The authorities will report to Fund staff on a weekly basis (i.e., at the end of each week), and at the end of each month, a table from the expenditure tracking system (SIGFIP) showing all committed expenditures (dépenses engagées), all certified expenditures that have not yet been cleared for payment (dépenses liquidées non encore ordonnancées), all payment orders (dépenses ordonnancées), all payment orders accepted by the Treasury (dépenses prises en charge par le Trésor), and all payments made by the Treasury (dépenses payées). The SIGFIP table will exclude delegations for regions and embassies and treasury advances (separating regularized and nonregularized), which will be provided in a separate table. The SIGFIP table will also list any payments that do not have a cash impact on the Treasury accounts.

\section{E. Spending Undertaken Outside of Simplified and Normal Procedures}

11. This criterion is applied on a continuous basis to any procedure other than simplified and normal procedures to execute spending, including in particular Treasury advances. It only excludes spending undertaken on the basis of an advance decree for absolute urgency and need in the national interest, based on Article 12 of the Organic Budget Law. Such spending requires signatures by the President and Prime Minister. The criterion is monitored effective the time of the second PSI review.

12. The authorities will report to Fund staff on a monthly basis and with a maximum delay of 30 days any such procedure, together with the SIGFIP table defined in paragraph 10.

\section{F. Government External Payment Arrears}

\section{Definition}

13. External payment arrears are defined as the sum of payments owed and not paid on the external debt contracted or guaranteed by the government. The definition of external debt given in paragraph 15 is applicable here. The assessment criterion on external payment arrears will be monitored on a continuous basis.

\section{Reporting requirements}

14. The authorities will report to Fund staff any accumulation in external payment arrears as soon as the due date is passed. 


\section{G. Contracting or Guaranteeing of New Nonconcessional External Debt by the Government}

\section{Definition}

15. This assessment criterion applies not only to debt as defined in Point No. 9 of the Guidelines on Performance Criteria with Respect to Foreign Debt (Executive Board Decision No. 6230-(79/140), last amended by Executive Board Decision No. 12274-(00/85), adopted August 24, 2000, but also to commitments contracted or guaranteed by the government for which funds have not been received. The criterion does not apply to:

(i) CFAF debt contracted or guaranteed by the government with WAEMU residents;

(ii) CFAF debt initially contracted or guaranteed by the government with WAEMU residents subsequently acquired by nonresidents;

(iii) CFAF government or government-guaranteed debt where the agreement is between the government and a resident WAEMU entity and there is no ensuing contractual obligation between the government and a nonresident entity, regardless of whether the resident WAEMU entity resells the debt to a non-resident;

(iv) debt rescheduling transactions of debt existing at the time of the approval of the PSI; and

(v) external debt contracted by the airport project company (AIDB) to finance construction of the new Dakar Airport.

16. This criterion is measured on a cumulative basis since the approval of the second program review and applies continuously. The ceiling is raised to accommodate CFAF 80 billion to finance exclusively the Dakar-Diamniadio toll highway project. No adjuster will apply to this criterion.

17. For purposes of this assessment criterion, government is understood to include the government as defined in paragraph 3 above, as well as public institutions of an industrial and commercial nature (EPIC), public administrative institutions (EPA), public institutions of a scientific and technical nature, public institutions of a professional nature, public health institutions, local administrations, public enterprises, and government-owned or controlled independent companies (sociétés nationales) (i.e., public enterprises with financial autonomy where the government holds at least 50 percent of the capital), and government agencies.

18. Any external debt of which the present value, calculated with the reference interest rates mentioned hereafter, is greater than 65 percent of the nominal value (grant element of less than 35 percent) is considered nonconcessional, with the exception of IMF lending under the Poverty Reduction and Growth Facility, which is considered concessional even if it does not meet the 35 percent grant element threshold. For debt with a maturity of more than 
15 years, the ten-year reference market interest rate, published by the OECD, is used to calculate the grant element. The six-month reference market rate is used for debt with shorter maturities.

\section{Reporting requirements}

19. The government will report any new external borrowing and its terms to Fund staff as soon as external debt is contracted or guaranteed by the government.

\section{H. Public Sector Contracts Signed by Single Tender}

\section{Definition}

20. Public sector contracts are administrative contracts, drawn up and entered into by government entities subject to the procurement code, for the procurement of supplies, delivery of services, or execution of work. Public sector contracts are considered singletender contracts when the contracting agent signs the contract with the chosen contractor without competitive tender or award. The quarterly indicative target will apply to public sector contracts examined by the Commission Nationale des Contrats de l'Administration (CNCA) until December 31, 2007, and to those examined by the Direction Centrale des Marchés (DCM) thereafter.

\section{Reporting requirements}

21. The government will report quarterly to Fund staff, with a lag of no more than one month from the end of the observation period, the total value of contracts signed by all ministries and agencies and the total value of all single-tender contracts signed by these ministries and agencies.

\section{AdDitional Information for Program Monitoring}

22. The authorities will report to Fund staff the following, with the maximum time lags indicated:

(a) Effective immediately: any decision, circular, edict, decree, ordinance, or law having economic or financial implications for the current program;

(b) With a maximum lag of 30 days, preliminary data on:

- Tax receipts and tax and customs assessments by categories, accompanied by the corresponding revenue collected by the Treasury on a monthly basis;

- The monthly amount of expenditures committed, certified, and for which payment orders have been issued; 
- The quarterly report of the Debt and Investment Directorate (DDI) on execution of investment programs;

- The monthly preliminary government financial operations table (TOFE), based on the Treasury accounts (balances de compte);

- The provisional balance of the Treasury accounts; and

- A reconciliation table between the fiscal reporting table (TOFE), the Treasury accounts (identifying the relevant accounts and amounts), the net government position (NGP), and the SIGFIP on a quarterly basis.

(c) Final data will be provided as soon as the final balances of the treasury accounts are available, but not later than one month after the reporting of provisional data.

23. During the program period, the authorities will report to Fund staff provisional data on a monthly basis on current nonwage non-interest expenditures and domestically financed capital expenditures executed through advance payments and treasury advances, with a lag of no more than 30 days. The data will be drawn from preliminary consolidated treasury account balances. Final data will be provided as soon as the final balances of the treasury accounts are available, but no more than one month after the reporting of provisional data.

24. The government will report to Fund staff:

- The monthly balance sheet of the Central Bank, with a maximum lag of one month;

- The consolidated balance sheet of banks with a maximum lag of two months;

- The monetary survey, on a quarterly basis, with a maximum lag of two months;

- The lending and deposit interest rates of commercial banks, on a monthly basis; and

- Prudential supervision and financial soundness indicators for bank and nonbank financial institutions, as reported in the Table entitled Situation des Etablissements de Crédit vis-àvis du Dispositif Prudentiel [Survey of Credit Institutions in Relation to the Prudential Framework], on a quarterly basis.

The government will update monthly on the website used for this purpose the amount of airport tax — redevance de développement des infrastructures aéroportuaires (RDIA) collected, deposited in the escrow account, and used for the repayment of the loan financing the construction of the new airport. 
MEFP Table 1. Senegal: Quantitative Assessment Criteria (Performance Criteria) and Indicative Target for 2008-09 1/

(Billions of CFA francs, unless otherwise specified)

\begin{tabular}{|c|c|c|c|c|c|c|}
\hline & \multicolumn{2}{|c|}{ December 31, 2008} & $\begin{array}{l}\text { March 31, } \\
2009\end{array}$ & $\begin{array}{c}\text { June } 30 \\
2009\end{array}$ & $\begin{array}{l}\text { September } \\
30,2009\end{array}$ & $\begin{array}{c}\text { December } \\
31,2009 \\
\end{array}$ \\
\hline & Existing & \multicolumn{5}{|c|}{ Proposed } \\
\hline \multicolumn{7}{|l|}{ Assessment criteria } \\
\hline Floor on the basic fiscal balance $2 /$ & -95 & -21 & 2 & 5 & 7 & 10 \\
\hline \multicolumn{7}{|l|}{ Ceiling on the contracting or guaranteeing of new } \\
\hline nonconcessional extemal debt by the government $3 / 4$ / & $806 /$ & $807 /$ & 8071 & $807 /$ & $807 /$ & 8071 \\
\hline $\begin{array}{l}\text { Ceiling on government domestic payment arrears (stock) } 4 / 5 / \\
\text { Ceiling on spending undertaken outside nomal and simplified }\end{array}$ & 0 & $\ldots$ & $\ldots$ & $\ldots$ & $\ldots$ & $\ldots$ \\
\hline procedures $4 / 8 /$ & $\cdots$ & 0 & 0 & 0 & 0 & 0 \\
\hline Ceiling on government external payment arrears (stock) 4/ & 0 & 0 & 0 & 0 & 0 & 0 \\
\hline \multicolumn{7}{|l|}{ Ceiling on the amount of the float (depenses liquidees } \\
\hline non payees par le Tresor) 9/ & 30 & 92 & 80 & 30 & 30 & 30 \\
\hline \multicolumn{7}{|l|}{ Indicative target } \\
\hline $\begin{array}{l}\text { Quarterly ceiling on the share of the value of public sector } \\
\text { contracts signed by single tender (in percent) }\end{array}$ & 20 & 20 & 20 & 20 & 20 & 20 \\
\hline
\end{tabular}

1/ Data for March and September 2009 are indicative targets, with the exception of the assessment criteria monitored on a continuous basis.

2/ Defined as total revenue minus total expenditure and net lending, excluding externally financed capital expenditure, on-lending,

and HIPC and MDRI spending. Cumulative since the beginning of the year. Total revenue excludes

privatization receipts and sales of mobile telephone licenses.

$3 /$ This criterion excludes government or government-guaranteed CFAF borrowing from financial institutions within WAEMU. It also excludes external loans contracted by the airport project company (AIDB) to finance the construction of the new Dakar Airport.

4/ Monitored on a continuous basis.

5/ Discontinued as of the time of second review, since the broader AC on the budgetary float applies.

$6 /$ This target is defined on a cumulative basis since the approval of the first program review. The ceiling was raised to CFAF 80 billion in the fourth quarter of 2008 , to finance exclusively the Dakar-Diamniadio toll highway project. The CFAF 80 billion ceiling will be adjusted downward by the amount of any receipt from a privatization, a sale of a telecommunications license, or any other State asset (such as Sonatel shares) during 2008.

7/ This target is defined on a cumulative basis since the approval of the second program review. The amount of up to CFAF 80 billion

is to finance exclusively the Dakar-Diamniadio toll highway project.

8/ Criterion applies from the time of the second PSI review.

9/ The budgetary float is defined as all the expenditure for which a bill has been received and recognized (depense liquidee) but which has not yet been paid by the treasury. 
TABLE 2 OF THE MEFP: STRUCTURAL CONDITIONALITY, DECEMBER 2008-JUNE 2009

Policy Measures
Prior Action
1. Ensure passage by Parliament of the 2009 Budget Law,
as described in paragraph 12 of the MEFP.
as described in paragraph 12 of the MEFP.

2. Adopt a decree amending the general regulations on government accounting (RGCP) to require use of only the normal and simplified procedures, and prohibiting the use of procedures of any other type, in particular Treasury advances, as described in paragraph 21 of the MEFP.

3. Set out in a manual the detailed procedures for reconciling the budget, the TOFE, the Treasury accounts, the NGP, and the SIGFIP; establish this reconciliation for end-2007 and selected months in 2008, and publish the results, as described in paragraph 24 of the MEFP.

\section{Structural Assessment Criteria}

1. Improve the budget procedure by adopting a decree to set the timetable and principal methods for compiling the budget, as described in paragraph 19 of the MEFP.

2. Adopt a decree specifying the system for the reopening and carryover of budgetary appropriations and commitments, as described in paragraph 22 of the MEFP.

3. Close the 2008 budget and accounting year by freezing and publishing the SIGFIP by April 30, 2009, as described in paragraph 23 of the MEFP.
December 15, 2008 Ensure that the budget is consistent with the macroeconomic framework and resource availability.

$\begin{array}{cl}\begin{array}{c}\text { Completed on } \\ \text { November 28, 2008 }\end{array} & \begin{array}{l}\text { Ensure macroeconomic stability } \\ \text { and the transparency of budgetary } \\ \text { procedures, while addressing one } \\ \text { of the main causes of past } \\ \text { budgetary slippages. }\end{array} \\ \begin{array}{c}\text { Completed on } \\ \text { November 30, 2008 }\end{array} & \begin{array}{l}\text { Restore the reliability of the } \\ \text { budget monitoring systems to } \\ \text { facilitate fiscal policy assessment. }\end{array}\end{array}$

January 31, 2009 Ensure that the budget is formulated in a way that is consistent with macroeconomic circumstances and the available resources.

February 28, 2009 Improve the monitoring of budget execution and increase the observance of the one-year budget rule.

April 30, 2009

Ensure that the account statement allows for an accurate assessment of expenditure carryovers from one year to the next and facilitate government cash flow management. 


\begin{tabular}{ccc}
\hline Policy Measures & $\begin{array}{c}\text { Target Date for } \\
\text { Implementation }\end{array}$ & Macroeconomic Rationale \\
\hline
\end{tabular}

\section{Structural Benchmarks}

1. Adopt the decree implementing the new law on microfinance institutions.

2. Conduct an external audit of all extrabudgetary commitments, based on terms of reference drawn up in consultation with IMF staff, as described in paragraph 26 of the MEFP.

3. Draft memoranda of understanding specifying the respective rights and duties of the APIX, DGID, and DDI regarding the management of the DISEZ, as stated in paragraph 30 of the MEFP.

4. Compile and publish the first audit report of the Government Procurement Regulatory Authority (ARMP), as described in paragraph 28 of the MEFP.
January 31, 2009 Enhance financial intermediation and provide better access to credit, putting into effect the new regulatory and prudential framework for this rapidly growing sector which provides one-tenth of all credit to the private sector.

March 31, 2009 Create the basis for rejecting or regularizing extrabudgetary expenditure in a supplementary budget.

March 31, 2009 Safeguard government revenue.

May 31, 2009

Increase fiscal transparency and governance. Assess the functioning of the new government procurement framework. 
INTERNATIONAL MONETARY FUND

\section{SENEGAL}

Second Review Under the Policy Support Instrument, Request for a Twelve-Month Arrangement Under the Exogenous Shocks Facility, and Request for Waivers and Modification of Assessment CriteriaInformational Annex

Prepared by the African Department

(In collaboration with other departments)

Approved by Benedicte Vibe Christensen and Anthony R. Boote

December 8, 2008

- Relations with the Fund. Describes financial and technical assistance by the IMF and provides information on the safeguards assessment and exchange system. Outstanding purchases and loans amounted to SDR 17.33 million (10.7 percent of quota) at end-October 2008.

- Relations with the World Bank. Describes Bank and Fund work program.

- $\quad$ Article VIII Status. Senegal accepted the obligations of Article VIII of the Fund's Articles as of June 1, 1996 and maintains an exchange system free of restrictions on the making of payments and transfers for current international transactions.

- $\quad$ Statistical Issues. Data provision has some shortcomings, but is broadly adequate for surveillance and program monitoring. There are weaknesses in data on national accounts, production, and social indicators. 
Senegal: Relations with the Fund

(As of October 31, 2008)

I. Membership Status: Joined: August 31, 1962; Article VIII as of June 1, 1996.

II. General Resources Account:

Quota

Fund holdings of currency

Reserve Position

III. SDR Department:

Net cumulative allocation

Holdings

IV. Outstanding Purchases and Loans:

PRGF Arrangements
SDR Million

161.80

160.16

1.67

SDR Million

24.46

0.22

SDR Million

17.33
Percent of Quota

100.00

98.98

1.03

Percent of Allocation

100.00

0.91

Percent of Quota

10.71

V. Latest Financial Arrangements:

\begin{tabular}{lcccc} 
Type & $\begin{array}{c}\text { Date of } \\
\text { Arrangement }\end{array}$ & $\begin{array}{c}\text { Expiration } \\
\text { Date }\end{array}$ & $\begin{array}{c}\text { Amount Approved } \\
\text { (SDR Million) }\end{array}$ & $\begin{array}{c}\text { Amount Drawn } \\
\text { (SDR Million) }\end{array}$ \\
\cline { 2 - 3 } & Apr 28, 2003 & Apr 27, 2006 & 24.27 & 24.27 \\
PRGF & Apr 20, 1998 & Apr 19, 2002 & 107.01 & 96.47 \\
PRGF & Aug 29, 1994 & Jan 12,1998 & 130.79 & 130.79
\end{tabular}

\section{Projected Payments to Fund:}

(SDR Million; based on existing use of resources and present holdings of SDRs):

Principal

Charges/Interest

Total
Forthcoming

\begin{tabular}{lllll}
\hline 2008 & $\underline{2009}$ & $\frac{2010}{0.35}$ & $\frac{2011}{2.08}$ & $\frac{2012}{3.47}$ \\
$\frac{0.21}{0.21}$ & $\underline{0.55}$ & $\underline{0.55}$ & $\underline{0.54}$ & $\underline{0.53}$ \\
\hline 0.55 & 0.90 & & 2.62 & 3.99
\end{tabular}




\section{Implementation of HIPC Initiative: ${ }^{1}$}

Enhanced

I. Commitment of HIPC assistance

Framework

Decision point date

June 2000

Assistance committed

by all creditors (US\$ million)

488.30

Of which: IMF assistance (US\$ million)

(SDR equivalent in millions)

Completion point date

April 2004

II. Disbursement of IMF assistance (SDR million)

Assistance disbursed to the member

Interim assistance

Completion point balance

Additional disbursement of interest income ${ }^{2}$

Total disbursements

\section{Implementation of MDRI Assistance:}

I. Total debt relief (SDR million) ${ }^{3}$

100.32

Of which: MDRI

94.76

HIPC

II. Debt relief by facility (SDR million)

\section{Eligible Debt}

$\begin{array}{lrrr}\frac{\text { Delivery }}{\underline{\text { Date }}} & \underline{\text { GRA }} & \underline{\text { PRGF }} & \underline{\text { Total }} \\ \text { January } 2006 & \text { N/A } & 100.32 & 100.32\end{array}$

\footnotetext{
${ }^{1}$ Assistance committed under the original framework is expressed in net present value (NPV) terms at the completion point, and assistance committed under the enhanced framework is expressed in NPV terms at the decision point. Hence, these two amounts cannot be added.

${ }^{2}$ Under the enhanced framework, an additional disbursement is made at the completion point corresponding to interest income earned on the amount committed at the decision point but not disbursed during the interim period.

${ }^{3}$ The Multilateral Debt Relief Initiative (MDRI) provides 100 percent debt relief to eligible member countries that are qualified for the assistance. The debt relief covers the full stock of debt owed to the Fund as of end2004 which remains outstanding at the time the member qualifies for such debt relief. The MDRI is financed by
} 


\section{Safeguards Assessments:}

The Central Bank of West African States (BCEAO) is the common central bank of the countries of the West African Economic and Monetary Union, which includes Senegal. The most recent safeguards assessment of the BCEAO was completed on November 4, 2005. The assessment indicated progress has been made in strengthening the bank's safeguards framework since the 2002 assessment and identified some areas where further steps would help solidify it.

The BCEAO now publishes a full set of audited financial statements and improvements have been made to move financial reporting closer to International Financial Reporting Standards (IFRS). Furthermore, an internal audit charter has been put in place, mechanisms for improving risk management have been established, and follow-up on internal and external audit recommendations has been strengthened.

The results of continuous safeguards monitoring indicate that while certain vulnerabilities remain in internal control systems and legal structure, there has been some progress in other areas, including through: (i) improving the external audit process by adopting a multi-year audit program; (ii) establishing an audit committee; (iii) expanding disclosures on financial positions of WAEMU countries with the Fund in the notes to the annual financial statements; and (iv) further strengthening of the effectiveness of the internal audit function.

\section{Exchange System:}

Senegal is a member of the West African Economic and Monetary Union (WAEMU). The exchange system, common to all members of the union, is free of restrictions on the making of payments and transfers for current international transactions. The union's common currency, the CFA franc, had been pegged to the French franc at the rate of CFAF $1=\mathrm{F} 0.02$. Effective January 12, 1994, the CFA franc was devalued and the new parity set at CFAF $1=$ F 0.01. Effective December 31, 1998, the parity was switched to the euro at a rate of CFAF $655.96=€ 1$.

The authorities confirmed that Senegal had not imposed measures that could give rise to exchange restrictions subject to Fund jurisdiction. They will inform the Fund if any such measure is introduced.

Aspects of the exchange system are also discussed in the recent report on economic developments and regional policy issues of the WAEMU.

\section{Article IV Consultations:}

The 2008 Article IV consultation was completed by the Executive Board on June 18, 2008 (IMF Country Report No. 08/209). The next consultation is expected to be held within 24 months. In concluding the 2008 Article IV consultation, Executive Directors encouraged

bilateral contributions and the Fund's own resources, as well as the resources already disbursed to the member under the HIPC Initiative (see Section VII above). 
the authorities to maintain prudent macroeconomic policies and persevere in implementing their structural reforms to encourage private-sector led growth. They considered that Senegal's sluggish export performance over the last decade was largely related to structural impediments in the economy, and encouraged the authorities to improve the business environment to make it more conducive to private-sector led growth, raise external competitiveness, and strengthen and diversify exports. They underlined that it was critical to contain the fiscal deficit to preserve debt sustainability, respect the limited financing capacity of the regional financial market, and help contribute to domestic stability in the WAEMU. Directors urged the careful review and expeditious settlement of payment delays, with a view to rigorously applying the existing budget framework. They welcomed the authorities' commitment to continue public financial management reform.

\section{Financial Sector Assessment Program (FSAP) and Report on the Observance of Standards and Codes (ROSC) Participation:}

A joint team of the World Bank and the International Monetary Fund conducted a mission under the FSAP program in November 2000 and January 2001. The Financial System Stability Assessment (FSSA) was issued in August 2001 (IMF Country Report No. 01/189). An FSAP update was undertaken in June 2004, focusing on development issues (in particular nationwide supply of basic financial services and access of SMEs to credit), in line with the priorities defined in the PRSP (IMF Country Report No. 05/126). A regional FSAP for the WAEMU was undertaken in the second half of 2007.

A ROSC on the data module, based on a September 2001 mission, was published on December 2, 2002. An FAD mission conducted a ROSC on the fiscal transparency module in January 2005.

\section{Technical Assistance:}

\section{A. AFRITAC West}

Area

Focus

2003 Debt management and financial markets

Microfinance

2004 Public expenditure management Debt management and financial markets

Public expenditure management Debt management and financial markets

2005 Macroeconomic statistics

Microfinance
Upgrading of information systems; techniques of external debt management

Initiate work with BCEAO and donors

Workshop

Evaluation of software for improving debt management; workshop on AFL/CFT

Decentralization; evaluation of TA needs

Assess need for capacity improvement

Make fiscal data conform to WAEMU and other international norms

Inspection and control; workshop on good governance; training of government supervisory personnel 


\begin{tabular}{lll}
\hline \multicolumn{1}{c}{ Area } & \\
\hline \multirow{2}{*}{2006} & Customs administration & Software risks \\
& Fiscal administration & Reforms and TA needs \\
& Macroeconomic statistics & Evaluate implementation of prior TA and future needs \\
& National accounts & Work program for improvement and statistical action plan \\
& Microfinance & Supervision \\
& Customs administration & Risk analysis and control \\
& Fiscal administration & Modernization \\
& Debt management and financial & Assess TA needs; regional workshop on external debt \\
& markets & statistics \\
& Macroeconomic statistics & Public finance statistics \\
& National accounts & Institutional sectors and quarterly national accounts; \\
& Microfinance & regional workshop on government accounts \\
& Debt management and financial & DSA workshop \\
& markets & \\
National accounts & Institutional sector accounts \\
& Microfinance & Supervision and organization \\
\end{tabular}

\section{B. Headquarters}

$\begin{array}{lll}\text { Department } & \text { Date } & \text { Purpose }\end{array}$

$\begin{array}{ll}\text { February 2004 } & \text { Staff } \\ \text { November 2004 } & \text { Staff } \\ \text { January 2005 } & \text { Staff } \\ \text { February 2006 } & \text { Staff } \\ \text { January 2008 } & \text { Staff } \\ \text { February 2008 } & \text { Staff } \\ \text { October 2008 } & \text { Staff/AFRITAC }\end{array}$

Monetary and September 2006 Staff Capital Markets

Statistics

September 2001 Staff

Fiscal Affairs September 2001 Staff/consultant

Assessment of capacity to track povertyreducing expenditures

Fiscal reporting

PSIA - Poverty and social impact analysis

ROSC

Tax administration

Public-Private Partnerships

Poverty and social impact analysis

Public financial management

Bank supervision and regulation

ROSC assessment of data

July 2002

AFRISTAT
Real sector statistics assessment. Mission under GDDS West Africa project 


\begin{tabular}{|c|c|c|c|}
\hline Department & Date & Form & Purpose \\
\hline & August 2002 & AFRISTAT & $\begin{array}{l}\text { National accounts assistance under GDDS } \\
\text { West Africa project. }\end{array}$ \\
\hline & August 2002 & $\begin{array}{l}\text { Regional } \\
\text { advisor }\end{array}$ & $\begin{array}{l}\text { Continued assistance with fiscal sector data } \\
\text { under GDDS West Africa project. }\end{array}$ \\
\hline & December 2002 & AFRISTAT & $\begin{array}{l}\text { Continued assistance with national } \\
\text { accounts and prices statistics under GDDS } \\
\text { West Africa project }\end{array}$ \\
\hline & February 2003 & $\begin{array}{l}\text { Regional } \\
\text { advisor }\end{array}$ & $\begin{array}{l}\text { Continued assistance with fiscal sector data } \\
\text { under GDDS West Africa project. }\end{array}$ \\
\hline & March 2006 & Staff & Real sector statistics \\
\hline & March 2006 & Staff & Government finance statistics \\
\hline & November 2008 & Staff & SDDS assessment \\
\hline
\end{tabular}

\section{Resident Representative}

Stationed in Dakar since July 24, 1984. The position has been held by Mr. Alex SeguraUbiergo since September 22, 2006.

\section{Anti Money Laundering / Combating the Financing of Terrorism}

The onsite visit for Senegal's AML/CFT evaluation took place in July/August 2007 in the context of ECOWAS Inter-Governmental Action Group Against Money Laundering (GIABA). The report was adopted in early May 2008 by the GIABA Plenary held in Accra, Ghana. The report highlights several areas of weaknesses in the AML/CFT system, confirmed by the score of 12 Non-Compliant and 17 Partially Compliant ratings out of the 40+9 FAF AML/CFT recommendations. Table 2 annexed to the Report contains recommendations of the areas which the authorities should address in order to strengthen the AML/CFT system. In order to strengthen Senegal's AML/CFT framework, the authorities should address the shortcomings identified by the GIABA in the May 2008 evaluation report.

\section{Fourth Amendment of the Articles of Agreement and the Eleventh Quota Review}

The authorities have indicated their agreement with the Fourth Amendment of the Articles of Agreement. The increase in Senegal's quota under the Eleventh General Review of Quotas was completed on February 11, 1999. 


\section{Senegal-Work Program of World Bank and International Monetary Fund, March 2008-June 2009}

\begin{tabular}{|c|c|c|c|}
\hline Title & Products & $\begin{array}{c}\text { Provisional timing of } \\
\text { missions }\end{array}$ & Expected delivery date \\
\hline \multicolumn{4}{|c|}{ A. Mutual information on relevant work programs } \\
\hline World Bank & $\begin{array}{l}\text { Energy Development Policy } \\
\text { Lending } \\
\text { Poverty Assessment } \\
\text { Dakar-Diamniadio toll road } \\
\text { Poverty Reduction Support } \\
\text { Credit IV } \\
\text { Country Assistance Strategy } \\
\text { Progress Report } \\
\text { Public Expenditure Review }\end{array}$ & $\begin{array}{l}\text { December } 2008 \\
\text { Continuous } \\
\text { April, September } 2008 \\
\text { September-October } \\
2008 \\
\text { February-March } 2009 \\
\text { Continuous }\end{array}$ & $\begin{array}{l}\text { March } 2009 \\
\text { June } 2009 \\
\text { March } 2009 \text { (Board) } \\
\text { May } 2009 \text { (Board) } \\
\text { June } 2009 \\
\text { July } 2009\end{array}$ \\
\hline $\begin{array}{l}\text { International } \\
\text { Monetary Fund }\end{array}$ & $\begin{array}{l}\text { Second Review of PSI and } \\
\text { Request for ESF } \\
\text { Third Review of PSI and First } \\
\text { Review under the ESF } \\
\text { Arrangement } \\
\text { Fourth Review of PSI }\end{array}$ & $\begin{array}{l}\text { October/November } \\
2008 \\
\text { March/April } 2009 \\
\text { September/October } \\
2009\end{array}$ & $\begin{array}{l}\text { December } 2008 \text { (Board) } \\
\text { May/June } 2009 \text { (Board) } \\
\text { November/December } 2009\end{array}$ \\
\hline \multicolumn{4}{|c|}{ B. Requests for work program inputs } \\
\hline $\begin{array}{l}\text { Fund request to } \\
\text { Bank (with } \\
\text { summary } \\
\text { justification) }\end{array}$ & $\ldots$ & $\ldots$ & $\cdots$ \\
\hline $\begin{array}{l}\text { Bank request to } \\
\text { Fund (with } \\
\text { summary } \\
\text { justification) }\end{array}$ & $\ldots$ & $\ldots$ & $\ldots$ \\
\hline \multicolumn{4}{|c|}{ C. Agreement on joint products and missions } \\
\hline $\begin{array}{l}\text { Joint products } \\
\text { in next } 12 \\
\text { months }\end{array}$ & $\begin{array}{l}\text { JSAN } \\
\text { DSA }\end{array}$ & $\begin{array}{l}\ldots \\
\text { March/April } 2009\end{array}$ & $\begin{array}{l}\text { December } 2008 \\
\text { May/June } 2009\end{array}$ \\
\hline
\end{tabular}




\section{Senegal: Statistical Issues}

1. Data provision has some shortcomings, but is broadly adequate for surveillance and program monitoring. However, there are weaknesses in data on national accounts, production, and social indicators. The authorities are committed to improving the quality and availability of economic, financial and social indicators, partially relying on technical assistance from the Fund and other international organizations. A Report on the Observance of Standards and Codes was published on the IMF website on December 2, 2002.

2. The country has begun the process of regional harmonization of statistical methodologies within the framework of the West African Economic and Monetary Union (WAEMU). It participates in the General Data Dissemination System (GDDS), and its metadata were posted on the Fund's Dissemination Standards Bulletin Board on September 10, 2001. In September 2006, the authorities expressed their commitment to work toward subscription to the Special Data Dissemination Standard (SDDS) and have appointed a national SDDS coordinator. The November 2008 SDDS assessment mission evaluated dissemination practices against SDDS requirements for coverage, periodicity and timeliness and, in cooperation with the authorities, developed an action plan to address identified gaps.

\section{Real sector statistics}

3. The compilation of the national accounts generally follows the System of National Accounts, 1993. Despite staff's professionalism, the lack of adequate financial resources has constrained efforts to collect and process data. Data sources are deficient in some areas, particularly the informal sector. Owing to financial constraints, surveys of business and households are not conducted regularly. However, efforts are being made to improve data collection procedures, strengthen the coordination among statistical agencies, and reduce delays in data dissemination.

4. The Regional Technical Assistance Center for West Africa (West AFRITAC) has been assisting member countries, including Senegal, with the improvement of their real sector statistics, in particular annual and quarterly national accounts. Progress reported by the advisor includes: i) completion of national accounts for 1980-2004 with 1999 as the base year; ii) dissemination of the 1980-2003 series in hard copy and on the internet; iii) production of accounts by institutional sector (first series covers 2004 institutional sector accounts); and iv) production of national accounts in accordance with the dissemination schedule. The authorities plan to start production of quarterly national accounts in view of the country's intention to subscribe to the SDDS. The recent West AFRITAC missions have assisted with training to support compilation of the quarterly accounts and initiating the compilation of the quarterly accounts for the period 1990-2007. During its most recent mission, the West AFRITAC expert agreed with the authorities on a detailed work program aimed at starting regular dissemination of the quarterly national accounts in March 2010.

\section{Government finance statistics (GFS)}

5. GFS are compiled by the Ministry of Economy and Finance from customs, tax, and treasury directorate sources. Data last reported to STA for electronic redissemination and publication in the 2007 Government Finance Statistics Yearbook were for fiscal year 2001. 
Higher frequency data are not provided for redissemination in IFS, but the ministry compiles and disseminates quarterly government financial operations tables (TOFE) in their own publications. However, deficiencies in the monitoring system for domestic arrears data and other payment delays (including weaknesses in the Treasury computerization system) are being addressed under the PSI program.

6. An AFR team worked with the authorities in February 2004 to improve fiscal reporting in the context of the last PRGF-supported program. The team focused on (i) public accounts that are outside of the direct purview of the treasury; (ii) the treatment of correspondents' accounts in the TOFE; and (iii) ensuring consistency between treasury and banking system information concerning government transactions. The proposed changes are now being implemented. They have improved the presentation of government financial operations and are the first step toward bringing the TOFE more in line with the extended WAEMU TOFE. Other steps will include implementing the WAEMU fiscal directives that are being revised.

7. A regional advisor in GFS has been conducting technical assistance missions aimed at improving the consistency of fiscal reporting and migrating to the methodologies of the Government Finance Statistics Manual 2001. The regional advisor also supported efforts to resume reporting of annual and higher frequency data for publication in International Financial Statistics (IFS) and electronic dissemination of the GFS Yearbook.

\section{Monetary and financial statistics}

8. Preliminary monetary data are compiled by the national agency of the Central Bank of West African States (BCEAO) and officially released (including to the IMF) by BCEAO headquarters. The authorities are now reporting monetary data to STA on a regular basis, with a reduction in the lag from about six months to about three to four months. There has also been an improvement in the timeliness of reporting interest rate and depository corporation data. An area-wide page for the WAEMU zone was introduced in the January 2003 issue of IFS.

9. In 2005, the BCEAO made substantial revisions to the estimates of banknotes in circulation in member states resulting from cross-border banknote movement. These revisions were due to changes in the method to estimate currency in circulation in the WAEMU countries. The revised method, based on updated sorting coefficients ("coefficients de tri”), has been applied retroactively from December 2003.

10. In August 2006, as part of the authorities' continuing efforts to implement the statistical methodology recommended in the Monetary and Financial Statistics Manual, the BCEAO reported to STA test monetary data for June 2006 for all member countries using the Standardized Report Forms (SRF). In response to STA's comments, the BCEAO has recently provided a revised central bank report form (1SR) as well as test data on other depository corporations (2SR) for review by STA. The BCEAO authorities have not yet responded to the feedback provided by STA on the 2SR test data. The completion of the SRFs (1SR for central bank, 2SR for other depository corporations, and 5SR for monetary aggregates) will allow for publication of the data in the IFS Supplement. The publication contains data of 
those countries that regularly compile and report to the Fund monetary data in the SRF format.

\section{External sector statistics}

11. Balance of payments statistics are compiled by the Senegalese national agency of the BCEAO. With STA support over the past few years, several steps have been taken to address certain shortcomings, including: (i) implementation of the Balance of Payments Manual, fifth edition; (ii) modification and simplification of related surveys for companies and banks; (iii) improvement in the computerization of procedures; and (iv) significant strengthening of staff training. Nevertheless, further steps could be taken to enhance the quality and coverage of the balance of payments statistics.

12. Although definitive balance of payments statistics can now be provided with a delay of less than one year, there are some delays in reporting the data to STA. At this time, balance of payment statistics up to the year 2004 and international investment position statistics up to 2006 have been communicated to STA. 
Senegal: Table of Common Indicators Required for Surveillance

(As of December 8, 2008)

\begin{tabular}{|c|c|c|c|c|c|c|c|}
\hline & \multirow[b]{2}{*}{$\begin{array}{l}\text { Date of latest } \\
\text { observation }\end{array}$} & \multirow[b]{2}{*}{ Date received } & \multirow[b]{2}{*}{$\begin{array}{l}\text { Frequency of } \\
\text { data }^{7}\end{array}$} & \multirow[b]{2}{*}{$\begin{array}{l}\text { Frequency of } \\
\text { reporting }\end{array}$} & \multirow[b]{2}{*}{$\begin{array}{l}\text { Frequency of } \\
\text { publication }^{7}\end{array}$} & \multicolumn{2}{|c|}{ Memo Items: } \\
\hline & & & & & & $\begin{array}{c}\text { Data Quality - } \\
\text { Methodological } \\
\text { soundness } \\
\end{array}$ & $\begin{array}{l}\text { Data Quality Accuracy } \\
\text { and reliability }\end{array}$ \\
\hline Exchange Rates & Current & Current & $\mathrm{D}$ & M & M & & \\
\hline $\begin{array}{l}\text { International Reserve Assets and Reserve Liabilities of } \\
\text { the Monetary Authorities } 1\end{array}$ & $9 / 2008$ & $12 / 2008$ & M & M & M & & \\
\hline Reserve/Base Money & $9 / 2008$ & $12 / 2008$ & M & M & M & \multirow{4}{*}{$\mathrm{LO}, \mathrm{LO}, \mathrm{O}, \mathrm{O}$} & \multirow{4}{*}{$\mathrm{LO}, \mathrm{O}, \mathrm{O}, \mathrm{LO}$} \\
\hline Broad Money & $9 / 2008$ & $12 / 2008$ & M & M & M & & \\
\hline Central Bank Balance Sheet & $9 / 2008$ & $12 / 2008$ & M & M & M & & \\
\hline Consolidated Balance Sheet of the Banking System & $9 / 2008$ & $12 / 2008$ & & & & & \\
\hline Interest Rates $^{2}$ & $10 / 2008$ & $11 / 2008$ & M & M & M & & \\
\hline Consumer Price Index & $9 / 2008$ & $10 / 2008$ & M & M & M & $\mathrm{O}, \mathrm{LO}, \mathrm{O}, \mathrm{O}$ & $\mathrm{LO}, \mathrm{O}, \mathrm{O}, \mathrm{NA}$ \\
\hline $\begin{array}{l}\text { Revenue, Expenditure, Balance and Composition of } \\
\text { Financing }{ }^{3} \text { - General Government }\end{array}$ & NA & NA & & & & \multirow[b]{2}{*}{$\mathrm{O}, \mathrm{LNO}, \mathrm{LO}, \mathrm{O}$} & \multirow[b]{2}{*}{$\mathrm{LO}, \mathrm{LO}, \mathrm{O}, \mathrm{LO}$} \\
\hline $\begin{array}{l}\text { Revenue, Expenditure, Balance and Composition of } \\
\text { Financing }{ }^{3} \text { - Central Government }\end{array}$ & Q3/2008 & $11 / 2008$ & Q & Q & $\mathrm{Q}$ & & \\
\hline \multicolumn{8}{|l|}{$\begin{array}{l}\text { Stocks of Central Government and Central Government- } \\
\text { Guaranteed Debt }\end{array}$} \\
\hline External Current Account Balance & 2007 & $05 / 2008$ & A & $\mathrm{A}$ & $\mathrm{A}$ & \multirow[b]{2}{*}{$\mathrm{O}, \mathrm{O}, \mathrm{O}, \mathrm{O}$} & \multirow[b]{2}{*}{$\mathrm{O}, \mathrm{O}, \mathrm{O}, \mathrm{O}$} \\
\hline Exports and Imports of Goods and Services & 2007 & $05 / 2008$ & A & A & A & & \\
\hline GDP/GNP & 2007 & $12 / 2008$ & A & I & $\mathrm{A}$ & LO, LO, LO, LNO & LNO, LNO, LNO, LNO \\
\hline Gross External Debt & 2007 & 05/2008 & Q & I & A & & \\
\hline International Investment Position ${ }^{6 /}$ & 2006 & $9 / 2008$ & A & A & $\mathrm{A}$ & & \\
\hline
\end{tabular}

${ }^{1}$ Includes reserve assets pledged or otherwise encumbered as well as net derivative positions.

${ }^{2}$ Both market-based and officially-determined, including discount rates, money market rates, rates on treasury bills, notes and bonds.

${ }^{3}$ Foreign, domestic bank, and domestic nonbank financing.

${ }^{4}$ The general government consists of the central government (budgetary funds, extra budgetary funds, and social security funds) and state and local governments.

${ }_{6}^{5}$ Including currency and maturity composition.

${ }^{6}$ Includes external gross financial asset and liability positions vis-à-vis nonresidents.

${ }^{7}$ Daily (D); Weekly (W); Monthly (M); Quarterly (Q); Annually (A); Irregular (I); Not Available (NA).

${ }^{8}$ Reflects the assessment provided in the data ROSC published in November 2002 and based on the findings of the mission that took place in September 2001 for the dataset corresponding

to the variable in each row. The assessment indicates whether international standards concerning (respectively) concepts and definitions, scope, classification/sectorization, and basis for

recording are fully observed (O), largely observed (LO), largely not observed (LNO), not observed (NO), or not available (NA).

${ }^{9}$ Same as footnote 7 , except referring to international standards concerning (respectively) source data, statistical techniques, assessment and validation of source data, and revision studies. 


\section{Statement by the IMF Staff Representative on Senegal \\ December 19, 2008}

1. This statement summarizes developments in Senegal since the issuance of the staff report. The information does not change the thrust of the staff appraisal.

2. Inflation sharply dropped in November 2008. End-of-period inflation fell to 4.2 percent in November, after 7.5 percent in October, in part on account of lower food prices related to the good harvests. This bodes well for the projected average inflation projections for 2008 and 2009 underlying the macroeconomic framework.

3. The remaining prior action on the adoption of the $\mathbf{2 0 0 9}$ budget in line with the macroeconomic framework was met. This measure completes a series of upfront measures that are intended to remove spending pressures, provide relief to the government's cash-flow situation, and illustrate the government's commitment to give utmost priority to the expeditious settlement of the unpaid bills. The other measures already taken include: (i) the adoption of the 2008 supplementary budget in November 2008, bringing the expenditure cuts to close to $3 \frac{1}{2}$ percent of GDP relative to the initial budget (prior action); (ii) the elimination of all food and energy subsidies, except for the butane gas subsidy which will be eliminated by mid-2009; and (iii) the discontinuation of Treasury advances, one of the root causes of the budgetary slippages (prior action).

4. Over the last two weeks, the authorities have raised domestic financing in line with the macroeconomic framework. Among other things, they sold their freely tradable shares in the telecommunications company SONATEL at the Abidjan stock exchange and issued T-bills in the WAEMU regional market. However, the latest SIGFIP table of December 12, 2008 indicates that the stock of unpaid bills has not yet been affected by the financing operations; the authorities reiterated to staff that the stock's reduction by more than half by end-2008 remains nonetheless feasible.

5. The government finalized the terms of reference for the technical audit of extrabudgetary spending. The terms of reference fully reflect the staff's comments and are consistent with MEFP paragraph 26. They specify that the exercise is to be conducted by a reputable international audit company with experience in public sector accounting and should be completed by end-March 2009 .

6. The authorities already met the end-January 2009 structural benchmark on the implementation decree for the microfinance law. This measure will enhance the supervision of this rapidly growing sector (which accounts for one-tenth of credit to the economy), introduce stricter licensing and accounting standards, and likely lead to a welcome consolidation in the sector. It follows an earlier benchmark on the microfinance law itself. 
7. The following additional information on the balance-of-payments (BOP) shock is provided as further justification for the ESF request. The first table below illustrates additional indicators on the 2008 BOP impact, going beyond the methodology preferred by the staff as a measure of the exogenous shock for ESF purposes and included in the staff report. This methodology based on the assumption of constant 2007 trade volumes measured the shock at 5.2 percent of GDP. Using trade volumes for 2008 (which reflect the full income (cash) effect — including potentially forced adjustment — and substitution effect) or for the average of 2007 and 2008 (which allow for some income and substitution effects), the BOP impact still amounts to 4.4-4.8 percent of GDP. Overall, Senegal is projected to spend 2.4 percent of GDP more for food and fuel products on a net basis in 2008 than in 2007, with rice accounting for three-fourths of this increase. ${ }^{1}$ Although rice represents a key staple for Senegalese poor households, the volume of rice imports declined by more than 10 percent in 2008. This likely represents both a substitution and an income effect, likely reflecting some forced adjustment, which occurred in spite of the temporary government subsidy on rice.

\section{The detailed calculations for 2009 (second table below) show an abating food} and fuel price shock, implying that it may be temporary in nature. The BOP impact ranges within 1.4-4.9 percent of GDP, and Senegal would spend broadly the same amount relative to GDP for food and fuel on a net basis as in 2007. Given the temporary nature of the shock, the financing under the ESF appears to be the appropriate response, as a further adjustment to the shock would have severely impacted the food supply to the poor and the energy supply to the economy. The ESF would allow Senegal's international reserves to recover and average $3 \frac{1}{2}$ months over the medium term, still below the end-2007 level.

\footnotetext{
${ }^{1}$ As domestic production accounts for only one-tenth of total rice consumption, the surge in the rice price severely affected Senegal's BOP in 2008. The WEO's rice price index rose from 100 in 2005 to a peak of 331 in mid-2008 and is now at 196.
} 
Senegal. Food and Fuel Price Impact, 2008

\begin{tabular}{|c|c|c|c|c|c|}
\hline \multirow[b]{3}{*}{ Net food and fuel impact } & $\begin{array}{c}\text { At } 2007 \\
\text { volumes 1/ }\end{array}$ & $\begin{array}{c}\text { At average } \\
2007-08 \\
\text { volumes 1/ }\end{array}$ & $\begin{array}{c}\text { At } 2008 \\
\text { volumes 1/ }\end{array}$ & \multirow[t]{2}{*}{ Actual change 2/ } & \multirow{2}{*}{$\begin{array}{c}\begin{array}{c}\text { Volume change } \\
(2008 \text { over } 2007)\end{array} \\
\text { Percent }\end{array}$} \\
\hline & \multicolumn{3}{|c|}{ Percent of 2008 GDP } & & \\
\hline & -5.2 & -4.8 & -4.4 & -2.4 & $\ldots$ \\
\hline Food & -2.8 & -2.6 & -2.5 & -2.0 & $\ldots$ \\
\hline Exports & 0.1 & 0.0 & 0.0 & 0.2 & 6.4 \\
\hline Groundnuts & 0.1 & 0.1 & 0.1 & 0.1 & -9.4 \\
\hline Fish & -0.1 & -0.1 & -0.1 & 0.2 & 9.4 \\
\hline Imports & 2.8 & 2.7 & 2.5 & 2.2 & -6.3 \\
\hline Rice & 2.4 & 2.2 & 2.1 & 1.9 & -10.6 \\
\hline Wheat & 0.3 & 0.3 & 0.3 & 0.3 & -5.4 \\
\hline Other & 0.1 & 0.1 & 0.1 & 0.1 & -5.5 \\
\hline Fuel & -2.5 & -2.2 & -1.9 & -0.4 & $\ldots$ \\
\hline Exports & 1.0 & 1.0 & 1.0 & 1.0 & 2.2 \\
\hline Imports & 3.4 & 3.1 & 2.9 & 1.4 & -16.8 \\
\hline
\end{tabular}

Sources: Staff estimates.

1/ Reflects price change between 2007 and 2008 (from latest WEO), using volumes that are kept constant as indicated. Relative to projected 2008 GDP (CFAF 5,993 billion).

2/ Change in CFAF value, relative to projected 2008 GDP.

Senegal. Food and Fuel Price Impact, 2009

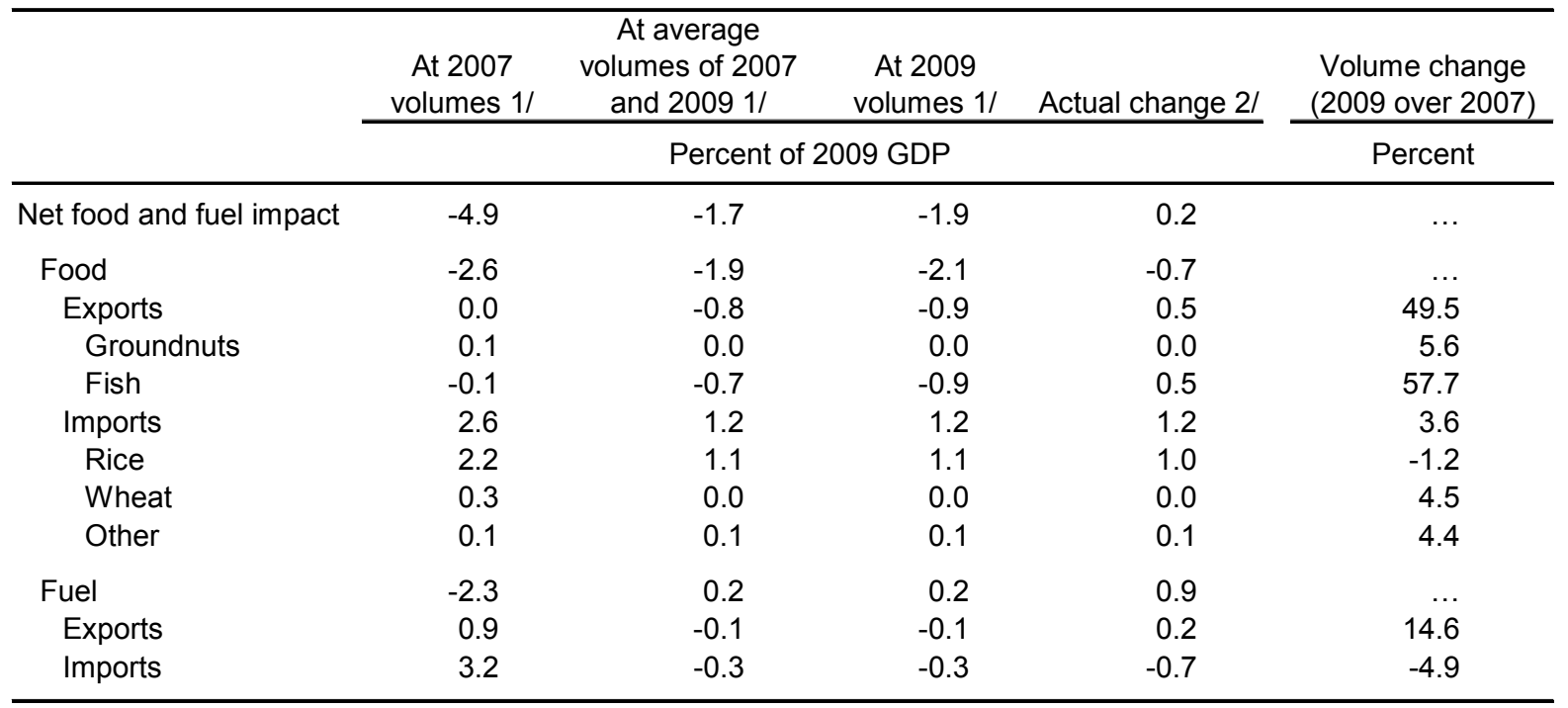

Sources: Staff estimates.

1/ Reflects price change between 2007 and 2009 (from latest WEO), using volumes that are kept constant as indicated. Relative to projected 2009 GDP (CFAF 6,450 billion).

2/ Change in CFAF value, relative to projected 2009 GDP. 
Press Release No. 08/334

International Monetary Fund

FOR IMMEDIATE RELEASE

Washington, D.C. 20431 USA

December 19, 2008

\section{IMF Approves US\$75.6 Million Exogenous Shocks Facility Arrangement for Senegal and Completes Second Review under the Policy Support Instrument}

The Executive Board of the International Monetary Fund (IMF) today approved a one-year SDR 48.54 million (US\$75.6 million) arrangement under the Exogenous Shocks Facility (ESF) to help finance the balance-of-payments impact of higher food and energy prices. The approval enables Senegal to draw an amount of SDR 24.27 million (about US\$37.8 million) from the IMF immediately, and an equal amount upon completion of the first review under the ESF arrangement.

The Board also completed the second review under the three-year Policy Support Instrument (PSI) for Senegal and granted three waivers in view of the strong corrective measures the government is taking to address underlying weaknesses. The waivers were granted for the non-observance of three quantitative assessment criteria, on domestic arrears, the budgetary float (the difference between government payments due and paid), and the contracting or guaranteeing of new nonconcessional external debt by the government.

The PSI for Senegal was approved on November 2, 2007 (see Press Release No. 07/246) and is aimed at consolidating macroeconomic stability, increasing the country's growth potential, and reducing poverty. The program focuses on maintaining a sound fiscal policy stance and enhancing fiscal governance and transparency. It also includes measures to develop the private sector and increase the financial sector's contribution to growth.

Following the Executive Board's discussion on Senegal, Mr. Murilo Portugal, Deputy Managing Director and Chairman, stated:

"The Senegalese economy is facing a difficult period. Economic growth has slowed significantly, as a result of government payment delays to the private sector and the effect on consumption of high food and energy prices. International price developments have kept inflation high and exerted upward pressure on the external current account deficit. Risks to the economic outlook have been compounded by the difficult international environment. 
"Under these challenging circumstances, the government has rightly emphasized the need to eliminate expeditiously the large stock of unpaid bills by the first half of 2009. The fiscal deficits in 2008 and 2009 have been reduced, and untargeted food and energy subsidies largely eliminated to help unwind budgetary pressures and create financial room for settling payment delays. The government will need to strictly adhere to its 2009 budget, and ensure fiscal transparency.

"The government has reaffirmed its intention to pursue a prudent fiscal policy over the medium term while safeguarding priority spending. This should help ensure macroeconomic stability and keep Senegal's debt sustainable. Equally important will be for the government to assess carefully the available financing for its budget, as liquidity in the domestic financial market is limited and borrowing prospects internationally are uncertain. To help debt sustainability, the government should use the proceeds from any asset sales to first repay loans carrying the highest interest rates.

"To help prevent a recurrence of the payment delays, the government should rapidly and thoroughly follow through on its commitments to overhaul its public financial management systems. The reforms are fundamental to restoring the integrity of the budget system, by improving fiscal accounting and monitoring, strengthening budget formulation and execution, and discontinuing practices inconsistent with Senegal's overall satisfactory budgetary, legal, and regulatory framework and administrative capacity. The government should also take appropriate and immediate decisions following completion of the independent technical audit of past extrabudgetary spending to rapidly and fully normalize financial relations with the private sector.

"Progress with structural reforms should continue, with the aim of strengthening private sector activity and bolstering the financial sector. Planned actions include revising labor market regulations, simplifying tax procedures, and improving access to credit.

"Inaccurate information has been provided to the Fund on the fiscal deficit and budgetary float. In light of the substantial corrective measures that the authorities have taken and the improved fiscal monitoring systems they are committed to implementing, the Board decided to maintain a positive assessment of Senegal's program performance under the PSI and of its potential for continued positive performance going forward," Mr. Portugal said. 


\section{Statement by Laurean Rutayisire, Executive Director for Senegal December 19, 2008}

1. Last June, at the time of the first review of Senegal's PSI-supported program, staff drew the Board's attention on a number of challenges facing the country authorities on the fiscal front, including delays in payments to the private sector and potential occurrence of extra budgetary expenditures. Mindful of the need to promptly address such developments, the authorities wasted no time taking necessary steps to reverse them, consistent with Fund advice. Their Letter of Intent (LOI) and the staff report submitted for Board consideration today describe in a detailed manner the numerous reform measures which the authorities have since undertaken in close consultation with staff. Going forward, the authorities are confident that implementation of additional measures envisaged in the program, including those based on the recommendations made by the recent Fund TA mission, will help cope with these challenges and avoid recurrence of similar developments in the future. In particular, they expect that implementation of their action plan will lead to the settlement of payment delays during the first half of 2009 while improvements in the budgetary framework will no longer leave room for extra budgetary spending.

\section{Recent Policy Challenges and Achievements}

2. As acknowledged by the 2006 Ex-Post Assessment of Fund's longer-term program engagement in Senegal, the authorities have traditionally maintained a prudent fiscal policy under Fund-supported programs. And it is not their intention to make an exception in the context of the current PSI arrangement. However, Senegal went through a series of developments over the past few years which strained its capacities and tested its budgetary framework. Like many other members, the country was severely hit by the food and energy crisis which shook the world economy until recently. The steady increases in energy and food prices fueled inflationary pressures and maintained consumer inflation at high levels. Guided by the desire to ease social tension and spare vulnerable households from additional hardship, the authorities' initial policy response included the provision of food and energy subsidies. At the same time, the authorities were to proceed with the implementation of a much-needed infrastructure development program, as they prepared to host the OIC Summit and ambitioned to foster economic activity and create jobs. Moreover, the protracted disruptions in the operations of Les Industries Chimiques du Sénégal (ICS), one of Senegal's largest exporting companies, delayed the full recovery of its exports. The revenue losses and resource needs generated by the food and energy subsidies, temporary tax and customs duty suspensions, disruption in ICS operations, and infrastructure investment contributed to exacerbating budgetary pressures and further delaying the settlement of payments to the private sector.

\section{Eliminating Payment Delays and Auditing Extra budgetary Expenditures}

3. The authorities attach high priority to the elimination of payments delays, as demonstrated by the drastic measures that have been taken as part of the action plan they recently adopted to that resolve. In particular, total spending cuts made by the authorities 
amounted about $3 \frac{1}{2}$ of GDP last month, as the Parliament approved the 2008 supplementary budget. In addition to facilitating the elimination of outstanding bills, this fiscal adjustment helped contain the fiscal deficit which is estimated to be about 3.3 percent of GDP in 2008, amid the current difficult circumstances. On top of the expenditure reductions, other steps were taken by the authorities as part of their action plan to settle payment delays. Among these is the elimination of virtually all food subsidies granted at the onset of the recent food and oil crisis. Specifically, rice subsidies were removed and the temporary duty and tax exemptions on other foodstuffs were rescinded; so was the specific tax on vegetable oil, consistent with Fund advice. Along with the adoption of a new electricity tariff structure, tariffs were increased. In addition, it is the intention of the authorities to eliminate butane gas subsidies next year.

4. With regard to extra budgetary expenditures, the authorities plan to commission an external independent audit to clarify the genesis of these expenditures, as the financial audit inspectorate of the Ministry of Economy and Finance has completed its audit and estimates of extra budgetary expenditures. Such spending will be regularized and cleared only upon their certification by the audit. Two supplementary budgets planned towards the midst and end of next year will help regularize extra budgetary expenditures which will be confirmed by the independent audit.

\section{Advancing the Structural Reform Agenda}

5. The authorities continue to maintain a perfect record of implementation of structural conditionality set forth under the PSI. As was the case for the first PSI review, all structural reform measures agreed for the second review were implemented-albeit with slight delay in implementing the structural benchmark on the elaboration of a program of issuance of government securities. In addition to the abovementioned structural measures, several other steps were taken by the authorities since the first PSI review. In line with the key PSI objectives, decisive actions were executed with the aim at improving fiscal governance and transparency and strengthening public financial and debt management, and . Most notably these include, but are not limited to, compliance with the ceiling on the share of public sector contracts signed by single tender, publication of the list of contracts awarded, strengthening of tax administration, mitigation of a potential adverse impact of the planned special economic zone on tax revenues, improvements in public investment planning and evaluation, and elaboration of a two-year program of issuance of government securities.

\section{Implementing Quantitative Conditionality}

6. The authorities have recently made some noticeable achievements on the quantitative front. In particular, they were able to meet end-June and end-September 2008 assessment criteria related to the basic fiscal deficit and external payment arrears as well as the indicative target on the share of the value of public sector contracts signed by a single tender. However, implementation of other elements of quantitative conditionality proved more challenging. Abovementioned budgetary pressures led to the nonobservance of 
quantitative assessment criteria related to the budgetary float and domestic arrears and gave rise to extra budgetary spending by some public entities. In addition, successful implementation of the authorities' action plan to eliminate payment delays will necessitate recourse to nonconcessional borrowing. As noted by staff, contracting such nonconcessional borrowing became unavoidable for the authorities in view of the pressing need to revive economic activity through the clearance of payment delays and the unattractive terms under which borrowing from the regional and global markets would have been contracted. We call on Directors to support the proposal to raise the ceiling on nonconcessional external borrowing so as to accommodate the nonconcessional loan which the authorities intend to contract from France.

7. In response to Management's letter on the misreporting, the authorities acknowledged that fiscal data communicated to the staff underreported the end-December 2007 budgetary float and basic fiscal balance, as they did not account for subsequent changes to the Treasury accounts that resulted from revenues and payment orders for 2007 which occurred between the time of data reporting and the June Board meeting on the first PSI review. They have since taken strong corrective actions which will help avoid the recurrence of similar shortcomings in the future, including the discontinuation of Treasury advances, timely closure of the fiscal year, strengthening of the expenditure tracking system, and other measures aimed at improving the planning, monitoring and execution of the budget.

8. In light of the strong corrective actions described above, the authorities request waivers for the nonobservance of assessment criteria on domestic arrears and budgetary float.

\section{Policy and Reform Agenda under the PSI}

9. Eager to preserve macroeconomic stability and fiscal sustainability, the authorities are determined to build on aforementioned achievements and pursue reforms needed to address recent shortcomings in an effective and lasting manner and make progress toward the PSI objectives.

\section{Implementing Prudent Fiscal Policies and Strengthening Fiscal Governance and Transparency}

10. They intend to keep the overall fiscal deficit in check through the implementation of prudent policies. In line with the policy intentions expressed by the authorities during program discussions, the Parliament recently adopted a budget law for 2009 which is consistent with the objective of keeping the fiscal deficit below 3 percent of GDP that year. Going forward, the authorities will continue to abide by their commitment to limit the fiscal deficit to 4 percent.

11. A bulk of the fiscal reforms which are underway aims to improve public financial management so as remedy to current weaknesses in the budgetary framework. The planning, execution, and monitoring of the budget will be enhanced through specific actions scheduled to be taken from early in 2009. Budget preparation from 2010 onwards will be facilitated by 
the implementation of the timetable and techniques of budget formulation which will be set by a decree to be adopted by early next year. Monitoring of budgetary execution will gain from the restrictions which will be placed by decree on the amount of expenditure carryovers. It will be also facilitated by the manual which summarizes procedures for reconciling data from budgetary and Treasury accounts. This manual was prepared by the authorities in close consultation with staff and published upon its finalization. Furthermore, the planned audit of budgetary management system, SIGFIP, will be also critical to ascertaining that it remains reliable and continues to guide effectively budgetary management.

12. As part of their endeavors to streamline budgetary procedures and improve transparency, the authorities amended public accounting regulations on November 28, 2008. As a result, recourse to exceptional procedures such as the use of treasury advances is now prohibited. Transparency of budgetary procedures will be also served by the decision to finalize and publish detailed budgetary data by April 30. Other measures which the authorities plan to implement to improve fiscal governance and transparency are noteworthy, including the setup of a clear timetable for submitting Treasury accounts to the audit court and the conduct and publication of an audit of government contracts by the end of the first quarter of 2009.

\section{Pursuing Financial Sector Reform and Coping with the Global Financial Crisis}

13. The authorities intend to press ahead with financial sector reforms which they have been steadily advancing. As noted above, the program of regular issuance of government securities was recently developed, and it is the authorities' intention to implement it in an adequate manner. Following the recent enactment of the new microfinance law, the authorities have recently adopted the inherent implementation decree, which runs toward the authorities' broader objectives of improving the supervision of the microfinance institutions and easing credit access for SMEs.

14. To date, the global financial crisis has not had a significant impact on Senegal's financial system. Nevertheless, the authorities will remain vigilant and stand ready to take necessary actions, should the crisis threaten to undermine the health of the system. Along with their regional counterparts, the authorities have adopted during a high-level meeting held early this month in Abidjan a proactive approach to mitigate any potential adverse effects of the crisis.

\section{Promoting Private Sector Development and Strengthening Debt Management}

15. Consistent with previously stated policy intentions, the authorities undertook several reforms aimed at improving the business environment, earning Senegal a position among the five top performers from the World Bank's Doing Business 2009, including the top reformer globally in easing trade. Key measures that were recently implemented include the setup of a fully operational one-stop shop for business creation, streamlining of import and export procedures, introduction of a single window for customs clearance, and 
significant improvements in infrastructure. Going forward, the authorities intend to pursue reforms aimed at laying the ground for a private sector driven economy, as set forth in their Accelerated Growth Strategy. Planned actions include, among others, the revision of labor market regulations, the simplification of tax procedures, and development of an action plan for improved credit access.

16. Debt management will be a key area on which the authorities plan to put increased focus as they press ahead with their reform agenda and public investment program. In this regard, the authorities are exploring avenues for strengthening debt management capacity and better managing fiscal risks and guarantees. In particular, they have sought technical expertise from the US Treasury in this area and have invited some international rating agencies to assess the level of country risk with a view to identifying the steps that need to be taken to reduce it.

\section{ESF Request}

17. As a major oil and food importer, Senegal has experienced a major BOP shock as a result of the recent oil and food price hikes. These hikes contributed to the double-digit annual percentage increases in the country's import bill over the past couple of years. In order to cope with the adverse impact of the oil and food price shocks, the authorities request Fund assistance under a one-year ESF high-access component. Coupled with Fund financial assistance, the recent reversal of various revenue-reducing and costly measures such as food subsidies and temporary tax suspension on some food and energy products will be instrumental in mitigating the adverse impact of the oil and price shocks and thus enhancing the prospects for improving the fiscal position.

\section{Conclusion}

18. The authorities look forward to the continuation of Senegal's program relation with the Fund under the PSI and the ESF. As emphasized in the LOI, the PSI remains for the authorities the appropriate vehicle to maintain a close policy dialogue with the IMF, signal commitment to sound policies to the international community, and move the structural reform agenda forward. As discussed below, recent actions implemented by the authorities signal their strong commitment to the program while forward-looking measures which they envisage to carry out provide key assurances that the abovementioned shortcomings will be promptly addressed and their reappearance avoided. In their journey under the PSI, and hopefully the ESF, the authorities will continue to value Fund advice.

19. In light of the above, I call on Directors to support the authorities' request for a one-year arrangement under the ESF, waivers and modification of assessment criteria, and the completion of the second PSI review. 